Approved for public release; distribution is unlimited.

\title{
Analysis of Sediment, Water, and Biological Samples From the Bay Farm Borrow Area, San Francisco Bay, California
}
R. M. Thom
L. F. Lefkovitz
Battelle/Marine Sciences Laboratory
Sequim, Washington

August 1991

Prepared for the

U.S. Army Corps of Engineers,

San Francisco District

under a Related Services Agreement

with the U.S. Department of Energy

Contract DE-AC06-76RLO 1830

Pacific Northwest Laboratory

Operated for the U.S. Department of Energy

by Battelle Memorial Institute 


\title{
DISCLAIMER
}

This report was prepared as an account of work sponsored by an agency of the United States Government. Neither the United States Government nor any agency thereof, nor Battelle Memorial Institute, nor any of their employees, makes any warranty, expressed or implied, or assumes any legal liability or responsibility for the accuracy, completeness, or usefulness of any information, apparatus, product, or process disclosed, or represents that its use would not infringe privately owned rights. Reference herein to any specific commercial product, process, or service by trade name, trademark, manufacturer, or otherwise does not necessarily constitute or imply its endorsement, recommendation, or favoring by the United States Government or any agency thereof, or Battelle Memorial Institute. The views and opinions of authors expressed herein do not necessarily state or reflect those of the United States Government or any agency thereof.

\author{
PACIFIC NORTHWEST LABORATORY \\ operated by \\ BATTELLE MEMORIAL INSTITUTE \\ for the \\ UNITED STATES DEPARTMENT OF ENERGY \\ under Contract DE-ACO6-76RLO 1830
}

Printed in the United States of America

Available to DOE and DOE contractors from the

Office of Scientific and Technical Information, P.O. Box 62, Oak Ridge, TN 37831;

prices available from (615) 576-8401. FIS 626-8401.

Available to the public from the National Tecbnical Information Service,

U.S. Department of Commerce, 5235 Port Royal Rd., Springfield, VA 22161. 
PNL -7773

UC -510

ANALYSIS OF SEDIMENT, WATER, AND BIOLOGICAL

SAMPLES FROM THE BAY FARM BORROW AREA,

SAN FRANCISCO BAY, CALIFORNIA

R.M. Thom

L.F. Lefkovitz

Battelle/Marine Sciences Laboratory

Sequim, Washington

August 1991

Prepared for the

U.S. Army Corps of Engineers, San Francisco District under a Related Services Agreement with the U.S. Department of Energy Contract DE-AC06-76RLO 1830

Pacific Northwest Laboratory

Richland, Washington 99352 


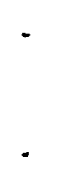




\section{SUMMARY}

The Bay Farm Borrow Area (BFBA) of San Francisco Bay, California, is under consideration as a dredged-material disposal site by the U.S. Army Corps of Engineers (USACE). As part of the analysis of the site, information is required on the quality of benthic biota, sediment, and water in the BFBA. The objective of this report was to provide data on infauna communities, sediment, and water chemistry from samples collected from the BFBA. The samples were collected, and the data will be analyzed by Science Applications International (SAIC).

A total of four samples for sediment chemistry, four samples for water chemistry, and 7 samples for infauna communities were analyzed by the Battelle/Marine Sciences Laboratory (MSL). Water analyses included tests for dissolved organic carbon, total suspended solids, four metals, butyltins, polychlorinated biphenyls (PCBs), chlorinated pesticides, polynuclear aromatic hydrocarbons (PAHs), four phenols, and total phenol. Sediment samples were analyzed for percent solids, total organic carbon, total oil and grease, total petroleum hydrocarbons, grain size, 10 metals, butyltins, PCBs, chlorinated pesticides, PAHs, four phenols, and total phenol. Analyses followed established U.S. Environmental Protection Agency (EPA) procedures where applicable. References were given for those methods not documented by EPA. The animals retained on a $0.5-\mathrm{mm}$ mesh screen from the infauna samples were identified by species and enumerated.

The data, along with controls and spike recovery analyses, are presented in tables, and the results are discussed in the text. The quality assurance/quality control criteria were met for the analyses as were the detection limits specified by the sponsor. Infauna data showed the $4-6$ replicate samples contained virtually all of the species from a station. Hence, seven samples from a station sampled well the number of species at the stations. 


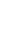




\section{CONTENTS}

SUMMARY i i i

1.0 INTRODUCTION . . . . . . . . . . . . . . . . . . . 1.1

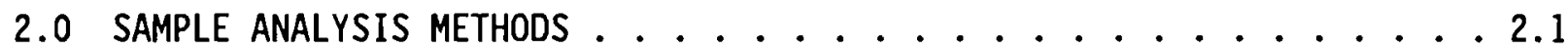

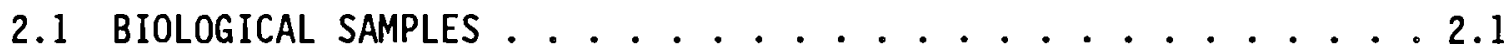

2.1.1 Infauna Methods . . . . . . . . . . . . . 2.1

2.2 CHEMISTRY SAMPLES . . . . . . . . . . . . . 2.8

2.2.1 Sediment Chemistry Methods . . . . . . . . . . 2.8

2.2.2 Water Chemistry Methods . . . . . . . . . . . 2.14

3.0 RESULTS AND QUALITY CONTROL . . . . . . . . . . . . . . . . 3.1

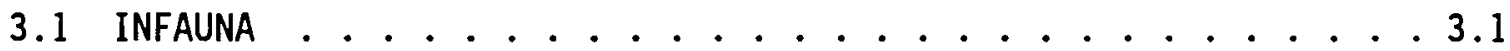

3.2 SEDIMENT CHEMISTRY . . . . . . . . . . . . . . . . 3.1

3.2.1 Total Organic Carbon, $0 i 1$ and Grease, and Total

Petroleum Hydrocarbons . . . . . . . . . . . 3.7

3.2.2 Grain Size . . . . . . . . . . . . . . 3.7

3.2.3 Metals .................... 3.7

3.2.4 Butyltins.................. 3.13

3.2.5 Total Phenols, Phenol, and Substituted Phenols . . . 3.13

3.2.6 Polynuclear Aromatic Hydrocarbons . . . . . . . . 3.17

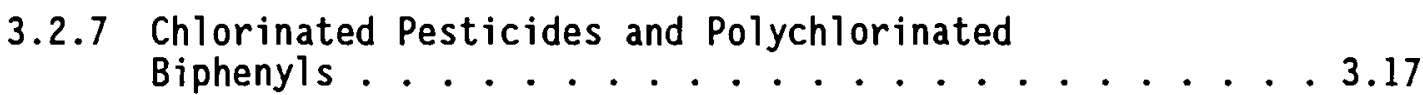

3.3 WATER CHEMISTRY . . . . . . . . . . . . . . . 3.24

3.3.1 Total Suspended Solids, Dissolved Organinc Carbon, $0 i 1$ and Grease, Total Petroleum Hydrocarbons . . 3.24

3.3.2 Metals ..................... 3.24

3.3.3 Butyltins..................... 3.27

3.3.4 Total Phenols, Phenols, and Substituted Phenols . . . 3.27 


\section{CONTENTS (contd)}

3.3.5 Polynuclear Aromatic Hydrocarbons . . . . . . 3.30

3.3.6 Chlorinated Pesticides and Polychlorinated

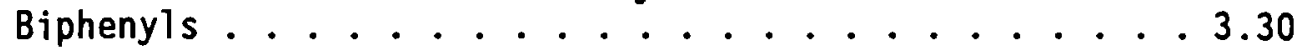

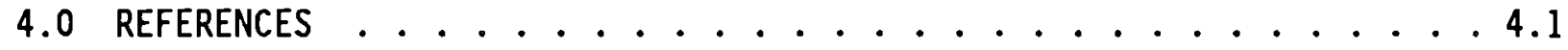

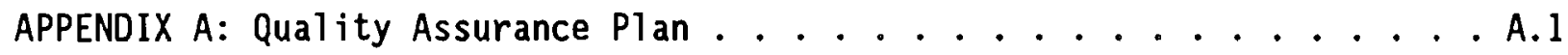

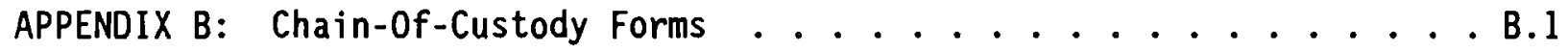




\section{FIGURES}

3.1 Cumulative Number of Infaunal Annelid Taxa Versus Number of Replicate Samples From Each of the Four Stations . . . . . . 3.6

3.2 Plots of Grain Size Fractions in Sample Numbers

E8 Rep. 3, A6 Rep. 3, E4 Rep. 3 . . . . . . . . . . . . 3.11

3.3 Plots of Grain Size Fractions in Sample Number G1l Rep 3. . . . . 3.12

\section{TABLES}

2.1 Samples Delivered to MSL for Processing of Organic Compounds, Trace Metals, Grain Size, Dissolved Organic Carbon (DOC), and Total Suspended Solids (TSS) . . . . . . . . . . . 2.2

2.2 Samples Delivered to MSL for Analysis of Infauna . . . . . . . 2.3

2.3 Species List of Infauna Taxa . . . . . . . . . . . . . 2.5

2.4 Analytical Chemistry Requirements for Bay Farm Sediment and Water Samples ... . . . . . . . . . . . . . 2.9

3.1 Infauna Counts from Station A6 . . . . . . . . . . . 3.2

3.2 Infauna Counts from Station E4 ............... 3.3

3.3 Infauna Counts from Station E8 . . . . . . . . . . . . 3.4

3.4 Infauna Counts from Station G10............... 3.5

3.5 Sediment Conventionals . . . . . . . . . . . . 3.8

3.6 Grain Size . . . . . . . . . . . . . . . . . . 3.9

3.7 Sediment Metals . . . . . . . . . . . . . . . 3.10

3.8 Sediment Butyltins . . . . . . . . . . . . . . . . 3.14

3.9 Sediment Phenols . . . . . . . . . . . . . . . 3.15

3.10 Sediment Polynuclear Aromatic Hydrocarbons . . . . . . . . . 3.18

3.11 Sediment Polycholorinated Biphenyls . . . . . . . . . . . 3.22

3.12 Sediment Cholorinated Pesticides . . . . . . . . . . . 3.23

3.13 Water Conventionals .................. 3.25 


\section{TABLES (contd)}

3.14 Water Metals ...................... 3.26

3.15 Water Butyltins ................... 3.28

3.16 Water Phenols . . . . . . . . . . . . . . . 3.29

3.17 Water Polynuclear Aromatic Hydrocarbons . . . . . . . . . 3.31

3.18 Water Polychlorinated Biphenyls . . . . . . . . . . . . 3.32

3.19 Water Chlorinated Pesticides . . . . . . . . . . . . 3.34 


\subsection{INTRODUCTION}

The Bay Farm Borrow Area (BFBA) of San Francisco Bay, California, is under consideration as a potential dredged-material disposal site as part of the U.S. Army Corps of Engineers' (USACE) Oakland Harbor Navigation Improvement Project. As part of the analysis of this site, information is required on the quality of sediment and water in the BFBA.

To fulfill this requirement, the USACE, San Francisco District, requested the Battelle/Marine Sciences Laboratory (MSL) to analyze sediment and water for organic and trace metals and to determine the number and species of infauna in the samples. Sediment and water samples to be analyzed were collected at designated stations in the BFBA by the USACE, San Francisco District, and Science Applications International Corporation (SAIC) and shipped to the MSL.

Sediment chemical analyses included tests for percent solids, total organic carbon, total oil and grease, total petroleum hydrocarbons, grain size, 10 metals, butyltins, polychlorinated biphenyls, chlorinated pesticides, polynuclear aromatic hydrocarbons, four phenols, and total phenol. Biological analyses included counting the number and determining the infauna species found in samples from each station in the BFBA.

The purpose of this report is to provide the data collected on benthic infauna, sediment, and water samples and document the analytical methods, quality assurance and quality control procedures MSL and participating laboratories used in this process. This report does not describe collection methods.

Science Applications International Corporation will perform a statistical analysis of the MSL-collected data for the USACE and will provide them with conclusions and a discussion of the results. The final results of the analysis will assist the USACE/EPA in determining whether the BFBA site is suitable for open-water disposal pursuant to the requirements of Section 404 of the Clean Water Act. 


\subsection{SAMPLE ANALYSIS METHODS}

Sediment and water samples were collected by SAIC and the USACE, San Francisco District, from the BFBA study area and delivered to the Battelle/ Marine Sciences Laboratory (MSL) at Sequim, Washington. Sample numbers and types are 1 isted in Tables 2.1 and 2.2. The samples were collected within 1 week prior to their delivery to MSL on 19 November 1990 . Water samples were collected on the last sampling day. Infauna core samples were screened through a $0.5-\mathrm{mm}$ mesh sieve in the field and preserved in buffered formalin. All sediment and water samples were held on ice in the dark during transportation to MSL. Four sediment core sections were received for each station. A randomly chosen subset of replicate samples for each station was processed from the stations.

One random sample of the four sediment samples was analyzed for grain size, organic compounds, and trace metals. All water samples except one were analyzed as shown in Table 2.1. A total of seven randomly chosen infauna samples of the 15 replicate samples from each of the four stations were analyzed (Table 2.2).

Archived samples will be retained by MSL until 31 March 1991. The quality assurance/quality control $(Q A / Q C)$ general procedures are presented in Appendix $A$. Specific aspects of $Q A / Q C$ procedures are presented in the appropriate sections below. Copies of the chain-of-custody forms are presented in Appendix B.

\subsection{BIOLOGICAL SAMPLES}

\subsubsection{Infauna Methods}

Infauna samples required further sieving in the laboratory to remove fine sediments to facilitate processing. The samples were placed in $50 \%$ isopropyl alcohol for sorting and identification. All animals retained in the sieved samples were identified to the lowest taxonomic level (usually species) and enumerated (Table 2.3). The bivalve taxa were given preliminary distinctions (e.g., bivalve sp. 1) and were delivered to a bivalve expert for identification and verification. Sorting and identification were performed at Battelle Ocean Sciences Laboratory, Duxbury, Massachusetts. 
TABLE 2.1. Samples Delivered to MSL for Processing of Organic Compounds, Trace Metals, Grain Size, Dissolved Organic Carbon (DOC), and Total

Suspended Solids (TSS)

\begin{tabular}{|c|c|c|c|}
\hline Station No. & Rep. No. & Sample Type & Analyses/Disposition \\
\hline A6 & 1 & sediment & archived \\
\hline A6 & 2 & sediment & archived \\
\hline A6 & 3 & sediment & organics, metals, grain size \\
\hline A6 & 4 & sediment & archived \\
\hline E4 & 1 & sediment & archived \\
\hline E4 & 2 & sediment & archived \\
\hline E4 & 3 & sediment & organics, metals, grain size \\
\hline E4 & 4 & sediment & archived \\
\hline E8 & 1 & sediment & archived \\
\hline E8 & 2 & sediment & archived \\
\hline E8 & 3 & sediment & organics, metals, grain size \\
\hline E8 & 4 & sediment & archived \\
\hline G10 & 1 & sediment & archived \\
\hline G10 & 2 & sediment & archived \\
\hline Glo & 3 & sediment & organics, metals, grain size \\
\hline G10 & 4 & sediment & archived \\
\hline A6 & & water (1) & metals \\
\hline A6 & & water ( $4 \mathrm{~L})$ & organics, DOC, TSS \\
\hline E4 & 1 & water (1L) & metals \\
\hline E4 & 2 & water (1L) & metals (archived) \\
\hline $\begin{array}{l}\text { E4 } \\
\text { F8 }\end{array}$ & & water (4L) & organics, DOC, TSS \\
\hline $\begin{array}{l}\text { E8 } \\
\text { E8 }\end{array}$ & & $\begin{array}{l}\text { water }(1 L) \\
\text { water }(4 L)\end{array}$ & $\begin{array}{l}\text { metals } \\
\text { oraanics nor Tss }\end{array}$ \\
\hline G10 & & water (1L) & $\begin{array}{l}\text { organics, DUL, ISS } \\
\text { metals }\end{array}$ \\
\hline G10 & 1 & water (4L) & organics, DOC, TSS \\
\hline G10 & 2 & water (4L) & $Q A / Q C$ \\
\hline
\end{tabular}


IABLE 2.2. Samples Delivered to MSL for Analysis of Infauna

Station No.

A6

A6

A6

A6

A6

A6

A6

A6

A6

A6

A6

A6

A6

A6

A6

E4

E4

E4

E4

E4

E4

E4

E4

E4

E4

E4

E4

E4

E4

E4

E8

E8

E8
Rep. No. Analyses/Disposition

archived

infauna

infauna

archived

infauna

infauna

archived

infauna

archived

archived

infauna

archived

archived

infauna

archived

archived

infauna

infauna

archived

infauna

infauna

infauna

archived

archived

archived

infauna

infauna

archived

archived

archived

archived

archived

archived 
TABLE 2.2. (contd)

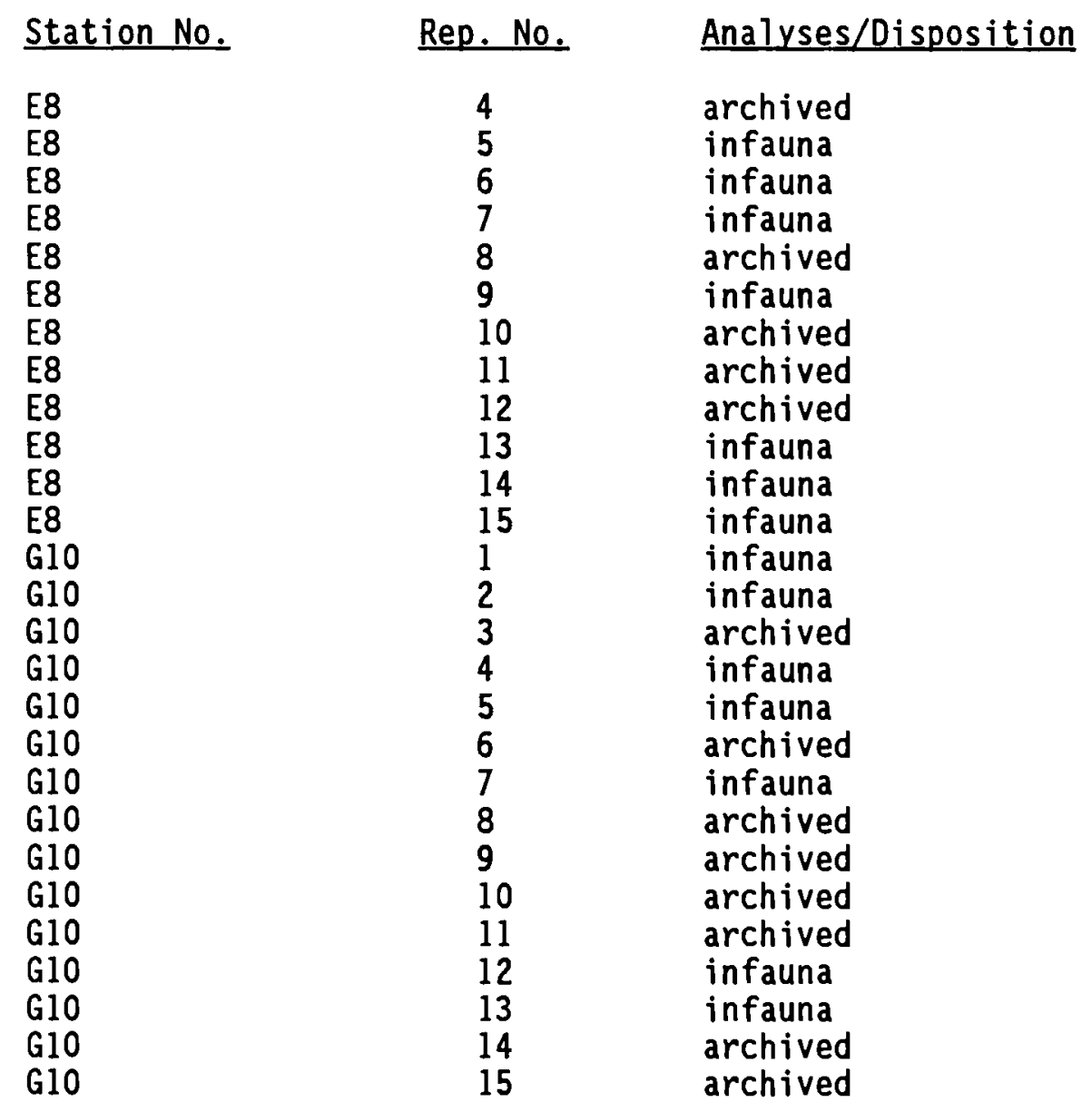


TABLE 2.3. Species List of Infauna Taxa

\section{Crustacea}

Amphipoda

Ampel isca abdita Mi11s, 1964

Corophium al ienense Chapman, 1988

Photis sp. 1, only females or juveniles

amphipod sp. 1, small specimen

Cumacea

cumacea sp. 1, very small specimen

Tanaidacea

Leptochelia cf. savignyi (Kroyer, 1842)

Ostracod

Eusarsiella sp. 1, very similar to Eusarsiella sp. A of SCAMIT

Decapod

Majidae sp. 1, damaged, chelae missing

Natantia, damaged, chelae, most legs missing

Bivalvia

bivalve sp. 1, Potamocorbula amurensis (Schrenck, 1867)

bivalve sp. 2 , most damaged, probably Macoma

bivalve sp. 3

bivalve sp. 4

bivalve sp. 5, smal1 mussels, 1ikely "Musculus" senhousei (Benson,

bivalve sp. 6 1842)

Gemma gemma (Totten, 1834)

Nuculana sp. 1

Annelida

Capitellidae

Barantolla americana Hartman, 1963

Capitella capitata (Fabricius, 1780) species complex

Mediomastus ambiseta (Hartman, 1947)

Mediomastus californiensis Hartman, 1944

Cirratulidae

Cirriformia spirabrancha (Moore, 1904)

Tharyx multifilis Moore, 1909 
TABLE 2.3. (contd)

Cossuridae

Cossura cf. pygodactylata Jones, 1956

Dorvilleidae

Dorvillea rudolphi (delle Chiaje, 1828)

Meiodorvillea sp.

Goni adidae

Glycinde polygnatha Hartman, 1950

Lumbrineridae

Ninoe gemma Moore, 1911

Maldanidae

Asychis elongata (Verrill, 1873)

Nephytidae

Nephtys cornuta fransciscana Clark \& Jones 1955

Opheliidea

Armandia brevis (Moore, 1906)

Orbiniidae

Leitoscoloplos pugettensis (Pettibone, 1957)

Phyllodocidae

Eumida bifoliata (Moore, 1909)

Polynoidae

Harmothoe imbricata (Linnaeus, 1767)

Sabellidae

Euchone limnicola Reish, 1959

Spionidae

Polydora cornuta (Bosc, 1802)

Polydora socialis (Schmarda, 1861)

Pseudopolydora paucibranchiata (Okuda, 1937)

Scolelepis squamata (Muller, 1806)

Spiophanes bombyx (Claparede, 1870)

Syllidae

Autolytus sp.

Exogone lourei Berkeley \& Berkeley, 1938

Sphaerosyllis californiensis Hartman 1966 
TABLE 2.3. (contd)

Syllides longocirrata (Orsted, 1845)

Terebellidae

Polycirrus californicus Moore, 1909

\author{
Nemertea \\ Lineus vegetus Coe, 1931 \\ Micrura alaskensis Coe, 1901
}

Phoronida

Phoronis pallida, (Schneider, 1862) 


\subsection{CHEMISTRY SAMPLES}

The following sections describe the methods used for analysis of sediment and water for the required chemical and physical parameters. A total of four water samples and four sediment samples were analyzed for a variety of parameters.

Sediment analyses included percent solids, total organic carbon (TOC), total 011 and grease (TOG), total petroleum hydrocarbons (TPH), grain size, 10 metals, butyltins, polychlorinated biphenyls (PCBs), chlorinated pesticides, polynuclear aromatic hydrocarbons (PAHs), four phenols, and total phenol.

Water analyses included dissolved organic carbon (DOC), total suspended solids (TSS), four metals, butyltins, polychlorinated biphenyls (PCBS), chlorinated pesticides, polynuclear aromatic hydrocarbons (PAHs), four phenols and total phenol. Analyses followed established U.S. Environmental Protection Agency (EPA) procedures where applicable. References are given for those methods not documented by EPA.

Analyses were performed at five different laboratories. Metals and butyltins were analyzed at MSL. TOC was performed at Global Geochemistry, Canoga, California. PCBs, pesticides, PAHs, substituted phenols in water, oil and grease, and total petroleum hydrocarbons were analyzed at Twin City Testing, St. Paul, Minnesota. Phenols in sediment and total phenol in water were analyzed at Analytical Resources Inc., Seattle, Washington, and grain size analyses were performed at Soil Technologies, Bainbridge Island, Washington.

Table 2.4 lists the analytes and their detection limit goals and quality control requirements. Additional quality control samples included analytical duplicates, matrix spike and matrix spike duplicate analyses, surrogates, method blanks and Standard Reference Materials (SRMs).

\subsubsection{Sediment Chemistry Methods}

\section{Grain Size}

Grain size of sediment samples was determined by a combination of sieve and pipet techniques following the Puget Sound Estuary Program (PSEP) Protocols for Measuring Selected Environmental Variables in Puget Sound (PSEP 
IABLE 2.4. Analytical Chemistry Requirements for Bay Farm Sediment and Water Samples

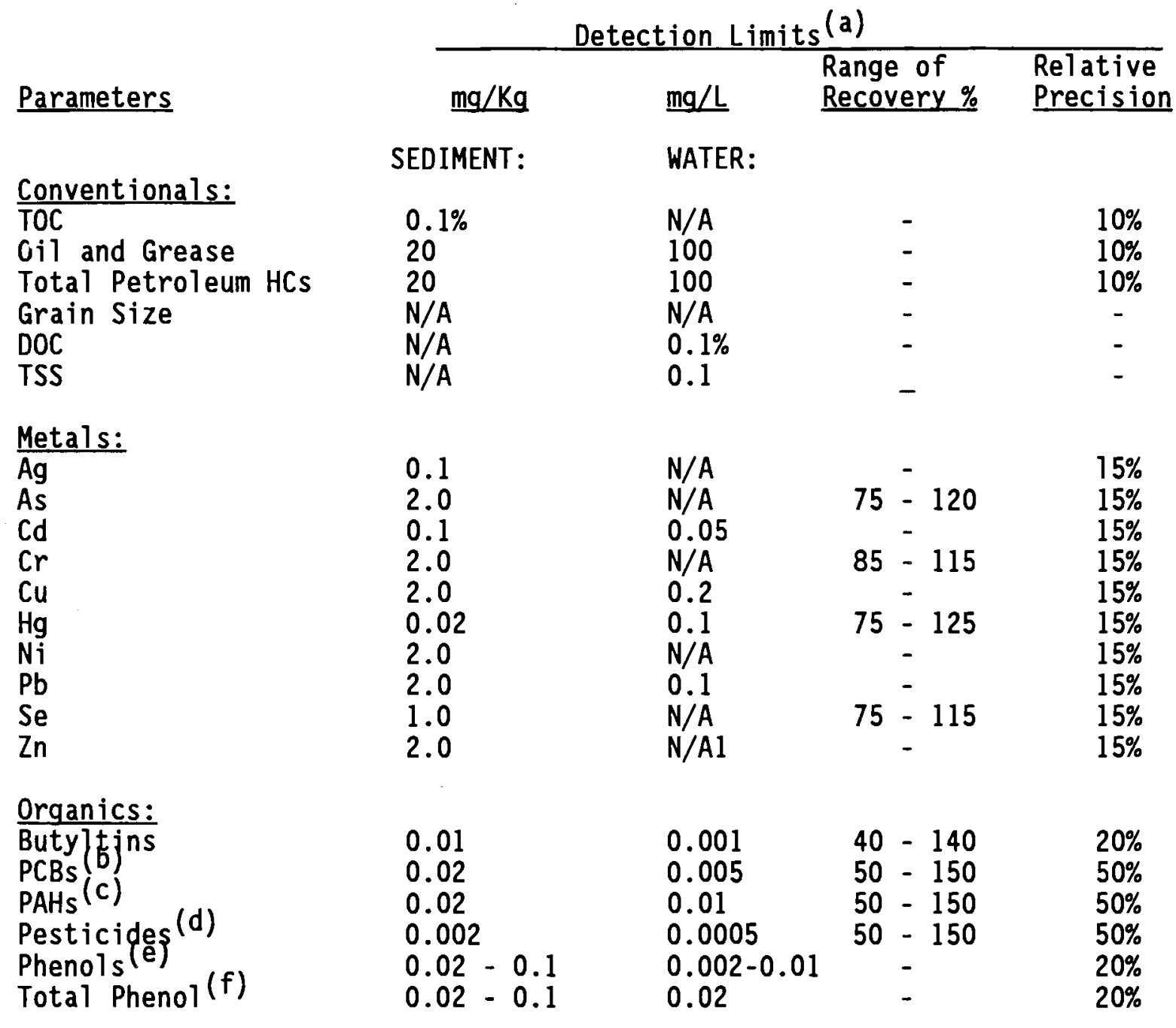

(a) Target detection limits; all efforts were made to reach lowest practical detection limits.

(b) Reported as Aroclor equivalents 1242, 1248, 1254, and 1260 and total PCB, EPA Method 8080.

(c) A11 compounds on EPA Method 610 list. Analyzed using Method 8270 in S.I.M. mode.

(d) A11 compounds on EPA Method 608 list. Analyzed using Method 8080 .

(e) Sediment: Phenols by EPA Method 8040 include Phenol, Pentachlorophenol, 2, 4 dimethylphenol and 2, 4 dichlorophenol. Water: Phenols (same as above) by EPA Method 8270 .

(f) Total Phenol determined colorimetric method $510 \mathrm{C}$ (Standard Methods, 1980).

$\mathrm{N} / \mathrm{A}=$ not applicable.

"-" indicates values are not available. 
1986). These methods are consistent with ASTM D421 (ASTM 1978) and D422 (ASTM 1972) (with the substitution of the No. 100 Sieve for the No. 140 sieve).

Approximately $25 \mathrm{~g}$ of sediment was removed for analysis of total solids while another 10 - to $100-\mathrm{g}$ al iquot was weighed for grain size analysis. To separate the coarser sand and gravel fraction from the silt/clay fraction, sediment was washed with distilled water through a $63.5-\mu \mathrm{m}(4.0 \mathrm{phi})$ sieve into a 1-L graduated cylinder. The coarse fraction was dried, weighed, and shaken through a nest of sieves to yield the required seven coarse subfractions. Any material still passing the final $63.5-\mu \mathrm{m}$ sieve was added to the previous fines in the $1-L$ graduated cylinder.

The silt/clay fraction was then subdivided by a pipet technique based on Stoke's Law of differential settling velocities for different sized particles. The silt/clay fraction was disassociated by a dispersant in distilled water in a 1-L graduated cylinder. At specified time intervals and specified depths below the surface, $20-\mathrm{mL}$ aliquots of suspension were withdrawn from the graduated cylinder, delivered to a preweighed container, and dried to constant weight at $90^{\circ} \pm 2^{\circ} \mathrm{C}$. Quality control measures included duplicate analysis of one sample. Spikes, SRMs, or minimum detection limits do not apply to grain size.

\section{Total Organic Carbon}

The TOC measurement includes the amount of non-volatile, partially volatile, volatile, and particulate organic compounds in a sample. The TOC in sediment was determined by measuring the carbon dioxide released during combustion of the sample (PSEP 1986; SW846 Method 9060, EPA 1986). The sediment sample is dried and ball milled to a fine powder. Before combustion, inorganic carbonate was removed by acidification. Quality control measures included duplicate analysis on $10 \%$ of the samples and analysis of the SRM MESS-I. Results are reported as percent dry weight.

\section{0il and Grease and Petroleum Hydrocarbons}

Total oil and grease includes vegetable oils, animal fats, soaps, waxes, and any other carbon-hydrogen material extractable by the solvent Freon. Total petroleum hydrocarbons are the non-polar mineral oil fraction of total $0 i 1$ and grease. Infra-red spectrophotometry (IR) is used to determine 
concentrations of $0 i 1$ and grease (Method 413.2, EPA 1979) and petroleum hydrocarbons (Method 418.1, EPA 1979). A 20-g al iquot of sample was dried with anhydrous sodium sulfate, then extracted with Freon.

For total oil and grease, sample extracts were scanned from 4000 to 600 $\mathrm{cm}^{-1}$ on an infrared spectrophotometer and the peak height measured at 2930 $\mathrm{cm}^{-1}$. This wavelength represents the $\mathrm{CH}_{2}$ configurations of hydrocarbons and was the standard used to determine oil and grease. For total petroleum hydrocarbons, silica gel was added to the extract to remove the animal- and vegetable-based oils. The extract was then shaken and allowed to settle, and an aliquot was removed and scanned the same way as for oil and grease. The relationship of peak height to the oil concentration was determined by regressing the peak height versus a known concentration of fuel $0 i 1$.

\section{Metals}

Ten metals were measured in BFBA sediments, silver (Ag), arsenic (As), cadmium (Cd), chromium ( $\mathrm{Cr})$, copper $(\mathrm{Cu})$, mercury $(\mathrm{Hg})$, nickel (Ni), lead $(\mathrm{Pb})$, selenium ( $\mathrm{Se})$, and zinc $(\mathrm{Zn})$. Six metals ( $\mathrm{As}, \mathrm{Cr}, \mathrm{Cu}, \mathrm{Ni}, \mathrm{Pb}$ and $\mathrm{Zn}$ ) were measured by energy-diffusive $X$-Ray fluorescence (XRF) following the method of Nielson (1983). Mercury was analyzed by cold-vapor atomic absorption spectroscopy (CVAA) (SW846 Method 7471, EPA 1986; Bloom and Crecelius 1983), while Ag, Cd, and Se were analyzed by Zeeman graphitefurnace atomic absorption spectroscopy (GFAA) (SW846 7000 series, EPA 1986; Bloom and Crecelius 1984).

To prepare sediments for analysis, samples were freeze-dried, then blended in a Spex mixer-mill. Approximately $5 \mathrm{~g}$ of mixed sediment was ground in a ceramic ball mill. The XRF analysis was performed on a $0.5-\mathrm{g}$ aliquot of dried, ground sediment pressed into a pellet of $2 \mathrm{~cm}$ diameter. For GFAA and CVAA analysis, $0.2-g$ aliquots of dried homogenate went through an acid digestion process to separate and isolate the metals from the sediment. Quality control measures for metals analysis included analysis of blanks (not applicable to XRF technique), one set of triplicate analyses and analysis of two SRM samples, National Bureau of Standards (NBS) SRM 1464 and National Research Council of Canada (NRCC) SRM MESS-1. 


\section{Butyltins}

Butyltin compounds were analyzed using gas chromatography with flame photometric detection (GC/FPD) following the methods of Unger et al. (1986). Approximately $10 \mathrm{~g}$ of wet sediment was weighed into a solvent-rinsed jar, dried with anhydrous sodium sulfate, then extracted from sediment with $110 \mathrm{ml}$ methylene chloride and $0.25 \mathrm{~g}$ tropolone. Propyltin was added before extraction as a surrogate compound to assess extraction efficiency. The extract was decanted through silanized glass wool to remove particles, and the container rinsed three times with methylene chloride to ensure that all extracted material was recovered. The mono-, di-, and tributyltin compounds extracted from the sediment were derivatized with $n$-hexyl magnesium bromide to a less volatile, more thermally stable form (nonionic $n$-hexyl derivatives) for analyses.

The extracts were passed through a florisil liquid chromatography column for cleanup, and the butyltins quantified by GC/FPD. Concentrations were reported in $\mu \mathrm{g} / \mathrm{kg}$ dry weight of mono-, di-, and tributyltin species as tin. The recently certified NRCC SRM PACS-1 for butyltins was analyzed along with BFBA sediments.

\section{Total Phenols}

Total phenols were analyzed using Method $510 \mathrm{C}$ (Standard Methods 1980). This method involved steam distillation of the sediments followed by photometric analysis of the distillate after reacting it with 4aminoantipyrine.

One analytical replicate and a matrix spike were analyzed for total phenols.

\section{Substituted Phenols}

Phenol and various substituted phenols were measured in sediment samples from BFBA using EPA SW846 Method 8040. The majority of the phenols were extracted using EPA Method 3550, transferred to hexane, cleaned up using Gel Permeation Chromatography and analyzed using Gas Chromatography/Flame Ionization Detection (GC/FID). To achieve greater sensitivity and lower detection limits, pentachlorophenol and a number of other chlorinated phenols 
were analyzed using GC/ECD after derivatization of the GC/FID extract. This step involved the formation of pentafluorobenzylbromide derivatives followed by a silica gel column chromatography and quantitation using GC/ECD analysis.

Appropriate surrogates were added to the samples prior to extraction. Spiking compounds were added in duplicate to a selected sample and analyzed by both GC/FID and GC/ECD. No SRM is available for phenols in sediment.

\section{Polynuclear Aromatic Hydrocarbons}

The semivolatile organic compounds analyzed in BFBA sediments are the 16 polynuclear aromatic hydrocarbons (PAHs) 1isted in EPA Method 610. These compounds were extracted from sediments following EPA Method 3540 (EPA 1986) using methylene chloride as the extraction solvent. Extracts were dehydrated with sodium sulfate and concentrated to $2 \mathrm{ml}$. A $1-\mathrm{ml}$ portion of this extract was used for PAH analysis by gas chromatography/mass spectroscopy in the Selective Ion Mode (GC/MS SIM) following EPA Method 8270 (EPA 1986).

Three PAH surrogate compounds were added to all samples prior to extraction, and seven PAH compounds were added to a selected sample in duplicate to assess accuracy and precision of the analyses. The NRCC SRM HS5 was also analyzed for PAH compounds, although the levels of PAH compounds in this SRM are relatively high compared to the levels measured in the samples.

\section{Chlorinated Pesticides and Polychlorinated Biphenyls}

Chlorinated pesticides and PCBs in sediments were quantified by GC/ECD following EPA SW846 Method 8080 (EPA 1986). Chlorinated pesticides and PCBs were extracted simultaneously with the PAH compounds using EPA Method 3540 as described above. A $1-m 1$ portion of the methylene chloride extract was solvent exchanged to hexane, and interferences were removed using a $10-\mathrm{g} 7 \%$ deactivated alumina column. Analytical quantification was performed using $G C / E C D$ analysis. Dibutylchlorendate (DBC) was the surrogate compound added to each sample before extraction to assess extraction efficiency.

The matrix spiking solution was also added to the appropriate samples before extraction. Matrix spike/matrix spike duplicate analyses were conducted to assess accuracy and precision of the measurement. One sample was analyzed in duplicate to assess analytical precision. A method blank was 
analyzed along with this set of samples as well. Two SRMs, HS-1 and HS-2, obtained from NRCC were analyzed for PCBs only. Due to interferences present in the SRM material, additional clean-up by Gel Permeation Chromatography (GPC) was performed on these samples.

\subsubsection{Water Chemistry Methods}

All chemical analysis for water were performed on unfiltered water samples with the exception of DOC.

\section{Total Suspended Solids}

Total suspended solids in BFBA water were analyzed using Method 209 D (Standard Methods 1980).

\section{Dissolved Organic Carbon}

DOC was measured using EPA method 360.2 (EPA 1983), which is a modified winkler titration. Prior to titration, samples were filtered through a 0.45 $\mu \mathrm{m}$ nucleopore filter.

\section{0il and Grease}

$0 i 1$ and grease analyses in water were performed as described above for sediment with the exception that a $100-\mathrm{mL}$ aliquot was extracted in a separatory funnel. Results are reported in $\mathrm{mg} / \mathrm{L}$.

\section{Total Petroleum Hydrocarbons}

TPH analysis in water was performed as described above for sediments.

\section{Metals}

Four metals were analyzed in BFBA waters: cadmium (Cd), copper (Cu), mercury $(\mathrm{Hg})$, and lead $(\mathrm{Pb})$. Two of the metals, $\mathrm{Cd}$ and $\mathrm{Pb}$, were analyzed using Inductively Coupled Plasma Emission Mass Spectrometry (ICP/MS). Copper was analyzed by direct injection Graphite Furnace AA (GFAA) (Bloom and Crecelius 1984), and mercury was analyzed by cold-vapor atomic absorption spectroscopy (CVAA) (SW846 Method 7471, EPA 1986; Bloom and Crecelius 1983).

Salt water interferences were reduced during ICP/MS and $A A$ analyses by diluting the samples 2- to 10-fold. Dilution was also used during the analysis of mercury; however, sensitivity was increased by the use of a 
fluorescence detector. Re-analysis of copper is currently in progress. A pre-concentration step will be used to achieve lower detection limits. Data will be presented when available.

Two SRM waters were analyzed along with the samples. These were $1641 \mathrm{~b}$ for $\mathrm{Cd}, \mathrm{Cu}$, and $\mathrm{Pb}$ and $1643 \mathrm{~b}$ for $\mathrm{Hg}$.

\section{Butyltins}

Butyltin compounds in BFBA water were analyzed as described above for sediments. Extraction of the water involved extracting approximately $500 \mathrm{~mL}$ of water with methylene chloride and tropolone in a separatory funnel. No water SRMs are available at this time for analyses. A blank spike was analyzed using Sequim Bay filtered seawater.

\section{Total Phenols}

Total phenols were analyzed as described above for sediments. A method blank, analytical replicate, and matrix spike/matrix spike duplicate were also analyzed. No SRM exists for phenols in water.

\section{Phenols}

Phenols in water from the BFBA were analyzed by EPA SW846 Method 8270 (EPA 1986). This method involves quantitation by GC/MS. Extraction of water followed EPA method 3510 (EPA 1986) and was performed in conjunction with the extraction for semivolatile compounds.

Three acid surrogate compounds were added prior to extraction to assess extraction efficiency. A method blank and a matrix spike and matrix spike duplicate were analyzed to determine both analytical accuracy and precision. No analytical replicate was performed because of insufficient volumes of water.

\section{Polynuclear Aromatic Hydrocarbons}

The semivolatile organic compounds analyzed in BFBA water are the 16 polynuclear aromatic hydrocarbons (PAHs) listed in EPA Method 610. These compounds were analyzed as described above for sediments with the exception that the water was extracted using EPA Method 3510 (EPA 1986) and screened on the Mass Spectrometer (Method 8270) using a full scan (rather than Selective Ion Mode), which results in somewhat higher detection limits. Three base- 
neutral surrogate compounds were added prior to extraction to assess extraction efficiency.

A matrix spike and matrix spike duplicate sample was also analyzed. Spiking compounds for this analyses normally include both acid and base/neutral compounds; however, for this set of samples, the base/neutral spiking compounds were inadvertently left out. Spike recoveries for the acid compounds are reported and appear to indicate good recoveries.

\section{Chlorinated Pesticides and Polychlorinated Biphenyls}

Chlorinated pesticides and PCBs in water were extracted following EPA Method 3510 (EPA 1986). Extracts were cleaned and analyzed as described above for sediments. A method blank was analyzed for both PCBs, and pesticides and a matrix spike and a matrix spike duplicate were analyzed for selected pesticides. No SRM is available for PCBs or pesticides in water. 


\subsection{RESULTS AND QUALITY CONTROL}

\subsection{INFAUNA}

Quality control procedures for the infauna samples involved a continuous monitoring program for al1 laboratory work. Al1 QC records were retained in project files. To ensure that the level of sorting error did not exceed $5 \%$, the sample residues from $10 \%$ of the samples were resorted by another technician. If the resort showed that more than $5 \%$ of the total number of organisms contained in the original sample were found in the resort, the sample was sorted again. When vials were shipped to participating laboratories for identification, individual vials were checked to ensure that they contained only the organisms of the taxon indicated on the label vial. A final comparison of all identified specimen vials against the material recorded on data sheets was made. In cases where the inspection indicated that the number of specimens in the vial did not match the number indicated on the data sheet, specimens were recounted. Verification of voucher-collection specimens by senior-level taxonomists were used to ensure taxonomic accuracy and standardization.

The list of infauna and their densities are shown in Tables $3.1-3.4$. A plot of cumulative number of annelid taxa versus number of replicate samples showed that the number of new taxa encountered in 4-6 replicates probably represented that majority of species present at the station (Figure 3.1).

\subsection{SEDIMENT CHEMISTRY}

The following summarizes the results and quality control for analyses performed on BFBA sediment samples. Data quality objectives for these analyses are presented in Table 2.3.

Overal1, quality control was acceptable. All samples were accompanied by custody forms at all times and samples were held at either $4^{\circ} \mathrm{C}$ or frozen prior to analyses. Holding times were met for all samples with the exception of the re-extraction of sediment samples for PCBs and pesticides because of low surrogate recoveries from the initial extraction. Samples were held frozen, and no deleterious effects are expected. 


\section{TABLE 3.1. Infauna Counts from Station A6}

Taxa
ANNELIDS:
Asychis elongata
Autolytus sp.
Barantolla americana
Cirriformia spi rabrancha
Cossura cf. pygodactylata
Dorvillea rudolphi
Eumida bi fol iata
Exogone lourei
Exogone spp.
Fabriciola berkeleyi
Glycinde polygnatha
Harmothoe imbricata
Leitoscoloplos pugettensis
Mediomastus spp.
Ninoe germa
oligochaeta
Polycirrus californicus
Polydora social is
Pseudopolydora paucibranchiata
Sphaerosyllis californiensis
Spiophanes bombyx
TOTAL ANNELIDS

ARTHROPODS :

Ampel isca abdita

amphipod sp. 1

Corophium alienense

Leptochelia cf. sauvignyi

natantia

Phot is sp. 1

TOTAL ARTHROPODS

MOLLUSCS:

bivalve sp. 2

bivalve sp. 5

bivalve sp. 6

bivalve sp. unidentified

TOTAL MOLLUSCS

OTHER PHYLA

?Micrura alaskensis
Number of individuals $10-\mathrm{cm}$ diameter core sample

\begin{tabular}{|c|c|c|c|c|c|c|c|c|c|}
\hline \multirow[b]{2}{*}{$\underline{2}$} & \multicolumn{6}{|c|}{ Repl icate Number } & \multirow[b]{2}{*}{ Mean } & \multirow[b]{2}{*}{ SDEV } & \multirow[b]{2}{*}{$\underline{\mathrm{CV}}$} \\
\hline & $\underline{3}$ & $\underline{5}$ & $\underline{6}$ & $\underline{8}$ & $\underline{11}$ & $\underline{14}$ & & & \\
\hline 5 & 4 & 2 & 2 & 3 & 6 & 0 & 3.1 & 1.9 & 0.60 \\
\hline 0 & 0 & 11 & 0 & 2 & 1 & 0 & 2.0 & 3.7 & 1.87 \\
\hline 0 & 1 & 0 & 0 & 0 & 0 & 0 & 0.1 & 0.3 & 2.45 \\
\hline 0 & 1 & 0 & 1 & 1 & 1 & 1 & 0.7 & 0.5 & 0.63 \\
\hline 0 & 1 & 0 & 0 & 0 & 0 & 0 & 0.1 & 0.3 & 2.45 \\
\hline 0 & 1 & 0 & 0 & 0 & 2 & 0 & 0.4 & 0.7 & 1.70 \\
\hline 0 & 0 & 0 & 0 & 1 & 0 & 0 & 0.1 & 0.3 & 2.45 \\
\hline 14 & 53 & 168 & 42 & 10 & 67 & 28 & 54.6 & 50.0 & 0.92 \\
\hline 1 & 0 & 8 & 0 & 0 & 3 & 0 & 1.7 & 2.8 & 1.61 \\
\hline 0 & 0 & 0 & 1 & 0 & 0 & 0 & 0.1 & 0.3 & 2.45 \\
\hline 0 & 1 & 1 & 1 & 0 & 0 & 0 & 0.4 & 0.5 & 1.15 \\
\hline 0 & 1 & 3 & 0 & 1 & 0 & 0 & 0.7 & 1.0 & 1.44 \\
\hline 0 & 1 & 1 & 0 & 0 & 2 & 0 & 0.6 & 0.7 & 1.27 \\
\hline 0 & 1 & 0 & 0 & 0 & 0 & 0 & 0.1 & 0.3 & 2.45 \\
\hline 0 & 2 & 0 & 0 & 0 & 0 & 0 & 0.3 & 0.7 & 2.45 \\
\hline 0 & 2 & 10 & 0 & 3 & 3 & 2 & 2.9 & 3.1 & 1.10 \\
\hline 0 & 0 & 0 & 1 & 0 & 0 & 0 & 0.1 & 0.3 & 2.45 \\
\hline 0 & 1 & 0 & 1 & 0 & 2 & 1 & 0.7 & 0.7 & 0.98 \\
\hline 0 & 0 & 0 & 1 & 2 & 1 & 0 & 0.6 & 0.7 & 1.27 \\
\hline 1 & 1 & 0 & 0 & 0 & 1 & 1 & 0.6 & 0.5 & 0.87 \\
\hline 0 & 0 & 0 & 1 & 0 & 0 & 0 & 0.1 & 0.3 & 2.45 \\
\hline 21 & 71 & 204 & 51 & 23 & 89 & 33 & 70.3 & 59.4 & 0.84 \\
\hline
\end{tabular}

$\begin{array}{llllllllll}4 & 21 & 1 & 1 & 5 & 2 & 0 & 4.9 & 6.8 & 1.40 \\ 0 & 0 & 0 & 1 & 0 & 0 & 0 & 0.1 & 0.3 & 2.45 \\ 0 & 5 & 3 & 1 & 0 & 0 & 1 & 1.4 & 1.8 & 1.23 \\ 1 & 0 & 2 & 0 & 0 & 0 & 3 & 0.9 & 1.1 & 1.31 \\ 0 & 0 & 1 & 0 & 0 & 0 & 0 & 0.1 & 0.3 & 2.45 \\ 0 & 1 & 0 & 0 & 0 & 0 & 0 & 0.1 & 0.3 & 2.45 \\ 5 & 27 & 7 & 3 & 5 & 2 & 4 & 7.6 & 8.1 & 1.07\end{array}$

$\begin{array}{llllllllll}0 & 1 & 0 & 0 & 0 & 0 & 0 & 0.1 & 0.3 & 2.45 \\ 1 & 1 & 1 & 1 & 1 & 6 & 3 & 2.0 & 1.8 & 0.89 \\ 0 & 1 & 1 & 0 & 0 & 1 & 0 & 0.4 & 0.5 & 1.15 \\ 0 & 4 & 0 & 0 & 0 & 0 & 0 & 0.6 & 1.4 & 2.45 \\ 1 & 7 & 2 & 1 & 1 & 7 & 3 & 3.1 & 2.5 & 0.81\end{array}$

12.120 .120

$\begin{array}{llllllllll}0 & 0 & 1 & 0 & 0 & 0 & 0 & 0.1 & 0.3 & 2.45\end{array}$ 
TABLE 3.2. Infauna Counts from Station E4

$\underline{T a x a}$

ANNELIDS:

Armandia brevis

Asychis elongata

Barantolla americana

Capitella capitata complex

Euchone limnicola

Exogone lourei

Exogone spp.

Glycinde polygnatha

Harmothoe imbricata

Mediomastus californiensis

Nephtys cornuta franciscana

Polycirrus spp. unidentified

Polydora cornuta

Polydora social is

TOTAL ANNELIDS

ARTHROPODS:

Ampel isca abdita

Corophium alienense

Eusarsiella sp. 1

Leptochelia cf. sauvignyi

Majidae sp. 1

Photis sp. 1

TOTAL ARTHROPODS

MOLLUSCS:

bivalve sp. 2

bivalve sp. 3

bivalve sp. 4

bivalve sp. 5

bivalve sp. 6

bivalve sp. unidentified

Nuculana sp. 1

TOTAL MOLLUSCS

OTHER PHYLA:

?Lineus vegetus ?Micrura alaskensis

Phoronis pallida
Number of individuals $/ 10 \mathrm{~cm}$ diameter core sample

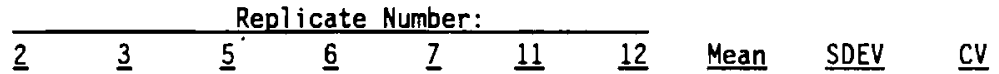

$\begin{array}{llllllllll}0 & 0 & 0 & 0 & 1 & 0 & 0 & 0.1 & 0.3 & 2.45 \\ 3 & 3 & 3 & 3 & 5 & 5 & 6 & 4.0 & 1.2 & 0.30 \\ 1 & 0 & 0 & 0 & 0 & 0 & 0 & 0.1 & 0.3 & 2.45 \\ 0 & 2 & 0 & 0 & 0 & 1 & 0 & 0.4 & 0.7 & 1.70 \\ 0 & 0 & 0 & 0 & 0 & 0 & 1 & 0.1 & 0.3 & 2.45 \\ 8 & 0 & 4 & 3 & 1 & 0 & 3 & 2.7 & 2.6 & 0.96 \\ 0 & 0 & 1 & 0 & 0 & 0 & 0 & 0.1 & 0.3 & 2.45 \\ 1 & 0 & 0 & 0 & 0 & 0 & 0 & 0.1 & 0.3 & 2.45 \\ 1 & 0 & 0 & 0 & 0 & 0 & 0 & 0.1 & 0.3 & 2.45 \\ 1 & 0 & 0 & 0 & 0 & 0 & 0 & 0.1 & 0.3 & 2.45 \\ 1 & 0 & 0 & 0 & 0 & 0 & 0 & 0.1 & 0.3 & 2.45 \\ 1 & 0 & 0 & 0 & 0 & 0 & 0 & 0.1 & 0.3 & 2.45 \\ 1 & 0 & 0 & 0 & 0 & 0 & 0 & 0.1 & 0.3 & 2.45 \\ 0 & 0 & 0 & 0 & 0 & 1 & 1 & 0.3 & 0.5 & 1.58 \\ 18 & 5 & 8 & 6 & 7 & 7 & 11 & 8.9 & 4.1 & 0.47\end{array}$

$\begin{array}{rrrrrrrrrr}100 & 58 & 158 & 53 & 48 & 1 & 136 & 79.1 & 50.8 & 0.64 \\ 1 & 0 & 3 & 0 & 1 & 0 & 1 & 0.9 & 1.0 & 1.15 \\ 0 & 0 & 1 & 0 & 1 & 0 & 1 & 0.4 & 0.5 & 1.15 \\ 0 & 0 & 2 & 0 & 0 & 0 & 2 & 0.6 & 0.9 & 1.58 \\ 0 & 0 & 0 & 0 & 0 & 0 & 1 & 0.1 & 0.3 & 2.45 \\ 1 & 0 & 0 & 0 & 0 & 0 & 0 & 0.1 & 0.3 & 2.45\end{array}$

$\begin{array}{llllllllll}102 & 58 & 164 & 53 & 50 & 1 & 141 & 81.3 & 52.9 & 0.65\end{array}$

$\begin{array}{llllllllll}1 & 2 & 1 & 0 & 2 & 0 & 2 & 1.1 & 0.8 & 0.73 \\ 0 & 1 & 0 & 0 & 0 & 0 & 0 & 0.1 & 0.3 & 2.45 \\ 0 & 2 & 0 & 0 & 0 & 0 & 0 & 0.3 & 0.7 & 2.45 \\ 0 & 0 & 1 & 0 & 0 & 0 & 0 & 0.1 & 0.3 & 2.45 \\ 1 & 0 & 0 & 0 & 0 & 0 & 0 & 0.1 & 0.3 & 2.45 \\ 4 & 0 & 0 & 2 & 2 & 0 & 1 & 1.3 & 1.4 & 1.08 \\ 0 & 0 & 0 & 0 & 0 & 0 & 1 & 0.1 & 0.3 & 2.45 \\ 6 & 5 & 2 & 2 & 4 & 0 & 4 & 3.3 & 1.9 & 0.58\end{array}$

$\begin{array}{rrrrrrrrrr}0 & 0 & 2 & 0 & 0 & 0 & 0 & 0.3 & 0.7 & 2.45 \\ 0 & 0 & 0 & 0 & 0 & 0 & 1 & 0.1 & 0.3 & 2.45 \\ 0 & 33 & 0 & 0 & 0 & 1 & 1 & 5.0 & 11.4 & 2.29\end{array}$




\section{TABLE 3.3. Infauna Counts from Station E8}

$\underline{T a x a}$

ANNELIDS:

Asychis elongata

Capitella capitata complex

Euchone limnicola

Exogone lourei

Glycinde polygnatha

Leitoscoloplos pugettensis

maldanidae spp. juvenile

Mediomastus ambiseta

Mediomastus californiensis

Mediomastus spp. unidentified

Nephtys cornuta franciscana

Ninoe gemma

ol igochaete

Polydora cornuta

Tharyx mutifilis

TOTAL ANNELIDS

ARTHROPODS :

Ampel isca abdita

Corophium al ienense

cumacea sp. 1

Eusarsiella sp. 1

TOTAL ARTHROPODS

MOLLUSCS:

bivalve sp. 1

bivalve sp. 2

bivalve sp. unidentified

TOTAL MOLLUSCS

TOTAL INFAUNA
Number of individuals $/ 10 \mathrm{~cm}$ diameter core sample

\begin{tabular}{llllllllll}
\hline \multicolumn{1}{c}{ Repl icate Number } \\
\hline$\underline{5}$ & $\underline{6}$ & $\underline{7}$ & $\underline{9}$ & $\underline{13}$ & $\underline{14}$ & $\underline{15}$ & Mean & $\underline{\text { SDEV }}$ & CV
\end{tabular}

$\begin{array}{rrrrrrrrrr}1 & 0 & 0 & 0 & 0 & 0 & 0 & 0.1 & 0.3 & 2.45 \\ 0 & 0 & 0 & 0 & 1 & 0 & 0 & 0.1 & 0.3 & 2.45 \\ 0 & 0 & 0 & 0 & 1 & 0 & 0 & 0.1 & 0.3 & 2.45 \\ 0 & 1 & 0 & 1 & 2 & 1 & 0 & 0.7 & 0.7 & 0.98 \\ 0 & 0 & 1 & 0 & 0 & 0 & 0 & 0.1 & 0.3 & 2.45 \\ 1 & 2 & 1 & 2 & 0 & 0 & 1 & 1.0 & 0.8 & 0.76 \\ 0 & 1 & 0 & 0 & 0 & 1 & 1 & 0.4 & 0.5 & 1.15 \\ 0 & 1 & 0 & 0 & 0 & 0 & 0 & 0.1 & 0.3 & 2.45 \\ 0 & 0 & 0 & 0 & 0 & 0 & 1 & 0.1 & 0.3 & 2.45 \\ 4 & 0 & 4 & 1 & 0 & 5 & 0 & 2.0 & 2.1 & 1.04 \\ 0 & 1 & 0 & 0 & 0 & 0 & 0 & 0.1 & 0.3 & 2.45 \\ 0 & 1 & 0 & 0 & 0 & 0 & 0 & 0.1 & 0.3 & 2.45 \\ 8 & 4 & 4 & 7 & 2 & 10 & 1 & 5.1 & 3.0 & 0.59 \\ 0 & 2 & 0 & 1 & 0 & 0 & 0 & 0.4 & 0.7 & 1.70 \\ 0 & 1 & 0 & 0 & 0 & 0 & 1 & 0.3 & 0.5 & 1.58 \\ & & & & & & & & & \\ 14 & 14 & 10 & 12 & 6 & 17 & 5 & 11.1 & 4.1 & 0.37\end{array}$

$\begin{array}{rrrrrrrrrr}0 & 3 & 1 & 0 & 0 & 0 & 0 & 0.6 & 1.0 & 1.84 \\ 4 & 8 & 4 & 4 & 10 & 5 & 3 & 5.4 & 2.4 & 0.44 \\ 0 & 0 & 0 & 0 & 1 & 0 & 0 & 0.1 & 0.3 & 2.45 \\ 1 & 1 & 0 & 0 & 1 & 2 & 1 & 0.9 & 0.6 & 0.75 \\ 5 & 12 & 5 & 4 & 12 & 7 & 4 & 7.0 & 3.3 & 0.47\end{array}$

$\begin{array}{rrrrrrrrrr}13 & 8 & 7 & 13 & 9 & 5 & 7 & 8.9 & 2.8 & 0.32 \\ 0 & 2 & 0 & 4 & 0 & 7 & 0 & 1.9 & 2.5 & 1.36 \\ 0 & 5 & 0 & 0 & 0 & 0 & 0 & 0.7 & 1.7 & 2.45 \\ 13 & 15 & 7 & 17 & 9 & 12 & 7 & 11.4 & 3.6 & 0.32 \\ 32 & 41 & 22 & 33 & 27 & 36 & 16 & 29.6 & 7.9 & 0.27\end{array}$


IABLE 3.4. Infauna Counts from Station G10

$\underline{T a x a}$

ANNELIDS:

Cirriformia spirabrancha

Exogone lourei

Exogone spp. unidentified

Glycinde polygnatha

Glycinde spp. unidentified

Meiodorvillea sp.

ol igochaete

Polycirrus californicus

Scolelepis squamata

Syllides longocirrata

TOTAL ANNELIDS

Number of individuals $/ 10 \mathrm{~cm}$ diameter core sample

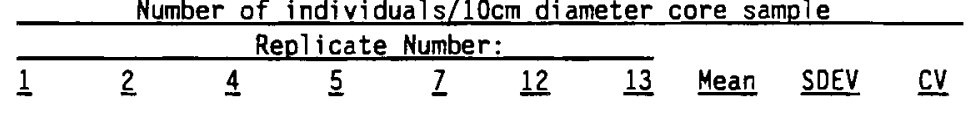

$\begin{array}{rrrrrrrrrr}1 & 0 & 0 & 0 & 0 & 0 & 0 & 0.1 & 0.3 & 2.45 \\ 4 & 20 & 27 & 5 & 0 & 15 & 23 & 13.4 & 9.7 & 0.72 \\ 0 & 0 & 1 & 0 & 0 & 0 & 0 & 0.1 & 0.3 & 2.45 \\ 0 & 0 & 0 & 0 & 0 & 1 & 0 & 0.1 & 0.3 & 2.45 \\ 0 & 1 & 0 & 0 & 0 & 0 & 0 & 0.1 & 0.3 & 2.45 \\ 0 & 0 & 0 & 0 & 0 & 1 & 0 & 0.1 & 0.3 & 2.45 \\ 0 & 4 & 1 & 0 & 0 & 2 & 0 & 1.0 & 1.4 & 1.41 \\ 0 & 1 & 1 & 1 & 0 & 0 & 1 & 0.6 & 0.5 & 0.87 \\ 0 & 0 & 1 & 0 & 0 & 0 & 0 & 0.1 & 0.3 & 2.45 \\ 0 & 0 & 1 & 0 & 0 & 0 & 0 & 0.1 & 0.3 & 2.45 \\ 5 & 26 & 32 & 6 & 0 & 19 & 24 & 16.0 & 11.4 & 0.71\end{array}$

ARTHROPODS:

Ampelisca abdita

Ampelisca sp.

Photis sp. 1

TOTAL ARTHROPODS

\begin{tabular}{|c|c|c|c|c|c|c|c|c|c|}
\hline 1 & 0 & 0 & 3 & 2 & 0 & 0 & 0.9 & 1.1 & 1.31 \\
\hline 0 & 0 & 0 & 0 & 2 & 0 & 0 & 0.3 & 0.7 & 2.45 \\
\hline 0 & 0 & 0 & 1 & 0 & 0 & 0 & 0.1 & 0.3 & 2.45 \\
\hline & 0 & 0 & 4 & 4 & 0 & 0 & 1.3 & 1.7 & 1.36 \\
\hline
\end{tabular}

MOLLUSCS:

bivalve sp. 6

bivalve sp, unidentified (broke

Germa gemma

$\begin{array}{rrrrrrrrrr}0 & 0 & 1 & 0 & 1 & 0 & 0 & 0.3 & 0.5 & 1.58 \\ 0 & 0 & 1 & 0 & 0 & 0 & 0 & 0.1 & 0.3 & 2.45 \\ 0 & 0 & 38 & 0 & 21 & 0 & 0 & 8.4 & 14.1 & 1.67 \\ 0 & 0 & 40 & 0 & 22 & 0 & 0 & 8.9 & 14.8 & 1.67 \\ & & & & & & & & & \\ 6 & 26 & 72 & 10 & 26 & 19 & 24 & 26.1 & 20.1 & 0.77\end{array}$

TOTAL INFAUNA

TOTAL MOLLUSCS

120




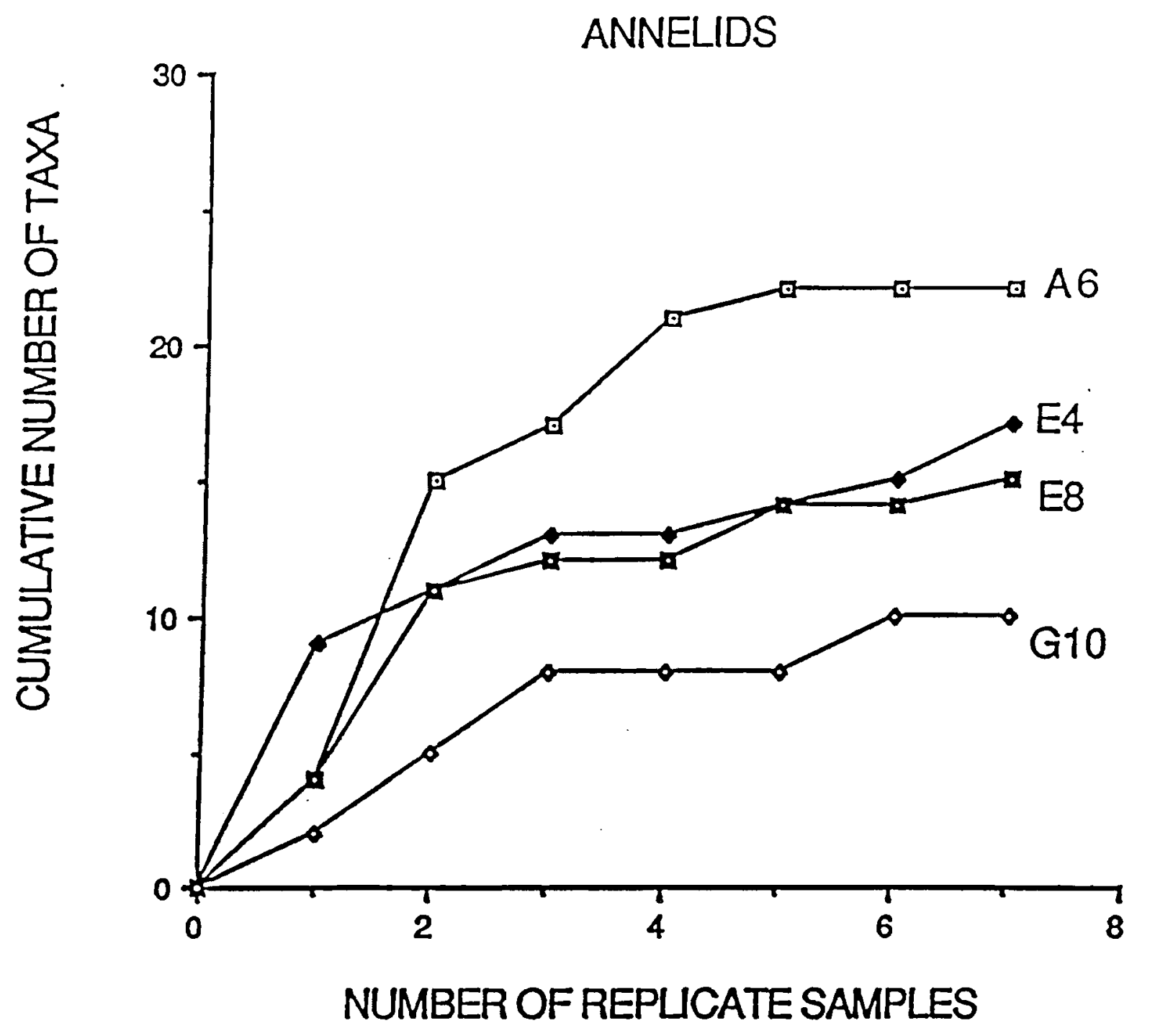

FIGURE 3.1. Cumulative Number of Infaunal Annelid Taxa Versus Number of Replicate Samples From Each of the Four Stations 


\subsubsection{Total Organic Carbon, 0il and Grease, and Total Petroleum Hydrocarbons}

The results for conventional analyses, including percent solids, TOC, $0 i 1$ and grease and total petroleum hydrocarbons, are presented in Table 3.5. All detection limits were met for conventional parameters for sediments. Method blanks for TOC, 011 and grease and TPH showed little or no contamination. Matrix spike data for TOC was acceptable, ranging from 50 to $88 \%$. Analytical replicated analyses for percent solids and TOC showed good agreement with RPDs, ranging from 0.06 to $8 \%$. RPDs for $0 i 1$ and grease and TPH were higher ranging from 30 to $40 \%$. These are outside of the DQOs for these parameters, but the level of hydrocarbons measured is relatively low for these samples, which influences the RPD values. This difference is not significant.

\subsubsection{Grain Size}

Grain size results are presented in Table 3.6 and Figures 3.2 and 3.3 . One analytical replicate sample was analyzed. There was good agreement between replicate analyses as seen by RPD values ranging from 0 to $29 \%$. The slightly higher RPD values observed for some of the fractions are a result of the low percentage of material involved in those fractions, which exaggerates the percent difference between replicates. Method blanks, surrogates, spikes, and SRMs do not apply to grain size analyses.

Figures 3.2 and 3.3 represent plots of grain size fractions for a 11 samples. Note that the classifications given at the bottom of each table are based on ASTM Method 2487 size fractions. These present the gravel as greater than $4.75 \mathrm{~mm}$ and the sand fraction as less than $4.75 \mathrm{~mm}$ and greater than 62.5 $\mathrm{mm}$. These are somewhat different from those reported in Table 3.6.

\subsubsection{Metals}

Results for metals analyses of sediment samples are presented in Table 3.7. Detection limit goals for all metals were met. The procedural blank for sediment analyses showed no contamination of metals with the exception of low levels of mercury, $0.007 \mathrm{mg} / \mathrm{kg}$, well below any sample concentration. Blank data are not applicable for the metals run by $\mathrm{X}$-ray fluorescence (As, $\mathrm{Cr}, \mathrm{Cu}$, $\mathrm{Ni}, \mathrm{Pb}$, and $\mathrm{Zn})$. Analytical replicate analyses performed on sample STA-E8-R2 showed generally good agreement. RPD values ranged from 0 to $37 \%$ with RPDs 


\section{TABLE 3.5. Sediment Conventionals}

\begin{tabular}{|c|c|c|c|c|}
\hline \multirow[b]{2}{*}{$\begin{array}{l}\text { Sponsor } \\
\text { Code }\end{array}$} & \multicolumn{4}{|c|}{ (concentrations in $\mathrm{mg} / \mathrm{kg}$ dry $w \mathrm{t}$ ) } \\
\hline & $\%$ Solids & $\begin{array}{c}\text { TOC } \\
\mathbf{x} \text { dry wt. } \\
\end{array}$ & $0 i 1$ and Grease & $\begin{array}{c}\text { Total Petroleum } \\
\text { Hydrocarbons }\end{array}$ \\
\hline TARGET DETECTION LIMITS: & N/A & 0.1 & 20 & 20 \\
\hline STA-E8-R2 REP 1 & $35.6 \%$ & 1.29 & 59 & 52 \\
\hline STA-E8-R2 REP 2 & N/A & 1.19 & 80 & 34 \\
\hline STA-E4-R2 & $47.5 \%$ & 0.96 & 70 & 48 \\
\hline STA-A6-R2 & $64.5 \%$ & 0.41 & 31 & 20 \\
\hline STA-G10-R2 REP 1 & $80.9 \%$ & 0.04 & 6.1 & 2.1 \\
\hline STA-G10-R2 REP 2 & $81.0 \%$ & N/A & N/A & N/A \\
\hline
\end{tabular}

$U$ indicates parameter not detected at detection limit shown.

N/A indicates not applicable.

Quality Control Data for Sediment Conventionals

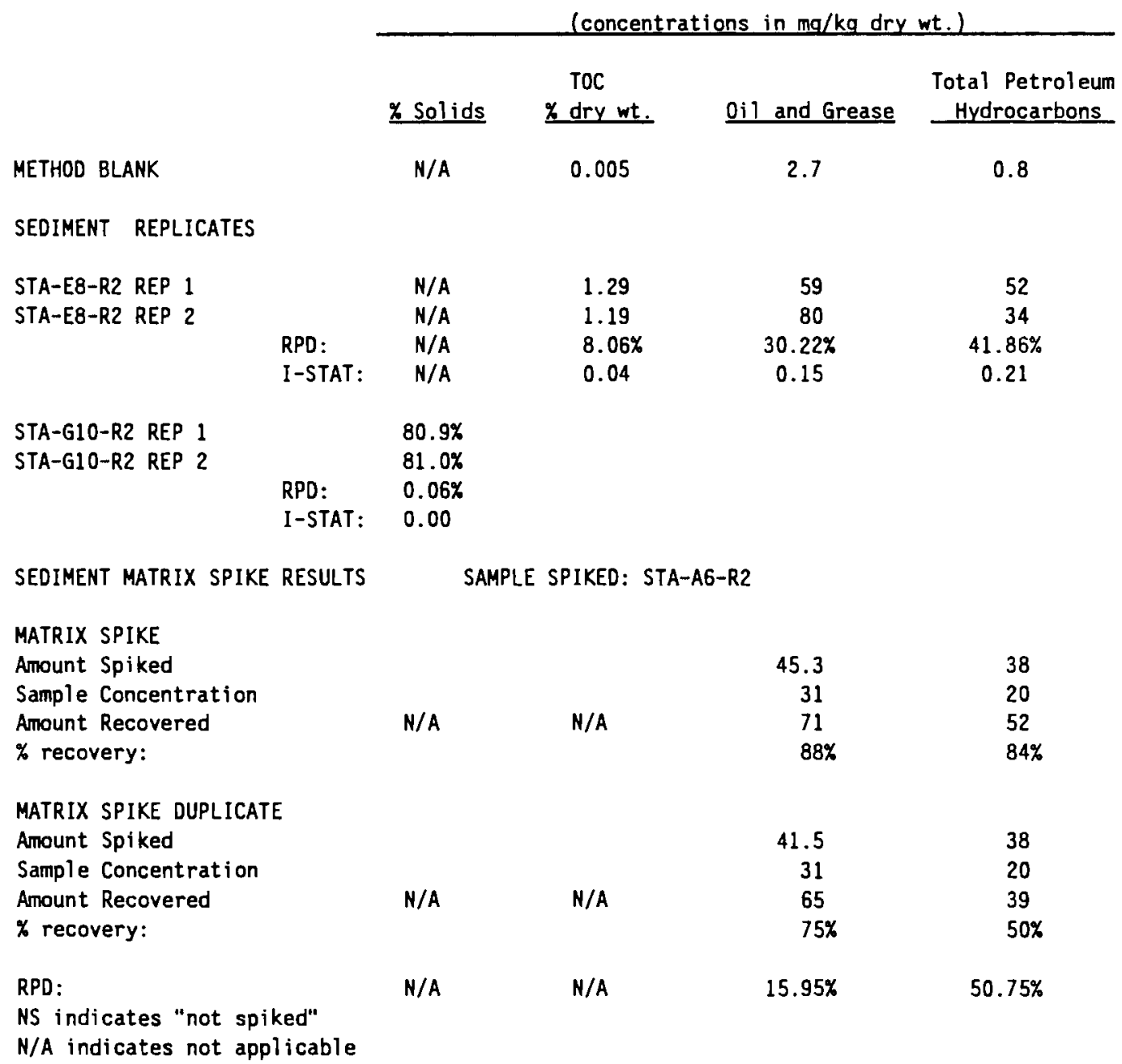


TABLE 3.6. Grain Size

\begin{tabular}{|c|c|c|c|c|c|c|c|c|c|c|c|c|c|c|c|}
\hline \multirow{2}{*}{$\begin{array}{l}\text { Gravel } \\
-2.25\end{array}$} & \multicolumn{6}{|c|}{ Sand } & \multicolumn{6}{|c|}{ Silt } & \multicolumn{3}{|c|}{ Clay } \\
\hline & -1 & 0.25 & 1.25 & 2 & & 3.75 & 4 & 4.5 & 5 & 5.5 & 6 & 7 & 8 & 9 & 10 \\
\hline 4 & 10 & 20 & 40 & 60 & 100 & 200 & 230 & & & & & & & & \\
\hline 4.75 & 2 & 0.85 & 0.425 & 0.25 & 0.106 & 0.075 & 0.062 & 0.048 & 0.031 & 0.022 & 0.016 & 0.00 & 0.004 & 0.002 & 0.001 \\
\hline
\end{tabular}

phi :

sieve No.:

grain size (in $\mathrm{mm}$.$) :$

$\begin{array}{rrrrrrr}100 & 100 & 100 & 100 & 100 & 100 & 99 \\ 100 & 100 & 100 & 100 & 100 & 99 & 98 \\ 91 & 80 & 71 & 67 & 64 & 58 & 48 \\ 100 & 99 & 98 & 96 & 59 & 3 & 1 \\ 100 & 100 & 98 & 97 & 61 & 4 & 1\end{array}$

$\begin{array}{rrr}99 & 99 & 98 \\ 83 & 79 & 78 \\ 43 & 41 & 40 \\ 0 & 0 & 0 \\ 0 & 0 & 0\end{array}$

ANALYTICAL REPLICATES:

$\omega$
io
STA-G10-R3 REP1

$\begin{array}{rrrrrrr}100 & 99 & 98 & 96 & 59 & 3 & 1 \\ 100 & 100 & 98 & 97 & 61 & 4 & 1 \\ 0 \% & 1 \% & 0 \% & 1 \% & 3 \% & 29 \% & 0 \% \\ 0.00 & 0.01 & 0.00 & 0.01 & 0.02 & 0.14 & 0.00\end{array}$

$\begin{array}{lllllllll}0 & 0 & 0 & 0 & 0 & 0 & 0 & 0 & 0 \\ 0 & 0 & 0 & 0 & 0 & 0 & 0 & 0 & 0 \\ 0 \% & 0 \% & 0 \% & 0 \% & 0 \% & 0 \% & 0 \% & 0 \% & 0 \% \\ 0 & 0 & 0 & 0 & 0 & 0 & 0 & 0 & 0\end{array}$

SUMMARY OF GRAIN SIZE DISTRIBUTION IN BAY FARM SEDIMENTS

$\begin{array}{llll}\text { PERCENT } & \text { PERCENT } & \text { PERCENT } & \text { PERCENT } \\ \text { GRAVEL } & \text { SAND } & \text { SILT } & \text { CLAY } \\ & 2000 \text { to } & 62.5 \text { to } & \\ >2000 \text { um } & 62.5 \mathrm{um} & 3.9 \mathrm{um} & <3.9 \mathrm{um}\end{array}$

$\begin{array}{rrrr}0 & 1 & 28 & 71 \\ 0 & 17 & 28 & 55 \\ 20 & 37 & 12 & 31 \\ 2 & 98 & 0 & 0 \\ 2 & 98 & 0 & 0\end{array}$


TABLE 3.7. Sediment Metals

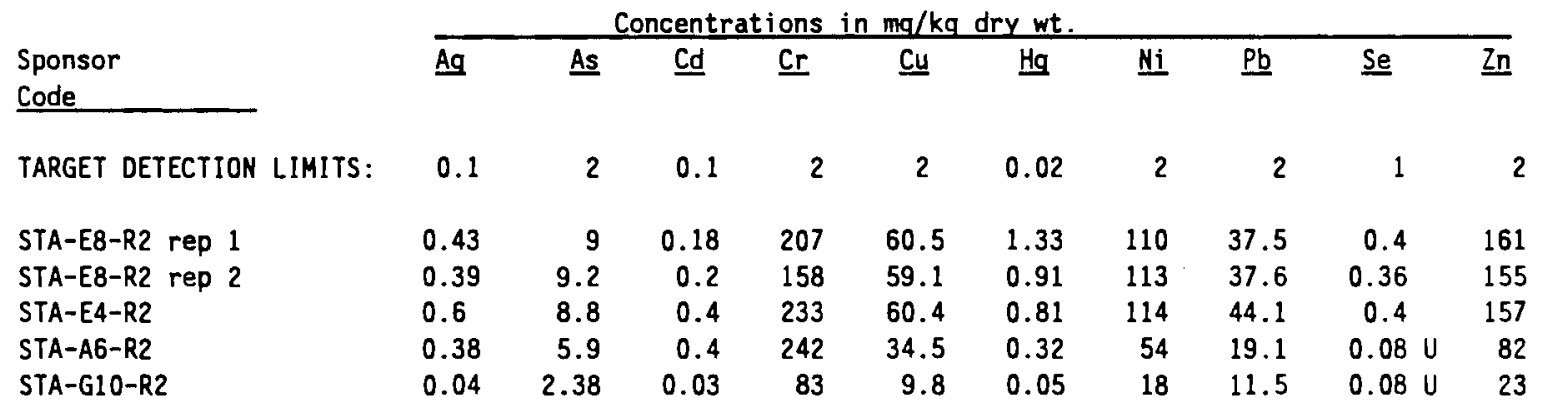

$U$ indicates parameter not detected at detection 1 imit shown

Quality control results for sediment metals

$\begin{array}{ccccccccccc} & \underline{\mathrm{Aq}} & \underline{\mathrm{As}} & \underline{\mathrm{Cd}} & \underline{\mathrm{Cr}} & \underline{\mathrm{Cu}} & \underline{\mathrm{Hg}} & \underline{\mathrm{Ni}} & \underline{\mathrm{Pb}} & \underline{\mathrm{Se}} & \underline{\mathrm{Zn}} \\ \text { PROCEDURAL BLANK: } & 0.005 & \mathrm{~N} / \mathrm{A} & 0.00 \mathrm{U} & \mathrm{N} / \mathrm{A} & \mathrm{N} / \mathrm{A} & 0.00 & \mathrm{~N} / \mathrm{A} & \mathrm{N} / \mathrm{A} & 0.08 \mathrm{U} & \mathrm{N} / \mathrm{A}\end{array}$

\section{ANALYTICAL REPLICATE}

$\begin{array}{lcccccccccc}\text { STA-E8-R2 rep 1 } & 0.43 & 9 & 0.18 & 207.0 & 60.5 & 1.33 & 109.9 & 37.5 & 0.4 & 160.6 \\ \text { STA-E8-R2 rep 2 } & 0.39 & 9.2 & 0.2 & 158.0 & 59.1 & 0.91 & 113 & 37.6 & 0.36 & 154.6 \\ & & 10 \% & 2 \% & 11 \% & 27 \% & 2 \% & 37 \% & 3 \% & 0 \% & 11 \% \\ \text { RPD: } & 0.05 & 0.01 & 0.05 & 0.13 & 0.01 & 0.19 & 0.01 & 0.00 & 0.05 & 0.02\end{array}$

STANDARD REFERENCE MATERIAL:

\begin{tabular}{|c|c|c|c|c|c|c|c|c|c|c|}
\hline $\begin{array}{l}\text { BCSS-1 } \\
\quad \text { Certified Value: }\end{array}$ & na & na & $\begin{array}{r}0.27 \\
0.25 \\
\pm 0.8\end{array}$ & na & na & $\begin{array}{l}0.13 \\
\text { NC }\end{array}$ & na & na & $\begin{array}{l}0.47 \\
0.43 \\
0.06\end{array}$ & na \\
\hline $\begin{array}{l}\text { MESS-1 } \\
\quad \text { Certified Value: }\end{array}$ & $\begin{array}{r}0.106 \\
N C \\
-\end{array}$ & $\begin{array}{r}10.3 \\
10.6 \\
+1.2\end{array}$ & $\begin{array}{r}0.71 \\
0.59 \\
\pm 0.1\end{array}$ & na & na & $\begin{array}{r}0.17 \\
0.17 \\
\text { na }\end{array}$ & na & na & $\begin{array}{r}0.4 \\
0.34 \\
0.06\end{array}$ & na \\
\hline $\begin{array}{l}\text { SRM } 1646 \\
\text { Certified Value: }\end{array}$ & $\begin{array}{r}0.095 \\
\text { NC }\end{array}$ & $\begin{array}{r}10.8 \\
11.6 \\
+1.3\end{array}$ & $\begin{array}{r}0.37 \\
0.36 \\
+0.07\end{array}$ & $\begin{array}{r}92 \star \\
76.0 \\
\pm 3\end{array}$ & $\begin{array}{r}19.1 \\
18.0 \\
\pm 3\end{array}$ & $\begin{array}{l}\text { na } \\
\text { NC }\end{array}$ & $\begin{array}{r}30.5 \\
32.0 \\
\pm 3\end{array}$ & $\begin{array}{r}28.0 \\
28.2 \\
+1.8\end{array}$ & $\begin{array}{r}0.61 \\
N / C\end{array}$ & $\begin{array}{r}131.8 \\
138 \\
\pm 6\end{array}$ \\
\hline
\end{tabular}

* values outside of range of certified value.

N/A indicates not applicable

na indicates not analyzed

NC indicates analyte not certified in SRM 


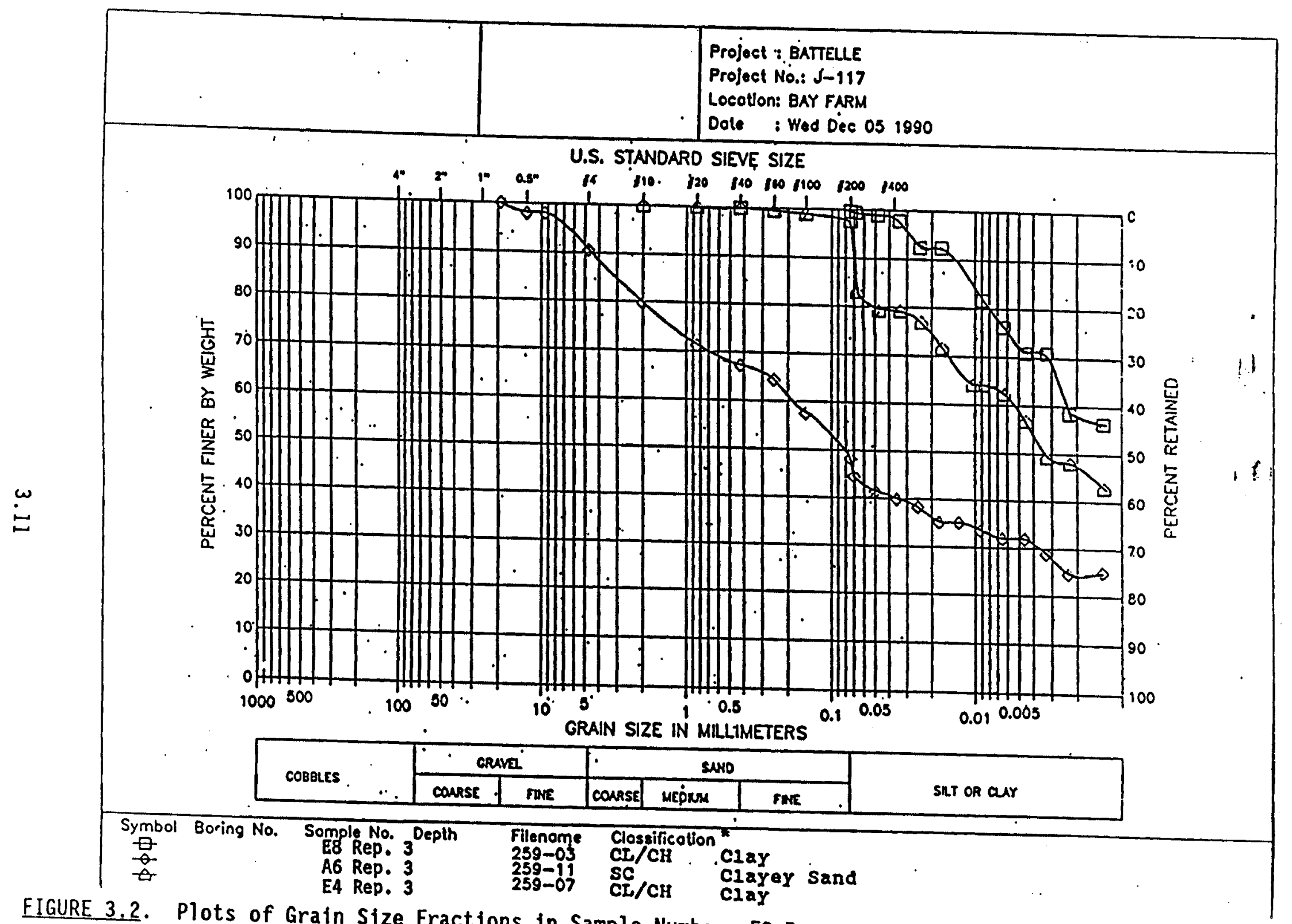

EIGURE 3.2. Plots of Grain Size Fractions in Sample Numbers E8 Rep. 3, A6, Rep. 3, and E4 Rep. 3.

(Note: size fractions are those listed in ASTM Method 2487, ie., gravel greater than $4.75 \mathrm{~mm}$.) 


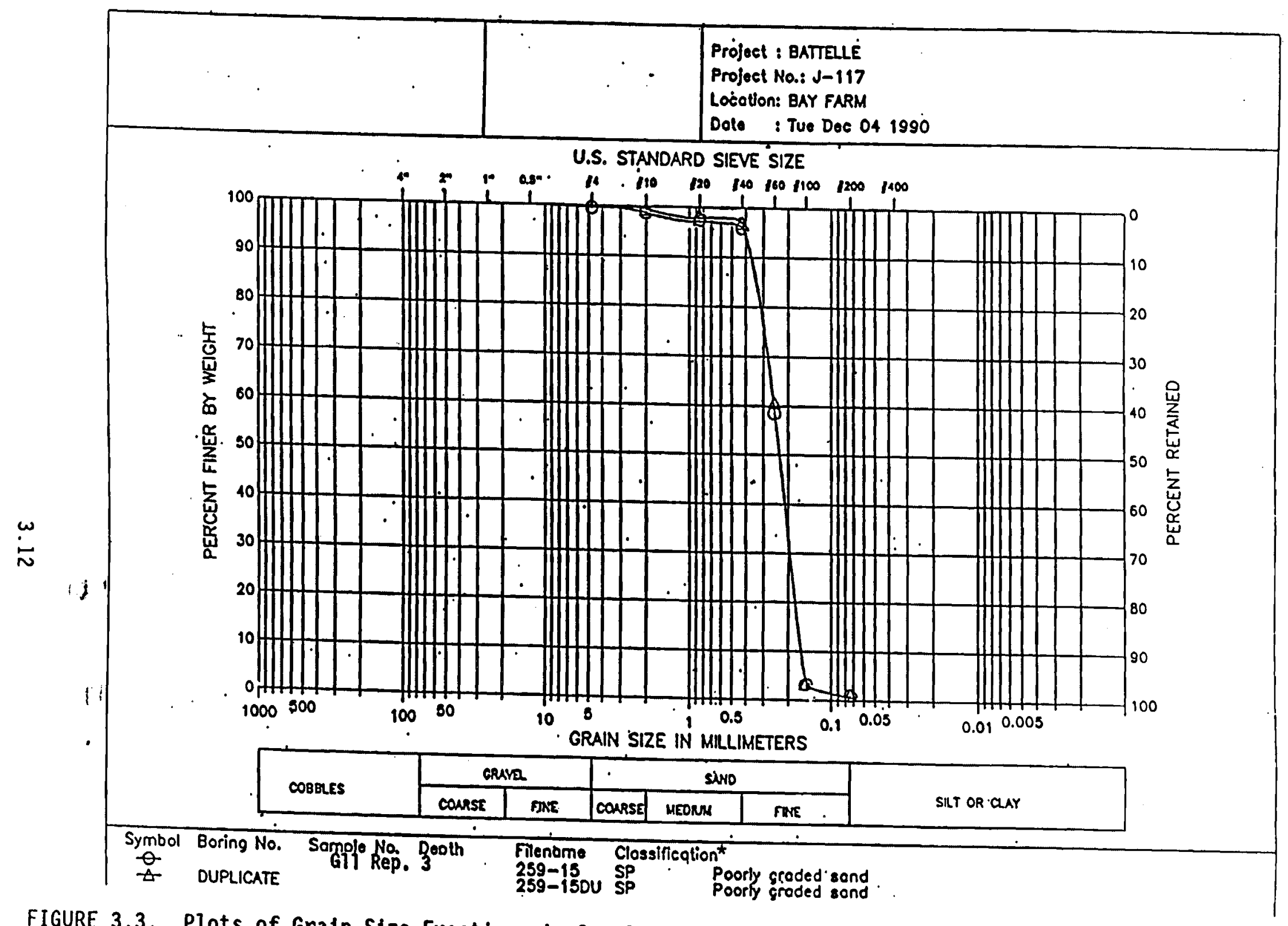
EIGURE 3.3. Plots of Grain Size Fractions in Sample Number Gil Rep. 3 . (Note: size fractions are those
listed in ASTM Method 2487, 1.e., gravel greater than $4.75 \mathrm{~mm}$. .) 
for 8 of the 10 metals at less than 15\%. RPDs for two of the metals, $\mathrm{Cr}$ and $\mathrm{Hg}$, minimally exceeded control limits for precision.

Three SRMs (BCSS-1, MESS-1, and SRM 1646) were analyzed to encompass all metals analyzed. Comparison of measured and certified values resulted in one metal value, chromium, outside of the certified concentration range.

\subsubsection{Butyltins}

Butyltin results are presented in Table 3.8. Detection limit goals were met for all butyltins. Tributyltin was detected in the method blank at 1.5 $\mu \mathrm{g} / \mathrm{kg}$. Surrogate recoveries for all samples analyzed ranged from 45 to $86 \%$. Matrix spike recoveries ranged from $49 \%$ for tetrabutyltin to $84 \%$ for dibutyltin. These are within acceptable ranges. RPDs for one analytical replicate analyzed ranged from 0 to $13 \%$ for compounds detected. The RPD for monobutyltin was high, but the levels compared were near detection limits.

One SRM (PACS-1) was analyzed for tri- and dibutyltin. Taking into the surrogate recovery of $87 \%$, the RPDs between the measured values and the certified values were approximately $30 \%$. This level slightly exceeds the control limit of 20; however, the certification of the material may have been slightly biased by correcting recoveries for the internal standard, tripropyltin, which may not accurately reflect tributyltin response. At the time of writing this report, a new surrogate compound, tripentyltin, is being evaluated.

\subsubsection{Total Phenols, Phenol, and Substituted Phenols}

Phenol results are presented in Table 3.9. To meet detection limit goals, total phenols, chlorinated and other phenols in sediments were measured by three different methods as described above. All detection limits were met for phenols with the exception of total phenols. Detection limits for total phenol ranged from 0.5 to $1.4 \mathrm{mg} / \mathrm{kg}$, and the goal stated 0.02 to $0.1 \mathrm{mg} / \mathrm{kg}$. The initial detection limit goals were too low for the method employed. No phenols were detected in any of the method blanks.

Surrogate recoveries for total phenols analyzed by GC were somewhat high ranging from 134 to $159 \%$. Phenols measured by GC/FID ranged from 77 to $85 \%$. To date, no control limits have been established for these methods, although 
TABLE 3.8. Sediment Butyltins (concentrations in $\mu \mathrm{g} / \mathrm{kg}$ dry wt.)

\begin{tabular}{|c|c|c|c|c|c|}
\hline $\begin{array}{l}\text { Sponsor } \\
\text { Code }\end{array}$ & $\begin{array}{l}\text { Tetrabutyl- } \\
\text { tin } \\
\end{array}$ & $\begin{array}{l}\text { Tributyl- } \\
\text { tin } \\
\end{array}$ & $\begin{array}{l}\text { Dibutyl- } \\
\text { tin } \\
\end{array}$ & $\begin{array}{l}\text { Monobutyl- } \\
\text { tin } \\
\end{array}$ & $\begin{array}{l}\text { Surrogat } \\
\text { Recovery } \\
\text { Propylti }\end{array}$ \\
\hline TARGET DETECTION LIMIT: & 10 & 10 & 10 & 10 & $40-140 \%$ \\
\hline STA-E8-R2 REP 1 & $1.5 \mathrm{U}$ & $4.9 B$ & 2.9 & 2.1 & $45 \%$ \\
\hline STA-E8-R2 REP 2 & $1.5 \mathrm{U}$ & $4.3 \mathrm{~B}$ & 3.2 & 1.3 & $74 \%$ \\
\hline STA-E4-R2 & $1.1 \mathrm{U}$ & $3 \mathrm{~B}$ & 3.3 & 3.7 & $70 \%$ \\
\hline STA-A6-R2 & $1 U$ & $4.4 B$ & 1.8 & 0.9 & $69 \%$ \\
\hline STA-G1O-R2 & $0.7 U$ & $0.9 \mathrm{~B}$ & 2.9 & 0.6 & $86 \%$ \\
\hline
\end{tabular}

$U$ indicates parameter not detected at detection limit shown.

$B$ indicates anlayte was detected in the method blank.

Quality Control Results for Butyltins in Sediment

\begin{tabular}{|c|c|c|c|c|c|}
\hline & $\begin{array}{l}\text { Tetrabutyl - } \\
\text { tin } \\
\end{array}$ & $\begin{array}{l}\text { Tributyl- } \\
\text { tin } \\
\end{array}$ & $\begin{array}{l}\text { Dibutyl- } \\
\text { tin } \\
\end{array}$ & $\begin{array}{l}\text { Monobutyl - } \\
\text { tin } \\
\end{array}$ & $\begin{array}{l}\text { Surrogat } \\
\text { Recovery } \\
\text { Propyltin }\end{array}$ \\
\hline METHOD BLANK & $1.1 \mathrm{U}$ & 1.5 & $1 \mathrm{U}$ & 1 & $60 \%$ \\
\hline MATRIX SPIKE RECOVERY & SAMPLE SPIKED: & STA-G10-R2 & & & \\
\hline Sample Concentration & $0.7 \mathrm{U}$ & $0.9 \mathrm{~B}$ & 2.9 & 0.6 & $86 \%$ \\
\hline Amount Spiked & 123 & 123 & 123 & & \\
\hline Amount Recovered & 59.9 & 93.9 & 102 & NS & $47 \%$ \\
\hline \% recovery: & $49 \%$ & $76 \%$ & $84 \%$ & - & \\
\hline
\end{tabular}

REPLICATE ANALYSIS

$\begin{array}{lccccc}\text { STA-EB-R2 } & 1.5 U & 4.9 & 2.9 & 2.1 & 45 \% \\ \text { STA-EB-R2 } & 1.5 U & 4.3 & 3.2 & 1.3 & 74 \% \\ \text { RPD: } & 0 \% & 13 \% & 10 \% & 47 \% & \\ \text { I-STAT : } & 0.00 & 0.07 & 0.05 & 0.24\end{array}$

STANDARD REFERENCE MATERIAL

$\begin{array}{lcccc}\text { PACS-1 } & \text { NC } & 764.2 & 730.6 & \text { NS } \\ \text { certified value: } & \text { NC } & 1270 & 1160 & 87 \% \\ & & (+/-220) & (+/-180) & \\ \text { NS indicates "not spiked" } & & \\ U \text { indicates parameter not detected at detection limit shown } \\ \text { NC indicates not certified in SRM }\end{array}$


TABLE 3.9. Sediment Phenols (concentrations in mg/kg dry wt.)

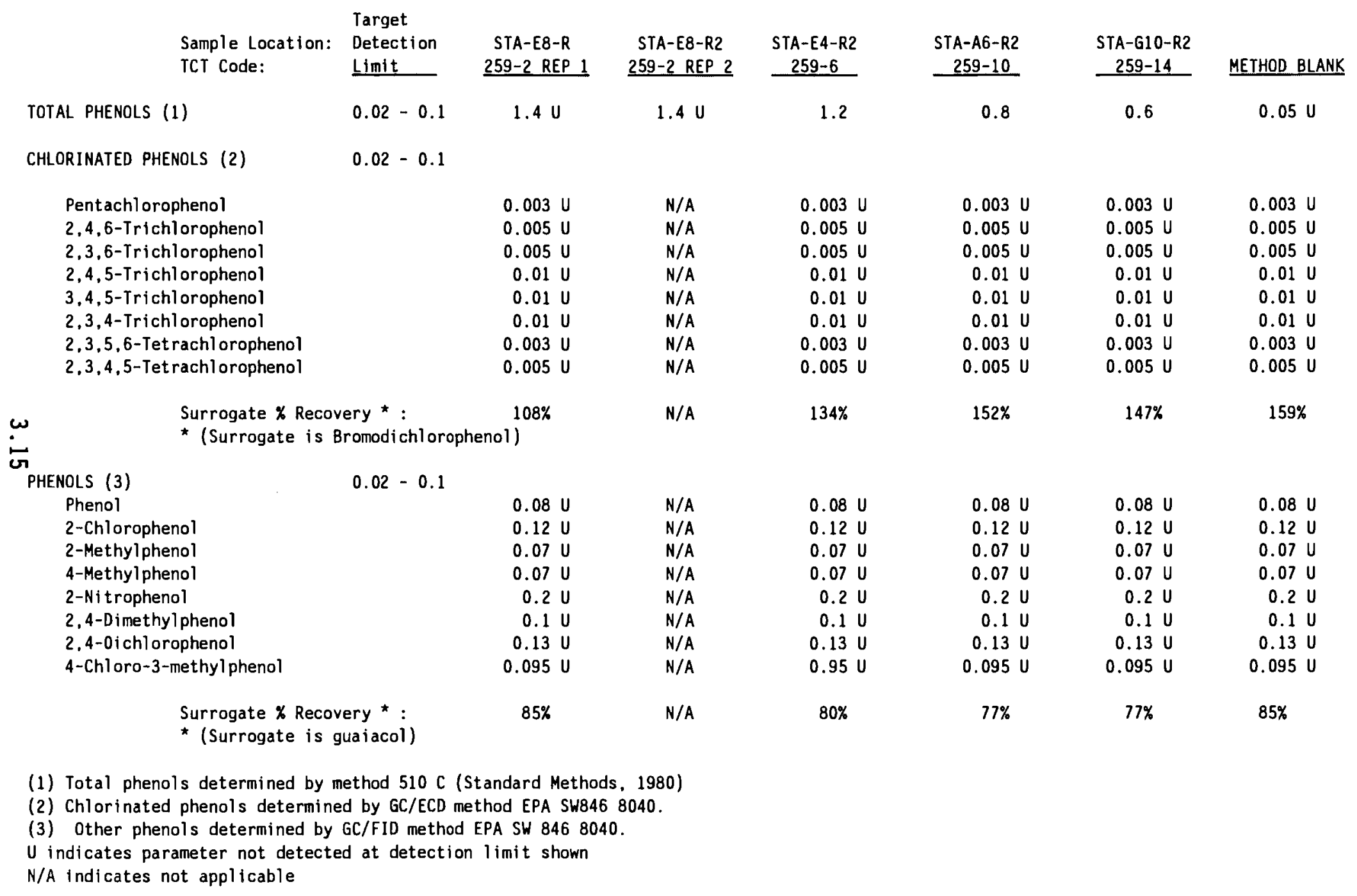




\section{TABLE 3.9. (contd)}

Quality Control Results for Sediment Phenols

SEDIMENT ANALYTICAL REPLICATES

\begin{tabular}{|c|c|}
\hline $\begin{array}{l}\text { Sponsor } \\
\text { Code } \\
\end{array}$ & $\begin{array}{l}\text { Total Phenol } \\
\mathrm{mg} / \mathrm{kg} \text { dry wt }\end{array}$ \\
\hline STA-E8-R2 & $1.4 \mathrm{U}$ \\
\hline STA-E8-R2 & $1.4 \mathrm{U}$ \\
\hline & $\begin{array}{r}\text { ERR } \\
0\end{array}$ \\
\hline
\end{tabular}

SEDIMENT MATRIX SPIKE RESULTS

(spiked sample : STA-E8)

家

MATRIX SPIKE RECOVERY

Sample Concentration

Amount Splked

Amount Recovered

\% recovery:

MATRIX SPIKE DUPLICATE

Amount Spiked

$\%$ recovery:

RPD :

N/A

N/A

Total Phenols

\begin{tabular}{cc} 
& GC/FID P \\
\hline Phenol & $\begin{array}{c}2.4-D \text { - } 1 \text { methyl } \\
\text { phenol }\end{array}$ \\
\cline { 3 - 3 } $0.08 \mathrm{U}$ & $0.1 \mathrm{U}$ \\
0.725 & 0.725 \\
0.65 & 0.61 \\
$90 \%$ & $84 \%$ \\
& \\
0.57 & 0.52 \\
$79 \%$ & $72 \%$ \\
$13 \%$ & $15 \%$
\end{tabular}

GC/ECD Phenols

Pentachlorophenol

$0.003 \mathrm{U}$

0.112

0.107

$97 \%$

$74 \% \quad 95 \%$

$15 \% \quad 2 \%$ 
the laboratory performing these analyses (ARI) is in the process of collecting data to establish these limits. No surrogates are measured for the total phenol analysis.

Matrix spikes showed good recoveries for all methods with recoveries ranging from a low of $57 \%$ for total phenols and a range of 72 to $97 \%$ for the GC methods. RPD between matrix spikes and matrix spike duplicates run by the GC methods were 10w, ranging from 2 to 15\%. Analytical replicates were only run for total phenols. No phenols were detected in either sample. No sediment SRMs are available for phenol analyses.

\subsubsection{Polynuclear Aromatic Hydrocarbons}

Results of PAH analyses for sediments are presented in Table 3.10.

Detection limit goals were met for all PAH compounds analyzed. Analyses for three of the four samples were performed on diluted samples $(1: 10)$. Surrogate recoveries for all samples ranged from 50 to $117 \%$. These were within acceptable limits of recovery. Matrix spike recoveries were acceptable for all compounds spiked.

Initially, the results for the MS and MSD were found to be unacceptable because of high percent recoveries ranging from approximately 100 to $375 \%$. This was thought to be as a result of high native concentrations in the sample, which were not reflected by the initial analysis of that sample (STAE4-R2). This sample was subsequently reanalyzed and reported (STA-E4-R2-RE). Based on the previous matrix spiked data, and the current results for this sample, it is likely that the sample is non-homogeneous, which has resulted in variations in PAH concentrations.

In addition, another matrix spike/matrix spike duplicate analysis was performed on a sample of lower native concentrations (STA-G10-R2); the results were found acceptable and are presented in this report.

\subsubsection{Chlorinated Pesticides and Polychlorinated Biphenyls}

The PCB and chlorinated pesticide data for sediments are presented in Tables 3.11 and 3.12 , respectively. All detection limit goals for PCBs and pesticides were met. No compounds were detected above detection limit goals in the method blank. Surrogate recoveries for all samples were greater than 90\%. Matrix spike and matrix spike duplicate results for pesticides ranged 
TABLE 3.10. Sediment Polynuclear Aromatic Hydrocarbons

\begin{tabular}{lllllll} 
(Concentrations in ug/kg dry wt) \\
\cline { 2 - 3 } & Naph- & Acenaph- & Acenaph- & Phenan- & Anthra- & Flouran- \\
Name & thalene & thylene & thene & Flourene & threne & Pyrene
\end{tabular}

TARGET DETECTION LIMITS:

$20 \mathrm{ug} / \mathrm{kg}$

\begin{tabular}{|c|c|c|c|c|c|c|c|c|}
\hline STA-EB-R2 REP 1* & $44 \mathrm{U}$ & $6.6 U$ & $17 U$ & $13 \mathrm{U}$ & 63 & 10 & 220 & 290 \\
\hline STA-E8-R2 REP 2* & $44 \mathrm{U}$ & $6.7 \mathrm{U}$ & $17 \mathrm{U}$ & $13 \mathrm{U}$ & 58 & 10 & 210 & 280 \\
\hline STA-E4-R2 ${ }^{\star}$ & $41 \mathrm{U}$ & $6.2 \mathrm{U}$ & $15 \mathrm{U}$ & $12 \mathrm{U}$ & 120 & 18 & 280 & 360 \\
\hline STA-E4-R2 RE $(1)^{*}$ & $22 \mathrm{U}$ & 37.7 & 21.6 & 17.7 & 230 & 68.4 & 549 & 690 \\
\hline STA-A6-R2* & $30 \mathrm{U}$ & $4.6 \mathrm{U}$ & $11 U$ & $9.2 \mathrm{U}$ & 59 & 15 & 150 & 200 \\
\hline STA-G10-R2 & $1.35 \mathrm{U}$ & 0.24 & $0.51 \mathrm{U}$ & $0.41 \mathrm{U}$ & 0.84 & $0.24 U$ & 0.92 & 1.11 \\
\hline
\end{tabular}

\begin{tabular}{|c|c|c|c|c|c|c|c|}
\hline$\omega$ & $\begin{array}{l}\text { Benzo(a)- } \\
\text { Anthracene }\end{array}$ & Chrysene & $\begin{array}{c}\text { Benzo(b)- } \\
\text { Benzo(k)- } \\
\text { Flouranthene }\end{array}$ & $\begin{array}{l}\text { Benzo(a) - } \\
\text { Pyrene }\end{array}$ & $\begin{array}{c}\text { Indeno- } \\
(1,2,3-\mathrm{cd}) \\
\text { Pyrene } \\
\end{array}$ & $\begin{array}{l}\text { Dibenz }(a, h)- \\
\text { Anthracene }\end{array}$ & $\begin{array}{c}\text { Benzo(ghi) } \\
\text { Perylene }\end{array}$ \\
\hline & & & & & & & \\
\hline$\infty$ STA-E8-R2 REP $1^{*}$ & 90 & 77 & 220 & 140 & 100 & 15 & 160 \\
\hline STA-EB-R2 REP $2^{\star}$ & 88 & 92 & 230 & 150 & 100 & 15 & 160 \\
\hline STA-E4-R2* & 140 & 100 & 250 & 190 & 120 & 19 & 180 \\
\hline STA-E4-R2 RE (1)* & 272 & 227 & 574 & 523 & 338 & 53 & 409 \\
\hline STA-AG-R2* & 71 & 64 & 120 & 92 & 64 & 11 & 98 \\
\hline STA-G10-R2 & 0.6 & 0.4 & 1.34 & 0.81 & 0.72 & $0.19 U$ & 0.98 \\
\hline
\end{tabular}

* indicates analysis performed on diluted sample $(1: 10)$

Difference between analyses is thought to be due to sample non-homogeneity.

(1) Re-analysis of sample performed on 2/1/91. Initial results were thought to be low based on the results of of initial MS/MSD analysis perfomred on this sample. 
IABLE 3.10. (Contd)

Quality Control Results for Sediment PAHs

$\begin{array}{ccccccccc}\begin{array}{c}\text { Naph- } \\ \text { thalene }\end{array} & \begin{array}{c}\text { Acenaph- } \\ \text { thylene }\end{array} & \begin{array}{c}\text { Acenaph- } \\ \text { thene }\end{array} & \text { Flourene } & \begin{array}{c}\text { Phenan- } \\ \text { threne }\end{array} & \begin{array}{c}\text { Anthra- } \\ \text { cene }\end{array} & \begin{array}{c}\text { Flouran- } \\ \text { thene }\end{array} & \text { Pyrene } \\ \text { METHOD BLANK } & 1.4 \mathrm{U} & 0.22 \mathrm{U} & 0.55 \mathrm{U} & 0.44 \mathrm{U} & 0.58 \mathrm{U} & 0.25 \mathrm{U} & 0.36\end{array}$

ANALYTICAL DUPLICATE RESULTS :

\begin{tabular}{|c|c|c|c|c|c|c|c|c|c|}
\hline STA-E8-R2 REP 1 & & $44 \mathrm{U}$ & $6.6 \mathrm{U}$ & $17 \mathrm{U}$ & $13 \mathrm{U}$ & 63 & 10 & 220 & 290 \\
\hline STA-E8-R2 REP 2 & & $44 \mathrm{U}$ & $6.7 \mathrm{U}$ & $17 \mathrm{U}$ & $13 \mathrm{U}$ & 58 & 10 & 210 & 280 \\
\hline RPD : & ERR & $0 \%$ & $2 \%$ & $0 \%$ & $0 \%$ & $8 \%$ & $0 \%$ & $5 \%$ & $4 \%$ \\
\hline I-STAT: & ERR & 0.00 & 0.01 & 0.00 & 0.00 & 0.04 & 0.00 & 0.02 & 0.02 \\
\hline
\end{tabular}

iv

\begin{tabular}{|c|c|c|c|c|c|c|c|c|}
\hline & & $\begin{array}{l}\text { Benzo(a) - } \\
\text { Anthracene }\end{array}$ & Chrysene & $\begin{array}{l}\text { Benzo(b)- } \\
\text { Benzo(k)- } \\
\text { Flouranthene }\end{array}$ & $\begin{array}{l}\text { Benzo(a) - } \\
\text { Pyrene }\end{array}$ & $\begin{array}{l}\text { Indeno- } \\
(1,2,3-c d) \\
\text { Pyrene } \\
\end{array}$ & $\begin{array}{l}\text { Dibenz }(a, h)- \\
\text { Anthracene }\end{array}$ & $\begin{array}{l}\text { Benzo(ghi)- } \\
\text { Perylene }\end{array}$ \\
\hline STA-E8-R2 REP 1 & & $0.25 \mathrm{U}$ & $0.22 \mathrm{U}$ & $0.27 \mathrm{U}$ & $0.22 \mathrm{U}$ & $0.35 \mathrm{U}$ & $0.20 \mathrm{U}$ & $0.2 \mathrm{U}$ \\
\hline STA-E8-R2 REP 2 & & 90 & 77 & 220 & 140 & 100 & 15 & 160 \\
\hline $\begin{array}{l}\text { RPD: } \\
\text { I-STAT : }\end{array}$ & $\begin{array}{l}\text { ERR } \\
\text { ERR }\end{array}$ & $\begin{array}{l}88 \\
2 \%\end{array}$ & $\begin{array}{l}92 \\
18 \%\end{array}$ & $\begin{array}{r}230 \\
4 \%\end{array}$ & $\begin{array}{c}150 \\
7 \%\end{array}$ & $\begin{array}{c}100 \\
0 \%\end{array}$ & $\begin{array}{l}15 \\
0 \%\end{array}$ & $\begin{array}{l}160 \\
0 \%\end{array}$ \\
\hline & & 0.01 & 0.09 & 0.02 & 0.03 & 0.00 & 0.00 & 0.00 \\
\hline
\end{tabular}


TABLE 3.10. (contd)

SAMPLE SPIKED:SASTA-G10-R2

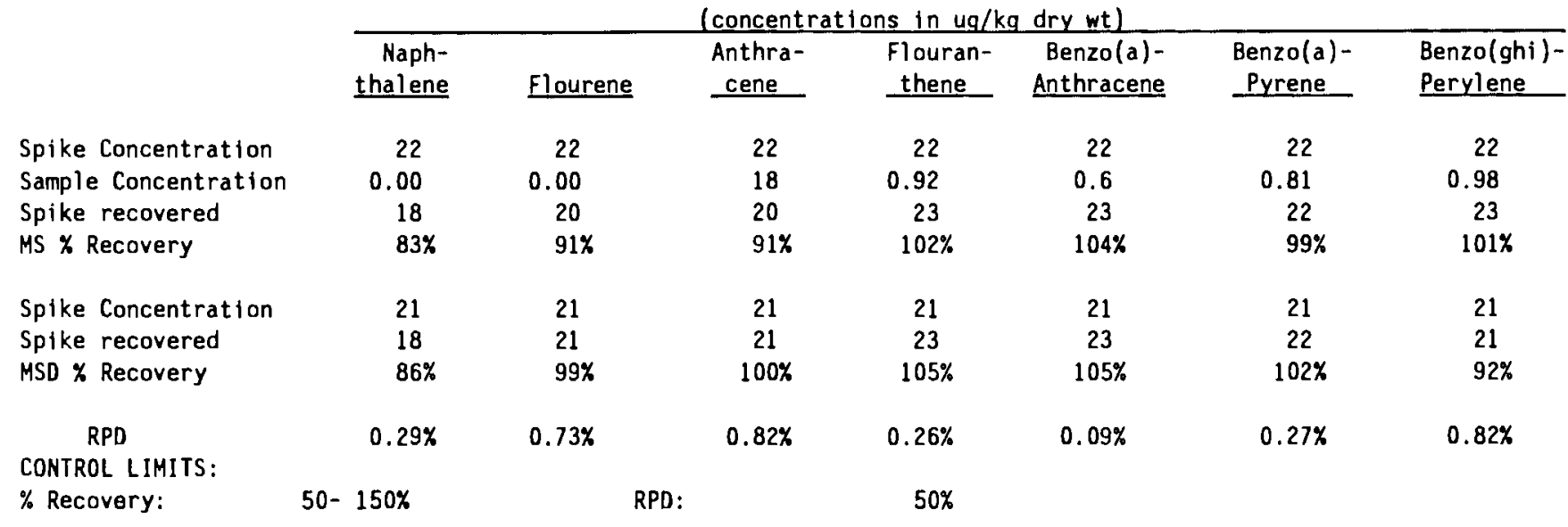

$\omega$

O STANDARD REFERENCE MATERIAL :

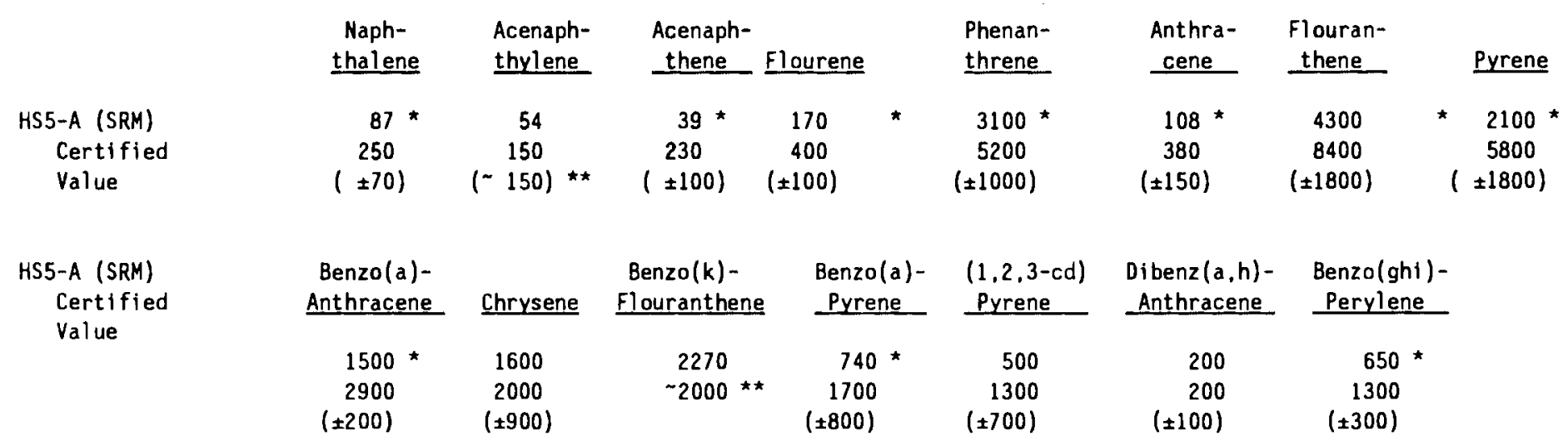

* indicates value out of certified range. Note; SRM was diluted 10-fold to bring into calibration range, resulting in decreased accuracy.

** indicates no certified range available for compound: value given is an estimate. 
IABLE 3.10. (contd)

SURROGATE RECOVERY RESULTS

Sample
Name

STA-E8-R2 Rep 1

STA-E8-R2- rep 2

STA-E4-R2

STA-E4-R2 RE

STA-A6-R2

STA-G10-R2

METHOD BLANK

D10-Flourene

$78 \%$

$88 \%$

$61 \%$

$81 \%$

$78 \%$

$87 \%$

$100 \%$
\% Surrogate Recovery

D10-Anthracene

$58 \%$

$71 \%$

$50 \%$

$98 \%$

$67 \%$

93\%

$80 \%$
D-10 Pyrene

$107 \%$

$117 \%$

$80 \%$

$105 \%$

$80 \%$

$99 \%$

$101 \%$ 
IABLE 3.11. Sediment Polychlorinated Biphenyls (concentrations in ug/kg dry wt.)

\begin{tabular}{|c|c|c|c|c|c|c|c|c|c|}
\hline $\begin{array}{l}\text { Sample } \\
\text { Name }\end{array}$ & $\begin{array}{c}\text { Aroclor- } \\
1016 \\
\end{array}$ & $\begin{array}{c}\text { Aroclor- } \\
1221 \\
\end{array}$ & $\begin{array}{c}\text { Aroclor- } \\
1232 \\
\end{array}$ & $\begin{array}{l}\text { Arcolor- } \\
1242 \\
\end{array}$ & $\begin{array}{l}\text { Aroclor- } \\
1248 \\
\end{array}$ & $\begin{array}{c}\text { Aroclor } \\
1248 \\
\end{array}$ & $\begin{array}{l}\text { Aroclor- } \\
1254 \\
\end{array}$ & $\begin{array}{c}\text { Aroclor- } \\
1260 \\
\end{array}$ & $\begin{array}{l}\text { Surrogate Percent } \\
\text { Dibutylchlorendate }\end{array}$ \\
\hline TARGET DETECTION LIMITS: & $20 \mathrm{ug} / \mathrm{kg}$ & & & & & & & & \\
\hline STA-E8-R2 REP 1 & $44 \mathrm{U}$ & $44 \mathrm{U}$ & $44 U$ & $44 \mathrm{U}$ & $44 \mathrm{U}$ & $44 U$ & $44 \mathrm{U}$ & $44 \mathrm{U}$ & $122 \%$ \\
\hline STA-E8-R2 REP 1 & $46 U$ & $46 U$ & $46 \mathrm{U}$ & $46 U$ & $46 U$ & $46 U$ & $46 U$ & $46 U$ & $115 \%$ \\
\hline STA-E4-R2 & $38 \mathrm{U}$ & $38 \mathrm{U}$ & $38 \mathrm{U}$ & $38 \mathrm{U}$ & $38 \mathrm{U}$ & $38 \mathrm{U}$ & $38 \mathrm{U}$ & $38 \mathrm{U}$ & $90 \%$ \\
\hline STA-AG-R2 & $24 U$ & $24 \mathrm{U}$ & $24 U$ & $24 \mathrm{U}$ & $24 U$ & $24 \mathrm{U}$ & $24 \mathrm{U}$ & $24 U$ & $84 \%$ \\
\hline STA-G10-R2 & $46 U$ & $46 \mathrm{U}$ & $46 \mathrm{U}$ & $46 \mathrm{U}$ & $46 U$ & $46 \mathrm{U}$ & $46 \mathrm{U}$ & $46 U$ & $95 \%$ \\
\hline \multicolumn{10}{|c|}{$U$ indicates parameter not detected at detection limit shown } \\
\hline \multicolumn{10}{|c|}{ Quality Control Results for PCBs in Sediment } \\
\hline METHOD BLANK: & $20 \mathrm{U}$ & $20 \mathrm{U}$ & $20 \mathrm{U}$ & $20 \mathrm{U}$ & $20 \mathrm{U}$ & $20 \mathrm{U}$ & $20 \mathrm{U}$ & $20 \mathrm{U}$ & \\
\hline
\end{tabular}

$\omega$ MATRIX SPIKE RECOVERY RESULT

N Sample Spiked: STA-G1O-R2

\begin{tabular}{|c|c|c|c|c|}
\hline & PCB 1260 & $\begin{array}{c}\text { Surrogate } \\
\text { \% DBC } \\
\end{array}$ & & \\
\hline Spike Concentration & 388 & & & \\
\hline Amount Recovered & 275 & & & \\
\hline Percent Recovery & $71 \%$ & $80 \%$ & & \\
\hline Spike Duplicate Concentration & 394 & & & \\
\hline Amount Recovered & 303 & & & \\
\hline Percent Recovery & $77 \%$ & $84 \%$ & & \\
\hline RPD: & ERR & & & \\
\hline STANDARD REFERENCE MATERIAL & $\begin{array}{l}\text { Aroclor- } \\
1254\end{array}$ & & & $\begin{array}{c}\text { Aroclor- } \\
1254\end{array}$ \\
\hline HS-2 $\operatorname{rep} 1$ & 61 & & $H S-1$ & 23 \\
\hline HS-2 rep 2 & 82 & & & \\
\hline HS-1 Certified Value: & $111.8 \pm 2.5$ & & & $21.8 \pm 1.1$ \\
\hline
\end{tabular}


TABLE 3.12. Sediment Chlorinated Pesticides (concentrations in ug/kg dry wt)

\begin{tabular}{|c|c|c|c|c|c|c|c|c|c|c|c|}
\hline Sample Name & Aldrin & $\underline{A-B H C}$ & B-BHC & D-BHC & Chlordane & $\underline{4} 4^{\prime}$ DDD & $4,4^{\prime} \mathrm{DDE}$ & $4.4^{\prime} D D T$ & Dieldrin & $\begin{array}{c}\text { Endosulfan } \\
\mathrm{I}\end{array}$ & $\begin{array}{c}\text { Endosulfan } \\
\text { II }\end{array}$ \\
\hline TARGET DETECTION LIMITS: & & $\mathrm{ug} / \mathrm{kg}$ & & & & & & & & & \\
\hline STA-E8-R2 & $4.4 \mathrm{U}$ & $4.4 U$ & $4.4 \mathrm{U}$ & $4.4 \mathrm{U}$ & $4.4 \mathrm{U}$ & $4.4 \mathrm{U}$ & $4.4 \mathrm{U}$ & $4.4 \mathrm{U}$ & $4.4 \mathrm{U}$ & $4.4 \mathrm{U}$ & $4.4 U$ \\
\hline STA-E8-R2 & $4.6 \mathrm{U}$ & $4.6 \mathrm{U}$ & $4.6 \mathrm{U}$ & $4.6 \mathrm{U}$ & $4.6 \mathrm{U}$ & $4.6 \mathrm{U}$ & $4.6 \mathrm{U}$ & $4.6 \mathrm{U}$ & $4.6 \mathrm{U}$ & $4.6 \mathrm{U}$ & $4.6 \mathrm{U}$ \\
\hline STA-E4-R2 & $3.8 \mathrm{U}$ & $3.8 \mathrm{U}$ & $3.8 \mathrm{U}$ & $3.8 \mathrm{U}$ & $3.8 \mathrm{U}$ & $3.8 \mathrm{U}$ & $3.8 \mathrm{U}$ & $3.8 \mathrm{U}$ & $3.8 \mathrm{U}$ & $3.8 \mathrm{U}$ & $3.8 \mathrm{U}$ \\
\hline STA-A6-R2 & $3.0 \mathrm{U}$ & $3.0 \mathrm{U}$ & $3.0 \mathrm{U}$ & $3.0 \mathrm{U}$ & $3.0 \mathrm{U}$ & $3.0 \mathrm{U}$ & $3.0 \mathrm{U}$ & $3.0 \mathrm{U}$ & $3.0 \mathrm{U}$ & $3.0 \mathrm{U}$ & $3.0 \mathrm{U}$ \\
\hline STA-G10-R2 & $2.4 \mathrm{U}$ & $2.4 \mathrm{U}$ & $2.4 \mathrm{U}$ & $2.4 \mathrm{U}$ & $2.4 \mathrm{U}$ & $2.4 \mathrm{U}$ & $2.4 \mathrm{U}$ & $2.4 \mathrm{U}$ & $2.4 \mathrm{U}$ & $2.4 \mathrm{U}$ & $2.4 \mathrm{U}$ \\
\hline METHOD BLANK & $2.0 \mathrm{U}$ & $2.0 \mathrm{U}$ & $2.0 \mathrm{U}$ & $2.0 \mathrm{U}$ & $2.0 \mathrm{U}$ & $2.0 \mathrm{U}$ & $2.0 \mathrm{U}$ & $2.0 \mathrm{U}$ & $2.0 \mathrm{U}$ & $2.0 \mathrm{U}$ & $2.0 \mathrm{U}$ \\
\hline & $\begin{array}{c}\text { Endosulfan } \\
\text { Sulfate } \\
\end{array}$ & Endrin & $\begin{array}{c}\text { Endrin } \\
\text { Aldehyde }\end{array}$ & Heptachlor & $\begin{array}{l}\text { Heptachlor } \\
\text { Epoxide }\end{array}$ & $\begin{array}{l}\text { Lindane } \\
(G-B H C)\end{array}$ & Ioxaphene & $\begin{array}{l}\text { Methoxy- } \\
\text { chlor }\end{array}$ & $\begin{array}{l}\text { Endrin } \\
\text { Ketone }\end{array}$ & $\begin{array}{c}\text { Surrogate } \% \\
\text { DBC } \\
\end{array}$ & \\
\hline STA-E8-R2 & $4.4 \mathrm{U}$ & $4.4 \mathrm{U}$ & $4.4 \mathrm{~V}$ & $4.4 \mathrm{U}$ & $4.4 \mathrm{U}$ & $4.4 \mathrm{U}$ & $44.0 \mathrm{U}$ & $22.0 \mathrm{U}$ & $2.0 \mathrm{U}$ & $122 \%$ & \\
\hline STA-E8-R2 & $4.6 \mathrm{U}$ & $4.6 \mathrm{U}$ & $4.6 \mathrm{U}$ & $4.6 \mathrm{U}$ & $4.6 \mathrm{U}$ & $4.6 \mathrm{U}$ & $46.0 \mathrm{U}$ & $23.0 \mathrm{U}$ & $2.0 \mathrm{U}$ & $115 \%$ & \\
\hline STA-E4-R2 & $3.8 \mathrm{U}$ & $3.8 \mathrm{U}$ & $3.8 \mathrm{U}$ & $3.8 \mathrm{U}$ & $3.8 \mathrm{U}$ & $3.8 \mathrm{U}$ & $38.0 \mathrm{U}$ & $19.0 \mathrm{U}$ & $2.0 \mathrm{U}$ & $90 \%$ & \\
\hline STA-A6-R2 & $3.0 \mathrm{U}$ & $3.0 \mathrm{U}$ & $3.0 \mathrm{~V}$ & $3.0 \mathrm{U}$ & $3.0 \mathrm{U}$ & $3.0 \mathrm{U}$ & $30.0 \mathrm{U}$ & $15.0 \mathrm{U}$ & $2.0 \mathrm{U}$ & $84 \%$ & \\
\hline STA-G10-R2 & $2.4 \mathrm{U}$ & $2.4 \mathrm{U}$ & $2.4 \mathrm{U}$ & $2.4 \mathrm{U}$ & $2.4 \mathrm{U}$ & $2.4 \mathrm{U}$ & $24.0 \mathrm{U}$ & $12.0 \mathrm{U}$ & $2.0 \mathrm{U}$ & $95 \%$ & \\
\hline METHOD BLANK & $2.0 \mathrm{U}$ & $2.0 \mathrm{U}$ & $2.0 \mathrm{U}$ & $2.0 \mathrm{U}$ & $2.0 \mathrm{U}$ & $2.0 \mathrm{U}$ & $20.0 \mathrm{U}$ & $10.0 \mathrm{U}$ & $2.0 \mathrm{U}$ & $100 \%$ & \\
\hline
\end{tabular}

$U$ indicates parameter not detected at detection limit shown

Quality Control Results for Sediment Pesticides

PESTICIDE MATRIX SPIKE RESULTS

Sample Spiked: $\quad$ STA-E8-R2

\begin{tabular}{|c|c|c|c|c|c|c|c|}
\hline \multirow{2}{*}{\multicolumn{8}{|c|}{ Endrin Heptachlor (G-BHC) }} \\
\hline & & & & & & & \\
\hline Amount Spiked & 71.4 & 71.4 & 71.4 & 71.4 & 71.4 & 71.4 & \\
\hline Amount Recovered & 57.1 & 60.2 & 57.7 & 83.2 & 52.6 & 64.3 & \\
\hline Percent Recovery & $80 \%$ & $84 \%$ & $81 \%$ & $116 \%$ & $74 \%$ & $90 \%$ & $70 \%$ \\
\hline \multicolumn{8}{|l|}{ MATRIX SPIKE DUPLICATE } \\
\hline Amount Spiked (ug/L) & 71.4 & 71.4 & 71.4 & 71.4 & 71.4 & 71.4 & \\
\hline Amount Recovered (ug/L & 63.6 & 69.6 & 62.9 & 96 & 60.5 & 70.1 & \\
\hline Percent Recovery & $89 \%$ & $97 \%$ & $88 \%$ & $134 \%$ & $85 \%$ & $98 \%$ & $72 \%$ \\
\hline RPD: & $11 \%$ & $14 \%$ & $8 \%$ & $14 \%$ & $14 \%$ & $9 \%$ & \\
\hline
\end{tabular}


from 74 to 134\%. RPDs between spike duplicates ranged from 8 to 14\%. Matrix spike recoveries for Aroclor 1260 were 71 and $77 \%$. RPD between Aroclor spikes was $8 \%$.

One sediment analytical replicate was analyzed. No PCBs or pesticides were detected above detection limits in either replicate initially. Two SRM samples were analyzed, HS-1 and HS-2. Results for HS-1 $(23 \mu \mathrm{g} / \mathrm{kg})$ were in good agreement with the certified value $(21.8 \pm 1.1 \mu \mathrm{g} / \mathrm{kg})$. Results for both reps of HS-2 were somewhat lower than the certified values, but are considered acceptable.

\subsection{WATER CHEMISTRY}

Results for all water analyses including quality control data are presented in Tables 3.13 through 3.19 .

\subsubsection{Total Suspended Solids, Dissolved Organic Carbon, 0il and Grease, Total Petroleum Hydrocarbons}

The results for conventional parameters, including TSS, DOC, total oil and grease, and total petroleum hydrocarbons, are presented in Table 3.13. Detection limit goals for conventional parameters were met for water samples. The matrix spike recovery for DOC in water was $70 \%$. Because of 1 imited sample volumes, no matrix spikes were analyzed for $0 i 1$ and grease or TPH.

One analytical replicate was performed for DOC and TSS. RPDs were 2.8 and $36.4 \%$, respectively.

\subsubsection{Metals}

Metals results for water samples are presented in Table 3.14. Seawater samples were diluted prior to metals analyses for $\mathrm{Cd}, \mathrm{Hg}$, and $\mathrm{Pb}$ to 1 imit interferences from salt water resulting in increased detection limits. Water samples for $\mathrm{Cu}$ analysis were concentrated prior to analysis using a chelating method to reduce interferences from salt water.

Lead was found in the method blank at $0.11 \mu \mathrm{g} / \mathrm{L}$, which is less than five times any of the values detected. Analytical replicate results were in good agreement with RPDs, ranging from 0 to $28 \%$. Analyses of SRMs resulted in values within the certified range for all metals. 


\section{TABLE 3.13. Water Conventionals}

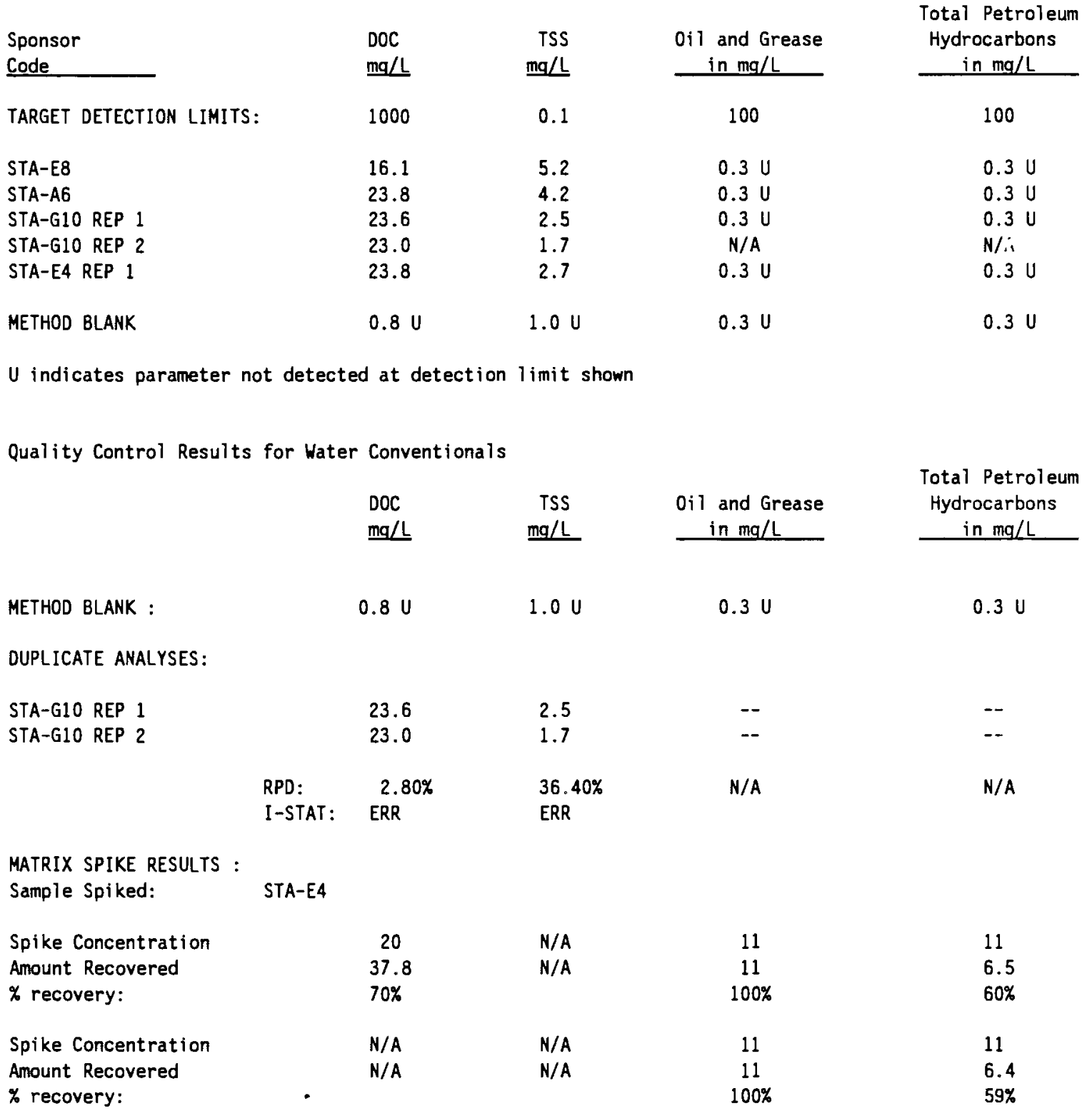




\section{TABLE 3.14. Water Metals}

Sponsor

Code

TARGET DETECTION LIMITS:

$\begin{array}{ll}\text { STA-E8 } & 1 \\ \text { STA-A6 } & 1 \\ \text { STA-G10 } & 1 \\ \text { STA-E4 Rep 1 } & 1 \\ \text { STA-E4 Rep 2 } & 1\end{array}$

* Target Detection Limits not specified.

$U$ indicates parameter not detected at detection limit shown

Quality Control Results for Vater Metals

\begin{tabular}{lll}
\multicolumn{4}{c}{ (concentrations in $u g / L$ ) } \\
\hline Cd & $\mathrm{Cu}$ & $\mathrm{Pb}$
\end{tabular}

$\begin{array}{lllll}1 U & 3.15 & 0.00276 & 0.9 \\ 1 & U & 3.58 & 0.00283 & 1.9 \\ 1 U & 3.55 & 0.00495 & 1.06 \\ 1 U & 3.87 & 0.00401 & 1.16 \\ 1 U & 4.01 & 0.00396 & 1.55\end{array}$

PROCEDURAL BLANK:

Cd

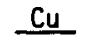

$1 \mathrm{U}$

$0.1 \mathrm{U}$

$\mathrm{Hg}$

$\underline{\mathrm{Pb}}$

ANALYTICAL REPLICATES:

STA-E4 Rep 1

STA-E4 Rep 2

$\begin{array}{lc}1 U & 3.9 \mathrm{U} \\ 1 \mathrm{U} & 4.0 \mathrm{U} \\ 0 \% & 4 \% \\ 0 \% & 2 \%\end{array}$

0.00401

0.00396

$1 \%$

$1 \%$

1.16

1.55

$29 \%$

$14 \%$

STANDARO REFERENCE MATERIAL:

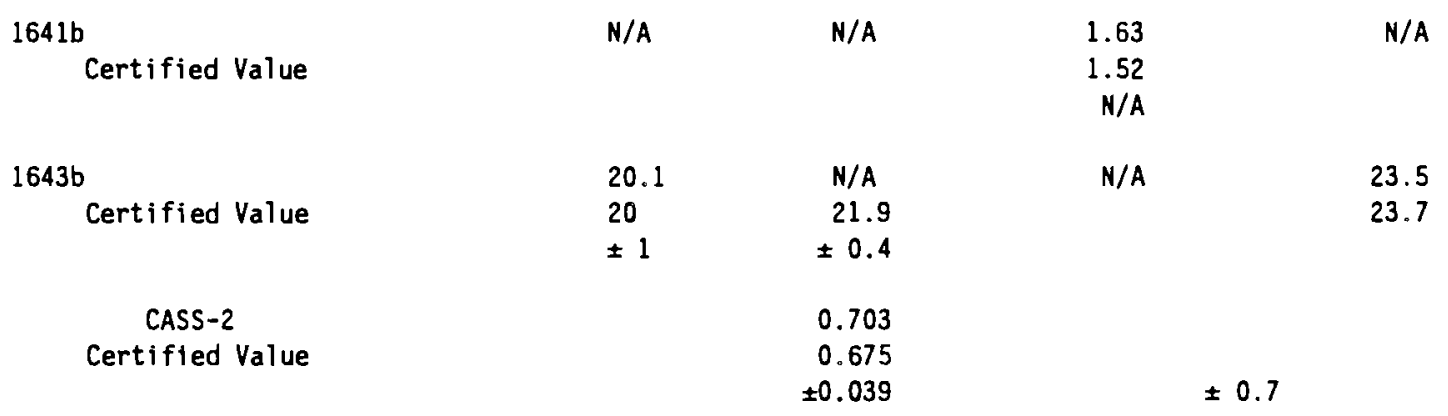

* Outside of certified value range

N/A indicates either not applicable or data not available 


\subsubsection{Butyltins}

Butyltin results for water are presented in Table 3.15. Detection limit goals for butyltins were met. The method blank contained tri-, di- and monobutyltin at concentrations ranging from $8.8 \mathrm{ng} / \mathrm{L}$ for tributyltin to 4.9 $\mathrm{ng} / \mathrm{L}$ for $\mathrm{di}$ - and monobutyltin. The concentrations detected in the samples were similar to those found in the blank with the exception of monobutyltin in four of the five samples analyzed. This could be a result of contamination of monobutyltin from the sample collection vessels. It has been documented that certain glassware may have been treated with monobutyltin during manufacturing (Uhler and Steinhauer 1988).

Two surrogate compounds, tripropyl- and tripentyltin, were used to determine recoveries. The tripentlytin has been found recently to more accurately reflect the behavior of the butyltins. Recoveries for the pentyltins were good, ranging from 74 to $91 \%$. Surrogate recoveries for tripropyltin are lower and are provided for historical reasons only. Matrix spike recoveries for tri- and dibutyltin were good, ranging from 76 to $78 \%$. Agreement between replicate analyses was generally good. RPD values for tetra and tributyltin were 0 and $9 \%$, respectively. RPDs for di- and monobutyltin were somewhat higher (32 and 41\%); however, the concentrations of the compounds measured were relatively low, resulting in exaggerated percent differences.

\subsubsection{Total Phenols, Phenols, and Substituted Phenols}

Phenol results for water are presented in Table 3.16. Detection limit goals were not met for phenols in water. In general, detection limits were a factor of 10 higher than requested. This is because of the nature of the method chosen for analyses of phenols. The detection limit goals for total phenol were too low for the method required for this analysis. The method chosen for measuring phenol and specific substituted phenols was chosen to limit the amount of water needed for analyses since a single extraction was performed for both phenols and PAHs. Insufficient water was available for both a GC/MS extraction for PAH compounds and a GC/FID and GC/ECD extraction for phenolic compounds. 
TABLE 3.15. Water Butyltins

concentrations in $\mathrm{ng} / \mathrm{L}$

Sponsor

Code

TARGET DETECTION LIMITS:

STA-E8

STA-A6

STA-G10 rep 1

STA-G10 rep 2

STA-E4

\begin{tabular}{|c|c|c|c|c|c|}
\hline \multirow{2}{*}{$\begin{array}{l}\text { Tetrabutyl- } \\
\text { tin } \\
\end{array}$} & \multirow{2}{*}{$\begin{array}{l}\text { Tributyl- } \\
\text { tin } \\
\end{array}$} & \multirow{2}{*}{$\begin{array}{l}\text { Dibutyl- } \\
\text { tin } \\
\end{array}$} & \multirow{2}{*}{$\begin{array}{l}\text { Monobutyl- } \\
\text { tin } \\
\end{array}$} & \multicolumn{2}{|c|}{$\begin{array}{c}\text { Surrogate } \\
\text { Recoveries (1) }\end{array}$} \\
\hline & & & & Iripentyltin & Tripropyltin \\
\hline 10 & 10 & 10 & 10 & $40-$ & \\
\hline $2.9 \mathrm{U}$ & $10.1 \mathrm{~B}$ & $3.4 \mathrm{~B}$ & $7.5 \mathrm{~B}$ & $74 \%$ & $56 \%$ \\
\hline $2.6 \mathrm{U}$ & $10.1 \mathrm{~B}$ & $4.7 B$ & $7.4 \mathrm{~B}$ & $83 \%$ & $47 \%$ \\
\hline $2.8 \mathrm{U}$ & $8.5 \mathrm{~B}$ & $5.4 \mathrm{~B}$ & $12.5 \mathrm{~B}$ & $75 \%$ & $27 \%$ \\
\hline $2.3 \mathrm{~V}$ & $9.3 \mathrm{~B}$ & $3.9 \mathrm{~B}$ & $18.9 \mathrm{~B}$ & $74 \%$ & $47 \%$ \\
\hline $2.5 \mathrm{U}$ & $9.6 \mathrm{~B}$ & $2.7 \mathrm{~B}$ & $2.3 \mathrm{~B}$ & $91 \%$ & $39 \%$ \\
\hline
\end{tabular}

$B$ indicates parameter detected in method blank.

$U$ indicates parameter not detected at detection 1 imit shown

(1) Pentyltin is the currently accepted surrogate. Propyltin is the old

surrogate compound which has been found to inadequately reflect tributyltin recoveries.

Data for propyltin are presented for comparison only.

Quality Control Results for Hater Butyltins

concentrations in $\mathrm{ng} / \mathrm{L}$

\begin{tabular}{cccc}
\hline & concentrations in $n g / L$ & Surrogate \\
$\begin{array}{c}\text { Tetrabutyl- } \\
\text { tin }\end{array}$ & $\begin{array}{c}\text { Tributyl- Dibutyl- } \\
\text { tin }\end{array}$ & $\begin{array}{c}\text { Monobutyl- } \\
\text { tin }\end{array}$ & tin \\
\hline
\end{tabular}

METHOD BLANK

$2 \mathrm{U}$

8.8

4.9

4.9

$114 \%$

$49 \%$

REPLICATE ANALYSES

$\begin{array}{lccccc}\text { STA-G10 rep } 1 & & 2.8 U & 8.5 & 5.4 & 12.5 \\ \text { STA-G10 rep } 2 & & 2.3 \mathrm{U} & 9.3 & 3.9 & 18.9 \\ & \text { RPD: } & 0 \% & \text { ERR } & \text { ERR } & \text { ERR }\end{array}$

SPIKE BLANK

\% recovery:

$\begin{array}{lcrr}\text { NS } & 161.7 & 160.5 & \text { NS } \\ - & 76 \% & 78 \% & -\end{array}$

$58 \%$

$57 \%$ 


\section{TABLE 3.16. Water Phenols}

$$
\begin{aligned}
& \text { Sample Location: } \\
& \text { TCT Code: }
\end{aligned}
$$

TOTAL PHENOLS (1)

CHLORINATED PHENOLS (2)

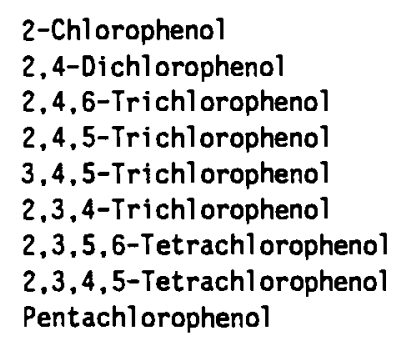

PHENOLS (2)

Target
Detection

Limits

$20 \mathrm{ug} / \mathrm{L}$

$2-10 \mathrm{ug} / \mathrm{L}$

\begin{tabular}{llll} 
& \multicolumn{4}{c}{ (concentrations in $u q / L, p p b)$} \\
\hline STA-E8 & STA-A6 & STA-G10 & STA-E4 \\
$259-17$ & $\underline{259-18}$ & $\underline{259-19}$ & 259-20 Method Blank
\end{tabular}

11

11

11

11

$10 \mathrm{U}$

$\begin{array}{rrrrr}29 U & 28 U & 26 U & 27 U & 25 U \\ 29 U & 28 U & 26 U & 27 U & 25 U \\ 29 U & 28 U & 26 U & 27 U & 25 U \\ 147 U & 139 U & 130 U & 135 U & 125 U \\ 29 U & 28 U & 26 U & 27 U & 25 U \\ 29 U & 28 U & 26 U & 27 U & 25 U \\ 29 U & 28 U & 26 U & 27 U & 25 U \\ 29 U & 28 U & 26 U & 27 U & 25 U \\ 147 U & 139 U & 130 U & 135 U & 125 U\end{array}$

$2-10 \mathrm{ug} / \mathrm{L}$

$\begin{array}{rrlrr}29 U & 28 U & 26 U & 27 U & 25 U \\ 29 U & 28 U & 26 U & 27 U & 25 U \\ 29 U & 28 U & 26 U & 27 U & 25 U \\ 29 U & 28 U & 26 U & 27 U & 25 U \\ 29 U & 28 U & 26 U & 27 U & 25 U \\ 29 U & 28 U & 26 U & 27 U & 25 U \\ 147 U & 139 U & 130 U & 135 U & 125 U\end{array}$

SURROGATE RECOVERIES in percent:

Phenol (D6)

2-Fluorophenol

2,4,6-Tribromophenol

Control Limits *

(10-94)

$(21-100)$

(10-123)
$48 \%$

$51 \%$

$66 \%$
$48 \%$
$48 \%$
$66 \%$

$47 \%$
$49 \%$
$70 \%$

$N / A$

$N / A$ N/A

* EPA CLP CONTROL LIMITS

(1) Total phenols determined by method $510 \mathrm{C}$ (Standard Methods, 1980)

(2) Substituted phenols determined by GC/MS method EPA SW846 8270.

$U$ indicates parameter not detected at detection 1 imit shown

N/A indicates not applicable

Quality Control Results for Vater Phenols

WATER ANALYTICAL REPLICATES:

N/A indicates not applicable

\section{Station}

STA-E8 REP 1

STA-EB REP 2
Total Phenols

$\begin{array}{ll}11 & \text { RPD: } \\ 10 \mathrm{U} & \text { I-STAT }\end{array}$

N/A

$N / A$ 
TABLE 3.16. (contd)

WATER MATRIX SPIKE RESULTS:

(spiked sample : STA-EB)

\section{MATRIX SPIKE}

Amount Spiked

Amount Recovered

$x$ recovery:

MATRIX SPIKE DUPLICATE

Anount Spiked

Amount Recovered

X recovery:

N/A

Total Phenols

200
150
$75 \%$

\begin{tabular}{cl} 
GC/MS Phenols \\
\hline $\begin{array}{c}\text { 2- } \\
\text { chloro- } \\
\text { phenol }\end{array}$ & $\begin{array}{l}\text { 3-methyl- } \\
\text { phenol }\end{array}$ 4-nitro- Pentachloro- \\
Phenol phenol
\end{tabular}

535

535
257
$48 \%$

$\begin{array}{ll}535 & 535 \\ 321 & 374 \\ 60 \% & 70 \%\end{array}$

$60 \% \quad 70 \%$

$\begin{array}{ll}535 & 535 \\ 321 & 562\end{array}$

$60 \% \quad 105 \%$

$\begin{array}{ccccl}526 & 526 & 526 & 526 & 526 \\ 210 & 310 & 342 & 281 & 526 \\ 40 \% & 60 \% & 65 \% & 54 \% & 100 \%\end{array}$


All acid surrogate recoveries were within the EPA control limits. Matrix spike and matrix spike duplicate recoveries were acceptable, ranging from 40 to $105 \%$.

\subsubsection{Polynuclear Aromatic Hydrocarbons}

Results for PAH analyses in water are presented in Table 3.17. Detection limit goals for PAH compounds were exceeded by a factor of approximately 3 . The detection limit goal for PAHs was $10 \mu \mathrm{g} / \mathrm{L}$, and detection limits attained for this study ranged from 26 to $29 \mu \mathrm{g} / \mathrm{L}$.

No compounds were detected in the method blank. Two base/neutral surrogate recoveries for semivolatiles in water exceeded EPA control limits for 3 of the 4 samples; however, the recoveries were greater than $10 \%$, and no action was taken. Matrix spike and matrix spike duplicate recoveries for acid compounds were acceptable, ranging from 40 to $105 \%$. Base/neutral spiking compounds were inadvertently left out before extraction; however, recoveries for the acid spiking compounds are acceptable.

\subsubsection{Chlorinated Pesticides and Polychlorinated Biphenyls}

Results for PCBs and chlorinated pesticides in water are presented in Tables 3.18 and 3.19 , respectively. Detection limit goals were met for PCBs and pesticides in water samples. No constituents were detected in the method blank. Surrogate recoveries for all samples ranged from 89 to $101 \%$. Matrix spike and matrix spike duplicate recoveries for pesticides were acceptable, ranging from 69 to $114 \%$. RPDs between spike duplicates were also acceptable ranging from 1 to $15 \%$.

No analytical replicates were analyzed because of 1 imited sample volumes, and no SRMs exist for PCBs or pesticides in water. 
TABLE 3.17. Water Polynuclear Aromatic Hydrocarbons

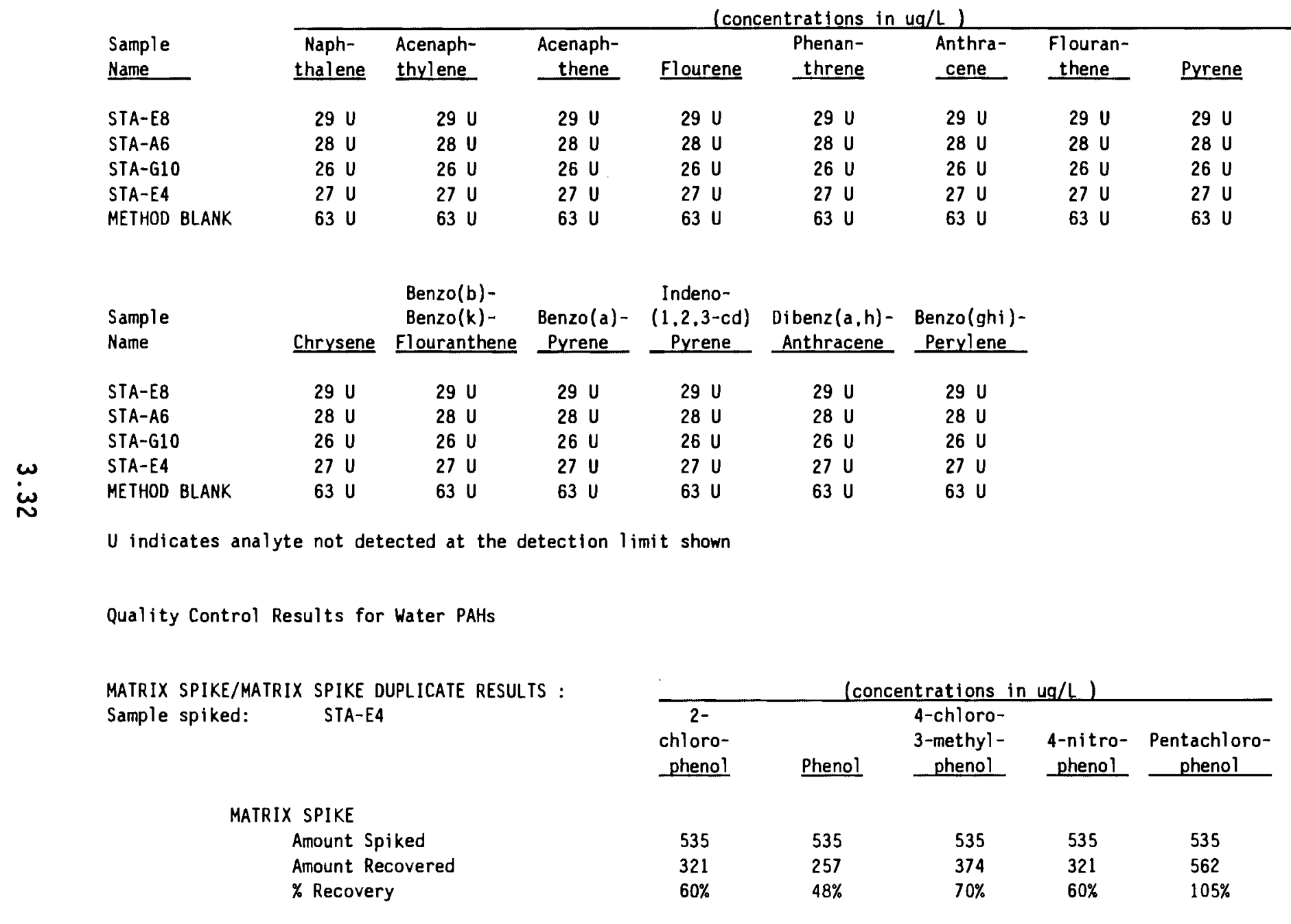




\section{TABLE 3.17. (contd)}

MATRIX SPIKE DUPLICATE

Amount Recovered

\% Recovery

MSD * Recovery

$\begin{array}{lllll}526 & 526 & 526 & 526 & 526 \\ 310 & 210 & 342 & 281 & 526 \\ 59 \% & 40 \% & 65 \% & 53 \% & 100 \%\end{array}$

RPD

ERR

ERR

ERR ERR

ERR

CONTROL LIMITS:

\% Recovery:

50- $150 \%$

RPD:

$50 \%$

PAH SURROGATE RECOVERY RESULTS

Sample
Name

CONTROL LIMITS: (1)

STA-E8

STA-A6

STA-G10

STA-E4

METHOD BLANK

(1) CONTROL LIMITS ESTABLISHED BY EPA CLP.

Nitrobenzene (D5)

(35-114)
* Surrogate Recovery

2-Fluorobi phenyl

(43-116)

$19 \% *$

$22 \% *$

$24 \%$ *

$24 \%$ *

$33 \%^{\star}$
Terphenyl (014)

(33-141)

Surrogate Recovery Exceeds Control Limit 
TABLE 3.18. Water Polychlorinated Biphenyls

\begin{tabular}{|c|c|c|c|c|c|c|c|c|}
\hline \multicolumn{9}{|c|}{ (concentrations in ug/L) } \\
\hline $\begin{array}{l}\text { Sample } \\
\text { Name }\end{array}$ & $\begin{array}{c}\text { Aroclor- } \\
1016 \\
\end{array}$ & $\begin{array}{l}\text { Aroclor- } \\
1221 \\
\end{array}$ & $\begin{array}{c}\text { Aroclor- } \\
1232 \\
\end{array}$ & $\begin{array}{l}\text { Aroclor- } \\
1242 \\
\end{array}$ & $\begin{array}{c}\text { Aroclor- } \\
1248 \\
\end{array}$ & $\begin{array}{c}\text { Aroclor- } \\
1254 \\
\end{array}$ & $\begin{array}{c}\text { Aroclor- } \\
1260 \\
\end{array}$ & $\begin{array}{c}\text { Surrogate } \\
\text { Percent Recovery } \\
\text { DBC } \\
\text { (Dibutylchlorendate) }\end{array}$ \\
\hline TARGET DETECTION LIMITS: & 5 & 5 & 5 & 5 & 5 & 5 & 5 & \\
\hline STA-E8 & $0.6 \mathrm{U}$ & $0.6 \mathrm{U}$ & $0.6 \mathrm{U}$ & $0.6 \mathrm{U}$ & $0.6 \mathrm{U}$ & $0.6 \mathrm{U}$ & $0.6 \mathrm{U}$ & $101 \%$ \\
\hline STA-AG & $0.6 \mathrm{U}$ & $0.6 \mathrm{U}$ & $0.6 \mathrm{U}$ & $0.6 \mathrm{U}$ & $0.6 \mathrm{U}$ & $0.6 \mathrm{U}$ & $0.6 \mathrm{U}$ & $92 \%$ \\
\hline \multirow{2}{*}{$\begin{array}{l}\text { STA-G10 } \\
\text { STA-E4 }\end{array}$} & $0.6 \mathrm{U}$ & $0.6 U$ & $0.6 \mathrm{U}$ & $0.6 \mathrm{U}$ & $0.6 \mathrm{U}$ & $0.6 \mathrm{U}$ & $0.6 \mathrm{U}$ & $89 \%$ \\
\hline & $0.6 \mathrm{U}$ & $0.6 \mathrm{U}$ & $0.6 \mathrm{U}$ & $0.6 \mathrm{U}$ & $0.6 \mathrm{U}$ & $0.6 \mathrm{U}$ & $0.6 \mathrm{U}$ & 89\% \\
\hline METHOD BLANK & $0.6 \mathrm{U}$ & $0.6 \mathrm{U}$ & $0.6 \mathrm{U}$ & $0.6 \mathrm{U}$ & $0.6 \mathrm{U}$ & $0.6 \mathrm{U}$ & $0.6 \mathrm{U}$ & $96 \%$ \\
\hline \multicolumn{9}{|c|}{ indicates parameter not detected at detection 1 imit shown } \\
\hline \multicolumn{9}{|c|}{ Quality Control Results for Water PCBs } \\
\hline \multicolumn{9}{|l|}{ MATRIX SPIKE RESULTS } \\
\hline Sample Sptked: STA- E8 & & Aldrin & $\underline{4,4^{\prime} \mathrm{DDT}}$ & \multirow[t]{2}{*}{ Dfeldrin } & Endrin & \multirow[t]{2}{*}{ Heptachlor } & \multirow[t]{2}{*}{$\begin{array}{l}\text { Lindane } \\
(G-B H C)\end{array}$} & $\begin{array}{c}\text { Surrogate } \\
\text { DBC }\end{array}$ \\
\hline \multicolumn{2}{|l|}{ MATRIX SPIKE } & & & & & & & \\
\hline Amount Spil & g/L) & 1.42 & 1.42 & 1.42 & 1.42 & 1.42 & 1.42 & -- \\
\hline Amount Recc & $(\mathrm{ug} / \mathrm{L})$ & 1.00 & 1.39 & 1.24 & 1.4 & 0.97 & 1.37 & -- \\
\hline Percent Rec & & $70 \%$ & $98 \%$ & $87 \%$ & $98 \%$ & $68 \%$ & $96 \%$ & $82 \%$ \\
\hline MATRIX SPIKE & ATE & & & & & & & \\
\hline Amount $\mathrm{Sp}$ & $u g / L)$ & 1.42 & 1.42 & 1.42 & 1.42 & 1.42 & 1.42 & -- \\
\hline Amount Rec & $(u g / L)$ & 0.97 & 1.52 & 1.38 & 1.6 & 0.97 & 1.55 & -- \\
\hline Percent Re & & $69 \%$ & $108 \%$ & $98 \%$ & $114 \%$ & $69 \%$ & $111 \%$ & $93 \%$ \\
\hline & RPD: & $1 \%$ & $10 \%$ & $12 \%$ & $15 \%$ & $1 \%$ & $14 x$ & $13 \%$ \\
\hline
\end{tabular}


TABLE 19. Water Chlorinated Pesticides

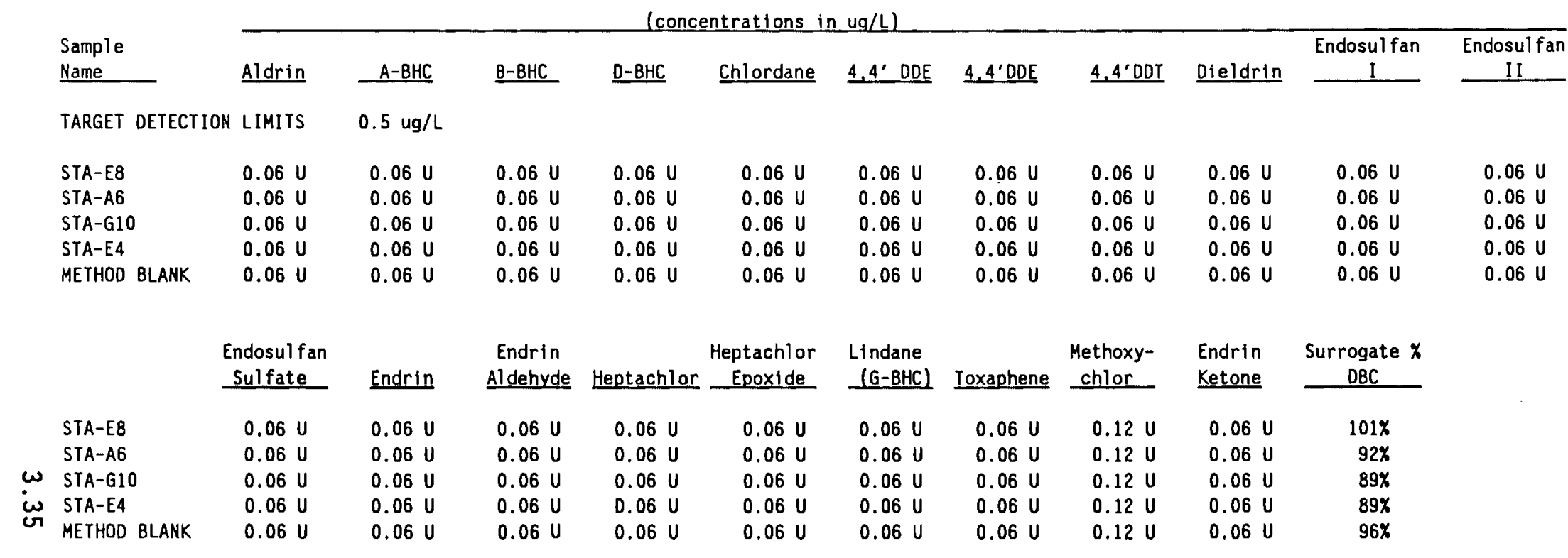

$U$ indicates parameter not detected at detection 1 imit shown

Quality Control Results for Water Pesticides

MATRIX SPIKE RESULTS

\begin{tabular}{|c|c|c|c|c|c|c|c|}
\hline & Alrin & $4,4^{\prime}$ DDT & Dieldrin & Endrin & Heptachlor & $\begin{array}{l}\text { Lindane } \\
\text { (G-BHC) }\end{array}$ & $\begin{array}{c}\text { Surrogate } \\
\text { DBC }\end{array}$ \\
\hline \multicolumn{8}{|l|}{ MATRIX SPIKE } \\
\hline Amount Spiked (ug/L) & 1.42 & 1.42 & 1.42 & 1.42 & 1.42 & 1.42 & -- \\
\hline Amount Recovered (ug/L) & 1 & 1.39 & 1.24 & 1.4 & 0.97 & 1.37 & -- \\
\hline Percent Recovery & $70 \%$ & $98 \%$ & $87 \%$ & $98 \%$ & $68 \%$ & $96 \%$ & $82 \%$ \\
\hline \multicolumn{8}{|l|}{ MATRIX SPIKE DUPLICATE } \\
\hline Amount Spiked (ug/L) & 1.42 & 1.42 & 1.42 & 1.42 & 1.42 & 1.42 & -- \\
\hline Amount Recovered (ug/L) & 0.97 & 1.52 & 1.38 & 1.6 & 0.97 & 1.55 & -- \\
\hline Percent Recovery & $69 \%$ & $108 \%$ & $98 \%$ & $114 \%$ & $69 \%$ & $111 \%$ & $93 \%$ \\
\hline RPD: & $1 \%$ & $10 \%$ & $12 \%$ & $15 \%$ & $200 \%$ & $14 \%$ & $200 \%$ \\
\hline
\end{tabular}




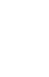
. 


\subsection{REFERENCES}

American Society for Testing and Materials (ASTM). 1972. Standard Method for Particle-Size Analysis of Soils. D422. American Society for Testing and Materials, Philadelphia, Pennsylvania.

American Society for Testing and Materials (ASTM). 1978. Standard Method for Dry Preparation of Soil Samples for Particle-Size Analysis and Determination of Soil Constants. D421. American Society for Testing and Materials, Philadelphia, Pennsylvania.

Bloom, N.S., and E.A. Crecelius. 1983. "Determination of Mercury in Seawater at Sub-Nanogram per Liter Levels." Mar. Chem. 14:49-59.

Bloom, N.S., and E.A. Crecelius. 1984. "Distribution of Silver, Mercury, Lead, Copper and Cadmium in Central Puget Sound Sediments." Mar. Chem. $21: 337-390$.

Nielson, K.K. and R. W. Sanders. 1983. "Multielement Analys is of Unweighed Biological and Geological Samples Using Backscatter and Fundamental

Parameters." Adv. X-Ray Analy. 26:385-390.

Puget Sound Estuary Program (PSEP). 1986. Recommended Protocols for Measuring Selected Environmental Variables in Puget Sound. Vols. 1 and 2. Prepared for the Puget Sound Estuary Program by Tetra-Tech, Inc, Bellevue, Washington.

Standard Methods. 1980. Standard Methods for the Examination of Water and Wastewater. 15th Edition. American Public Health Association (APHA), American Water Works Association (AWWA), Water Pollution Control Federation (WPCF). Washington, D.C.

Uhler, A.D., and W.G. Steinhauer. 1988. Measurement of Butylt in Species in Water by $n$-Pertyl Derivatization with GC/FPD Detection. Battelle 0cean Sciences, Duxbury, Massachusetts.

Unger, M. A., W. G. Macintyre, J. Greaves, and R. J. Huggett. 1986. "GC Determination of Butyltin in Natural Water by Gas Chromatography/Flame Photoionization Spectrometry of Hexyl Derivatives with Mass Spectrometry Confirmation". Chemosphere 15(4):461-470.

U.S. Environmental Protection Agency (EPA). 1979. Methods for Chemical Analysis of Water and Wastes. EPA-600/4-79-020, Method 413.2 and 418.1, U.S. Environmental Protection Agency, Washington, D.C.

U.S. Environmental Protection Agency (EPA). 1986. Test Methods for Evaluating Solid Waste: Physical/Chemical Methods. SW-846. U.S. Document No. 955-00100000, U.S. Environmental Protection Agency, Washington, D.C. 
. 
APPENDIX A

THE QUALITY ASSURANCE PLAN 


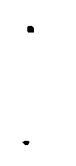


QUALITY ASSURANCE PLAN FOR THE

ANALYZING SEDIMENT, WATER AND BIOLOGICAL SAMPLES FROM THE BAY FARM BORROW AREA PROJECT

Phit ham

RM Thom, Project Manager (Concurrence) $\frac{12 \text { tale } 91}{\text { Date }}$

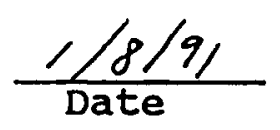

$\frac{4 / 2 / 91}{\text { Date }}$
2 seb

R. Cuello, Quality Engineer (Concurrence)

ent Ence

R.M. Ecker, Manager Marine Sciences Laboratory (Approval) 
PROJECT IMPACT LEVEL III

\subsection{INTRODUCTION}

\subsection{Title:}

QA Plan for the Analyzing Sediment, Water and Biological Samples from the Bay Farm Borrow Area project.

\subsection{Client:}

U.S. Army Corps of Engineers (USACE), San Francisco District

\subsection{QA Program/Organization:}

This Quality Assurance Plan (QAP) includes the appropriate elements (sections) from the PNL Quality Assurance Manual, PNL-MA-70, Part II, Good Practices Standard (GPS) and applicable client requirements.

In addition to the quality assurance requirements specified in this Quality Assurance Plan (QAP) and unless specifically exempted, project personnel shall follow the protocols of the PNL-MA-70 Quality Assurance Program, Part II, the Good Practices Standard (GPS). The Good Practices Standard establishes quality assurance requirements for work performed by project personnel on approved Impact Level III projects. The quality assurance requirements contained in the Good Practices Standard are grouped into four (4) major areas:

- Management and Administration,

- Support Activities,

- Work Performance, and

- Information and Records Management.

The Management and Administration Section of the GPS provides QA requirements and guidelines for proposal preparation, the development of work plans, technical reviews, staff selection and training, and management effectiveness assessments of the implemented quality assurance measures.

The Support Activities Section of the GPS provides QA requirements and guidelines for activities which require the use of support staff. Items covered in this section include procurements and subcontracts, chemical/physical analytical services, engineering/design services and facilities services.

The Work Performance Section of the GPS provides QA requirements and guidelines for project personnel who will perform analytical studies, experimental studies, hardware systems development and software preparation and control activities.

The Information and Records Management Section of the GPS provides QA requirements and guidelines for the generation, maintenance and management of 
project records and files. Requirements regarding the use and control of Laboratory Record Books (LRBs) are delineated in PNL Act Now Directive 89-1: Use of Laboratory Record Books.

The project organization with key personnel identified is located in Exhibit 3.1 Project Interfaces.

\subsection{PROJECT DESCRIPTION}

This QA Plan is intended to address all of the various types of activities that the Bay Farm Borrow Area project might potentially perform. A detailed description of each specific activity shall be included in the Scope of Services/Statement of Work.

\section{1 objectives}

The objective of this project is to perform sediment, water and biological sample analysis. The analysis results will assist in determining whether the site is suitable for open-water disposal pursuant to the requirements of Section 404 of the Clean Water Act.

\subsection{PROJECT ORGANIZATION AND RESPONSIBILITIES}

Line authority, quality assurance authority and support with PNL and interfaces with San Francisco Army Corps of Engineers (USACE) are shown in Exhibit 3.1. The responsibilities of key PNL personnel are summarized in Section 3.1.

\subsection{Responsibilities of Key Personnel}

Personnel

Marine Sciences

Laboratory Manager

Project Manager

Quality Engineering Group Leader

Quality Engineer

\section{Responsibilities}

Provides management review of project. Assures appropriate and qualified staff are available.

Provides overall direction of the project at PNL and day-to-day activities necessary to accomplish all project objectives. Ensures that the planning documents are prepared and adhered to and that data and reports are produced in a timely manner. Has direct contact with the PNL Quality Engineer and the client.

Provides for independent quality assurance reviews, surveillances, and data quality and traceability audits. Is responsible for reviewing and has sign off authority for QA Plans.

Provides $Q A$ support in such areas as $Q A$ requirement guidance/interpretation, audit preparation, $Q A P 1$ an development or revision, resolving $Q A$ problems and 
document review. Also provides independent oversight to verify project activities are being performed

consistent with applicable requirements identified in this QA PIan.

A. 4 
EXHIBIT 3.1 Project Interfaces

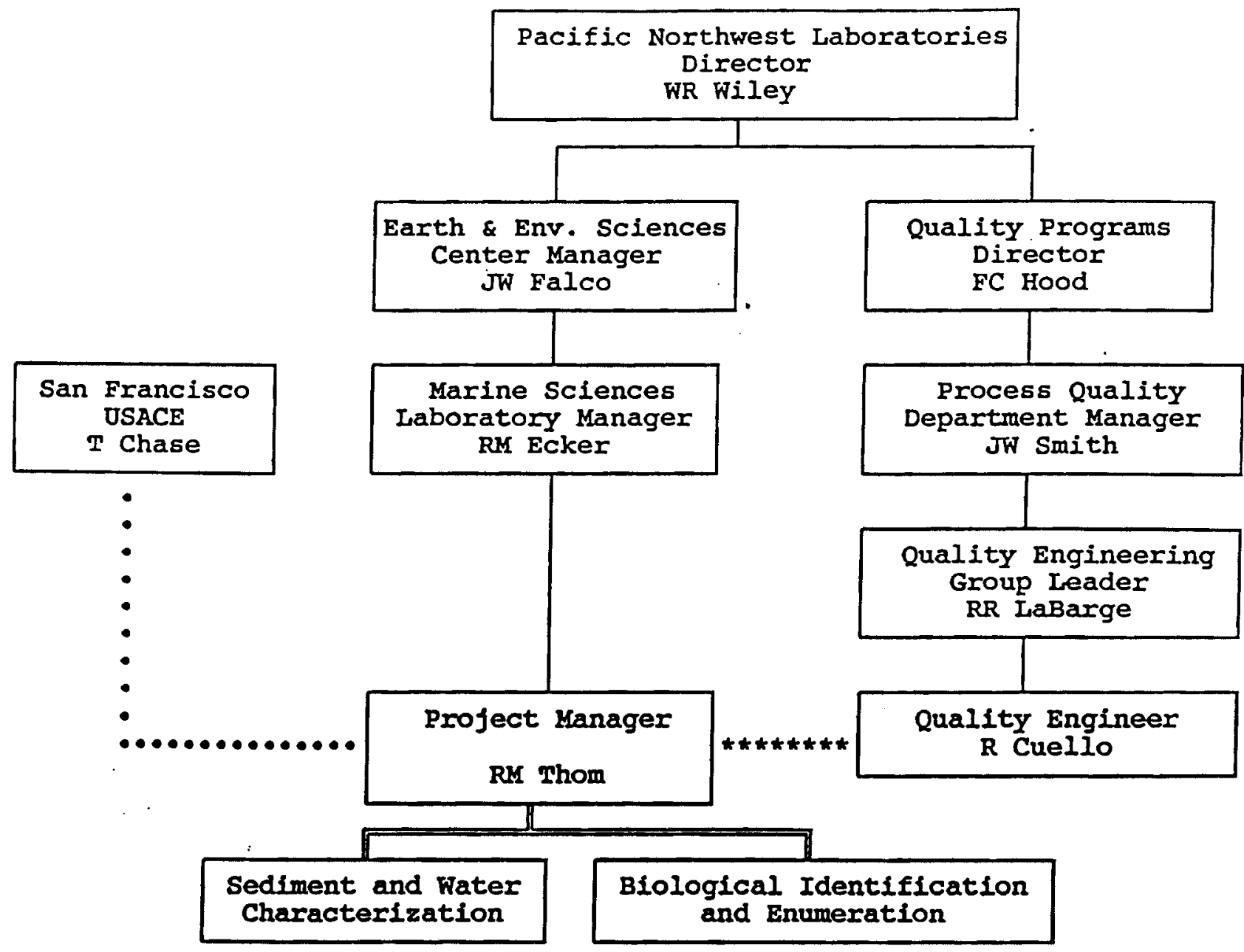

Line Management Guidance

Profect Technical Guidance

Project QA Support

Client Interface 


\subsection{QA OBJECTIVES FOR MEASUREMENT DATA}

The characteristics used to define data quality are accuracy, precision, completeness and detection limits. The definition used for these terms are as follows:

Precision - a measure of mutual agreement among individual measurements of the same property, usually under prescribed similar conditions. Precision can be expressed in terms of the relative percent difference, I-Stat and/or standard deviation.

$$
\begin{aligned}
\mathrm{RPD}= & \frac{\left(C_{1}-C_{2}\right) \times 100 \%}{\left(C_{1}+C_{2}\right) / 2} \\
R P D & =\text { relative percent difference } \\
C_{1} & =\text { larger of the two observed values } \\
C_{2} & =\text { smaller of the two observed values }
\end{aligned}
$$

I-Stat $=\frac{C_{1}-C_{2}}{C_{1}^{1}+C_{2}}$

$C_{1}=$ larger of the two observed values

$C_{2}^{1}=$ smaller of the two observed values

If calculated from three or more replicates, use relative standard deviation (RSD) or coefficient of variation rather than RPD:

$$
\begin{aligned}
\operatorname{RSD}=(s / \bar{x}) \times 100 \% \\
\text { RSD }=\text { relative standard deviation } \\
\frac{s}{\bar{x}}=\text { standard deviation }
\end{aligned}
$$

Standard deviation, $s$, is defined as follows:

$$
\begin{aligned}
s=\sum_{i=1}^{n} \sqrt{\frac{\left(y_{i}-x\right)^{2}}{n-1}} \\
s=\text { standard deviation } \\
y_{i}=\text { measured value of the replicate } \\
x=\text { mean of replicate measurements } \\
n=\text { number of replicates }
\end{aligned}
$$

Accuracy - a measure of the bias in a system.

- For measurements where matrix spikes are used:

$\% R=100 \times \frac{S-U}{C_{\text {sa }}}$ 


$$
\begin{aligned}
\% R & =\text { percent recovery } \\
S & =\text { measured concentration in spiked al iquot } \\
U & =\text { measured concentration in unspiked al iquot } \\
C_{S a} & =\text { actual concentration of spike added }
\end{aligned}
$$

- For situations where a standard reference material (SRM) is used instead of or in addition to matrix spikes:

$$
\begin{aligned}
\% R=100 \times \frac{C_{m}}{C_{\text {srm }}} \\
\% R=\text { percent recovery } \\
C_{m}=\text { measured concentration of SRM } \\
C_{\text {srm }}=\text { actual concentration of SRM }
\end{aligned}
$$

Completeness - a measure of the amount of valid data obtained from a measurement system compared to the amount that was expected to be obtained under correct normal conditions.

Defined as follows for all measurements:

$$
\begin{aligned}
\% C=100 & \times \frac{V}{n} \\
\% C= & \text { percent completeness } \\
V= & \text { number of measurements judged valid } \\
n= & \text { total number of measurements necessary to } \\
& \text { achieve a specified statistical level of } \\
& \text { confidence in decision making }
\end{aligned}
$$

Method Detection Limit

Defined as follows for all measurements:

$$
\begin{aligned}
& M D L=t_{(n-1,1-\alpha=0.99)} \times S \\
& M D L=\text { method detection limit } \\
& t_{(n-1,1-\alpha=0.99)}=\begin{array}{l}
\text { standard deviation of the replicate analyses } \\
\text { confidence level and a standard deviation } \\
\text { estimate with } n-1 \text { degrees of freedom }
\end{array}
\end{aligned}
$$

Data quality objectives (DQOS) for precision, accuracy and target detection limits are shown in Table 4.1. Chemistry accuracy objectives are expressed in terms of an acceptable range of recovery and precision objectives in terms of percent relative precision.

The precision and accuracy objectives specified in Table 4.1 are based on standard method performance information, when available, and historical laboratory performance. For some methods, this information was not available; a - is marked in the summary tables. As additional analytical results are compiled this information will be completed. 
TABLE 4.1 Data Quality Objectives for Chemistry

\section{Data Quality Objectives}

Analyte

\section{Conventionals \\ TOC \\ 0 il and Grease \\ Total PHCs \\ Grain Size \\ DOC \\ TSS}

\section{Metals}

Silver

Arsenic

Cadmium

Chromium

Copper

Mercury

Nickel

Lead

Selenium

Zinc

\section{Organics}

Butyltins

PCBS

PAHs

Pesticides

Phenols

Total Phenol

Detection

Limit

$\begin{array}{llll}\text { Reference } & \text { Range of } & \text { Relative } & \text { Sed. Water } \\ \text { Method } & \text { Recovery } \% & \text { Precision }\end{array}$

Method Recovery \%

Precision $\mathrm{mg} / \mathrm{Kg} \mathrm{mg} / \mathrm{L}$

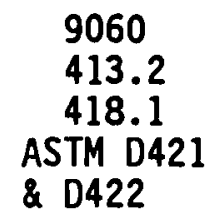

$20 \overline{0} D$

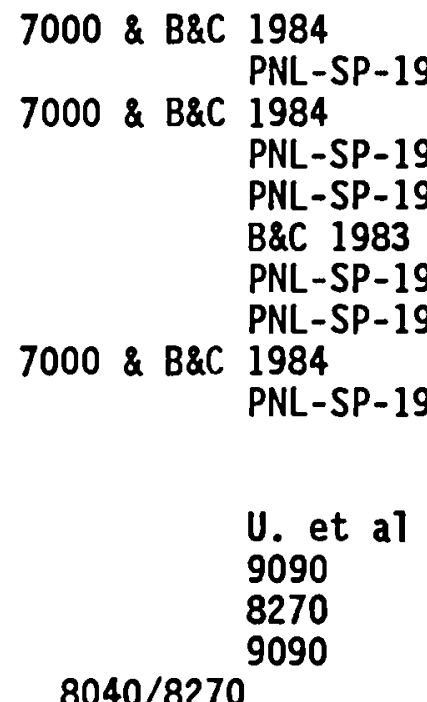

$8040 / 8270$

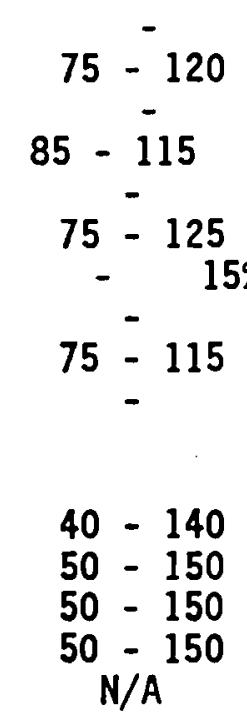

$N / A$

$\begin{array}{cll}10 \% & 0.1 \% & N / A \\ 10 \% & 20 & 100 \\ - & 20 & 100 \\ - & \text { N/A } & \text { N/A } \\ & & \\ - & \text { N/A } & 0.1 \% \\ - & \text { N/A } & 0.1\end{array}$

$\begin{array}{lll}15 \% & 0.1 & \text { N/A } \\ 15 \% & 2 & \text { N/A } \\ 15 \% & 0.1 & 0.05 \\ 15 \% & 2 & \text { N/A } \\ 15 \% & 2 & 0.2 \\ 15 \% & 0.02 & 0.1 \\ \text { N/A } & & \\ 15 \% & 2 & 0.1 \\ 15 \% & 1 & N / A \\ 15 \% & 2 & N / A\end{array}$

$\begin{array}{rll}20 \% & 0.01 & 0.001 \\ 20 \% & 0.02 & 0.005 \\ 20 \% & 0.02 & 0.01 \\ 20 \% & 0.002 & 0.0005 \\ 20 \% & 0.02 & 0.002 \\ & \text { to } & \text { to }\end{array}$

$20 \% \quad \begin{array}{lll}0.1 & 0.01 \\ 0.02 & 0.02\end{array}$ to 0.1 
D421 - ASTM, 1978 - Standard Method for Dry Preparation of Soil Samples for Particle-size Analysis and Determination of Soil Constants

D422 - ASTM, 1972 - Standard Method for Particle-size Analysis of Soils

7000 - EPA, SW846 Series - Atomic Absorption Spectroscopy

8040 - EPA, SW846 Series - Phenols (Sediment)

8270 - EPA, SW846 Series - Polynuclear Aromatic Hydrocarbons

9060 - EPA, SW846 Series - Total Organic Carbon

9090 - EPA, SW846 Series - Organochlorine Pesticides \& PCBS

$U$ et al. Unger et al., 1986. "GC Determination of Butyltins in Natural Waters by Flame Photometric Detection of Hexyl Derivatives with Mass Spectrometric Confirmation." Chemosphere 15(4):461-470

B\&C 1983 Bloom, N.S., and E.A. Crecelius. 1983. "Determination of Mercury in Seawater at Sub-Nanogram per Liter Levels." Marine Chemistry 14:4959.

B\&C 1984 Bloom, N.S., and E.A. Crecelius. 1984. "Distribution of Silver, Mercury, Lead, Copper and Cadmium in Central Puget Sound Sediments." Marine Chemistry 21:377-390.

413.2 \& U.S. Environmental Protection Agency (EPA). 1979. Methods for the 418.1 Evaluation of Water and Wastes. EPA-600-4-79-020 (Methods 413.2 and 418.1). Environmental Monitoring and Support Laboratory, Cincinnati, Ohio.

209 D \& Standard Methods. 1980. Standard Methods for the Examination of

$510 \mathrm{C}$ - Water and Wastewater, 15 th Edition, APHA-AWWA-WPCF.

PNL-SP-19 Sanders, R.W., 1987. Energy Dispersive X-Ray Fluorescence Spectrometry. PNL SOP.

N/A - Not Applicable

- Not Available 


\subsection{SAMPLING AND ANALYSIS}

\subsection{Sample Archiving}

That portion of each individual sediment and water sample remaining after chemical analysis shall be archived at $4^{\circ} \mathrm{C} \pm 2^{\circ} \mathrm{C}$ for a minimum of 30 days after analysis date. Temperature of the archival facility shall be checked and recorded daily with a thermometer calibrated.

\subsection{Changes to the Analys is Schedule}

Permanent planned changes to the analysis schedules must be verbally agreed upon by the PNL project manager and the San Francisco USACE contact for this program. The change must be documented, including as a minimum 1) the effective date of change and 2 ) the reason for the change.

\subsection{Chemical Analysis}

Potential chemical constituents to be analyzed for, as well as the corresponding standard analytical methods on which the primary analytical laboratory bases its procedures are shown in Table 4.1. The Scope of Services/Statement of Work must identify which specific constituents will be analyzed. If there are project specific constituents that aren't included in Table 4.1, they must be identified in future or amended planning documents.

The sediment and water samples shall be analyzed for the parameters (using the detection limits) specified in Table 4.1. If these detection limits are not achievable, attempts shall be made to achieve the lowest practical detection limits. The results shall be reported in both wet weight and dry weight for the sediments.

\subsection{SAMPLE CUSTODY AND FIELD/LAB DOCUMENTATION}

All of the samples shall be handled in such a manner to preclude the contamination of or loss of any of the sampled sediments or waters. The sample containers shall be sealed to prevent any moisture loss and possible contamination, and shall be kept on ice, but not frozen, until delivered to the place of testing.

Following receipt of samples at the Marine Sciences Laboratory, all samples shall be stored at $4^{\circ} \mathrm{C} \pm 2^{\circ} \mathrm{C}$ until disposal. Temperature of the storage facility shall be checked and recorded daily with a calibrated thermometer.

\subsection{Sample Chain-of-Custody}

The chain-of-custody of samples from Sequim to the analytical labs shall be controlled in accordance with MSL-5, Sample Chain-of-Custody. The chain-ofcustody form that shall be used is shown in MSL-5.

Only black ink shall be used to record information on data forms and the LRBs. Pencil drafts may be used provided that record originals are prepared by photocopying or through transcription onto another form. If the penciled 
documents are transcribed onto another form, the original pencil document shall also be kept as a record.

\subsection{Corrections to Documentation}

If an error is made on any laboratory documentation, an individual may correct the error by drawing a line through the error and entering the correct information. The error shall not be obliterated. All non-editorial corrections shall be initialed and dated.

\subsection{CALIBRATION PROCEDURES AND FREQUENCY}

A11 measurement and test equipment (M\&TE) must be controlled in accordance with PNL-MA-70 Administrative Procedure PAP-70-1201, Calibration Control System.

\subsection{Thermometer Calibration}

A digital thermometer calibration check must be performed daily before using the thermometer by comparison to a certified mercury thermometer as. specified in MSL-3, Calibration and Use of Thermometers. The calibration must be documented on a Thermometer Calibration Record. In order to confirm that the thermometer(s) are still in calibration, an "as-found" calibration must be performed after completion of an activity.

\subsection{DATA REDUCTION, VALIDATION, AND REPORTING}

\subsection{Data Management Procedures}

The following shall be performed for verification of data input into spreadsheets and/or data bases:

The staff member entering data shall assure correct entry into the software by comparing data with the hard copy of the data listing. If errors are discovered, the errors shall be corrected and a new data listing generated. When data are correctly entered, the staff member entering the data shall sign and date the correct data listing and submit it as a project record.

Data entered on this software shall be backed up on a daily basis or as needed.

Manipulation of data within the spreadsheets shall be verified with hand calculations. These calculations shall be documented directly on the printouts or shall be traceable to the printouts and shall be checked as a primary verification of accuracy and completeness. Checking shall be performed by qualified persons who did not participate in performing the calculations. Checking shall be documented, preferably by signature and date on the printout. Separate documentation is acceptable, provided that traceable records are maintained.

\subsection{Process for Handling Suspect or Unacceptable Data}

When the initial data review identifies suspect data, that data must be investigated to establish whether it reflects true conditions or an error. The investigation shall be documented. If the data value is determined to be 
in error, the source of the error must be investigated, the correct value established if possible, and the erroneous value replaced with the correct value. If the investigation concludes that the data are suspect (possibly in error) but a correct value cannot be determined, the data must be flagged to indicate its suspect status.

\subsection{Standard Units}

The standard units used to report data are:

Chemistry parameters

Total Organic Carbon

0 il \& Grease

Petroleum Hydrocarbons

Metals

Butyltins

Semivolatile Organic Compounds (PAH)

Chlorinated pesticides \& PCBs

Grain size

\begin{tabular}{ccc} 
Sediment & & Water \\
\cline { 1 - 1 }$\%$ & & $\mathrm{mg} / \mathrm{L}$ \\
$\mathrm{mg} / \mathrm{kg}$ & & $\mathrm{mg} / \mathrm{L}$ \\
$\mathrm{mg} / \mathrm{kg}$ & & $\mathrm{mg} / \mathrm{L}$ \\
$\mathrm{mg} / \mathrm{kg}$ & & $\mu \mathrm{g} / \mathrm{L}$ \\
$\mu \mathrm{g} / \mathrm{kg}$ & & $\mathrm{ng} / \mathrm{L}$ \\
$\mu \mathrm{g} / \mathrm{kg}$ & & $\mu \mathrm{g} / \mathrm{L}$ \\
$\mu \mathrm{g} / \mathrm{kg}$ & & $\mu \mathrm{g} / \mathrm{L}$ \\
$\%$ & & $\mathrm{~N} / \mathrm{A}$
\end{tabular}

\subsection{Reports}

In addition to the requirements contained in the Scope of Services for reports issued to the USACE, the following shall apply:

The final report to the client shall contain citations to the methodology used (i.e., EPA, ASTM) on the technical activities for this project.

The report shall undergo internal independent technical review. The Program Manager shall select technical reviewers who will be able to assure that the report is technically adequate, complete, and correct. Selection shall be based on:

- technologies and disciplines represented in the report.

- qualifications of the reviewer(s). Those selected shall have proven competence in the subject matter of the report, and shall have been given an adequate understanding of the requirements for and objectives of the technical report.

- reviewer independence. Those selected shall be independent of the original work performed. 


\subsection{INTERNAL QUALITY CONTROL (QC) CHECKS}

As an absolute minimum, the QC checks presented in Table 9.1 shall be followed when performing the chemical analysis of the sediment and water samples.

Table 9.1. Summary of Qual ity Control Checks

Data Characteristic Evaluated

Laboratory Contamination

Laboratory Accuracy

Laboratory Accuracy

Precision (lab variability)
Sample Type

Lab Blank

Standard Reference

Material

Spikes

Lab Duplicates
Frequency

1 per batch

1 per batch or

1 per 20 samples

1 per 10 samples

1 per 20 samples

For laboratory accuracy, the concentration of the spike shall be large enough to increase the analyte's concentration by at least 50 percent, but not more than 200 percent above the original sample concentration. If the sample concentration is less than three times that of the analyte, then a spike of two to three times that detection limit is appropriate.

Printouts from all $A A$ and $G C$ analyses shall be kept on file in the event that any concerns arise with the data.

All laboratory analyses shall be completed within the recommended holding time for each analytical method.

All GC analyses require confirmation using a second column which is different from the one used in the initial GC analyses

\subsection{SPECIFIC ROUTINE PROCEDURES USED TO ASSESS DATA PRECISION, ACCURACY, AND COMPLETENESS}

Because of the nature of environmental measurements, it is difficult or impossible to know the "true" value of the measured parameter. The accuracy of the measured value must instead be inferred through the use of QC samples of known composition. This project uses this method to verify that the data quality objectives (DQOs) established in Table 4.1 have been met.

Since this project is not a monitoring project, routine procedures to monitor data precision, accuracy, and completeness are not required. Precision, accuracy, and completeness will be calculated following equations presented in section 4 and the results will be reported in quality control tables in the final report. These results will be compared against the DQOs established in Table 4.1 and this comparison will also be reported in the final report.

\subsection{RECORDS}

Records shall be indexed and subsequently maintained in accordance with PNLMA-68, Records Management. All project records shall be transferred to the DOE records holding area within 90 days after project completion and/or after 
client approval of the final report. The retention period for storage at the DOE records holding area shall be specified on the Records

Inventory/Disposition Schedule (RIDS). Records will not be turned over to the client unless specifically requested. The project Quality Engineer does not have to approve the RIDS.

\subsection{PROCUREMENT CONTROL}

\subsection{Purchase Requisitions and Subcontractors}

Procurements of items and subcontracted services are governed by PNL-MA-70 Administrative Procedure PAP-70-401, Preparation, Review, and Approval of Purchase Requisitions.

All subcontractors used by PNL shall follow the applicable requirements as delineated in this QA Plan. For subcontractors that will be performing chemical analysis, a preaward evaluation of the capabilities of that supplier shall be made by either a representative of the Quality Control group or by the Quality Engineer.

\subsection{STAFF TRAINING}

Staff performing activities affecting quality shall be trained for applicable technical disciplines that he \she is working on.

Current resume's of key project staff shall be maintained in the project files. As a minimum, the resume shall contain the following:

- education completed (e.g., degree and major)

- work experience (employer and major responsibilities)

- licenses and certifications

- related training and qualifications

- applicable dates associated with the above information 


\section{APPENDIX B}

CHAIN-OF-CUSTODY FORMS 


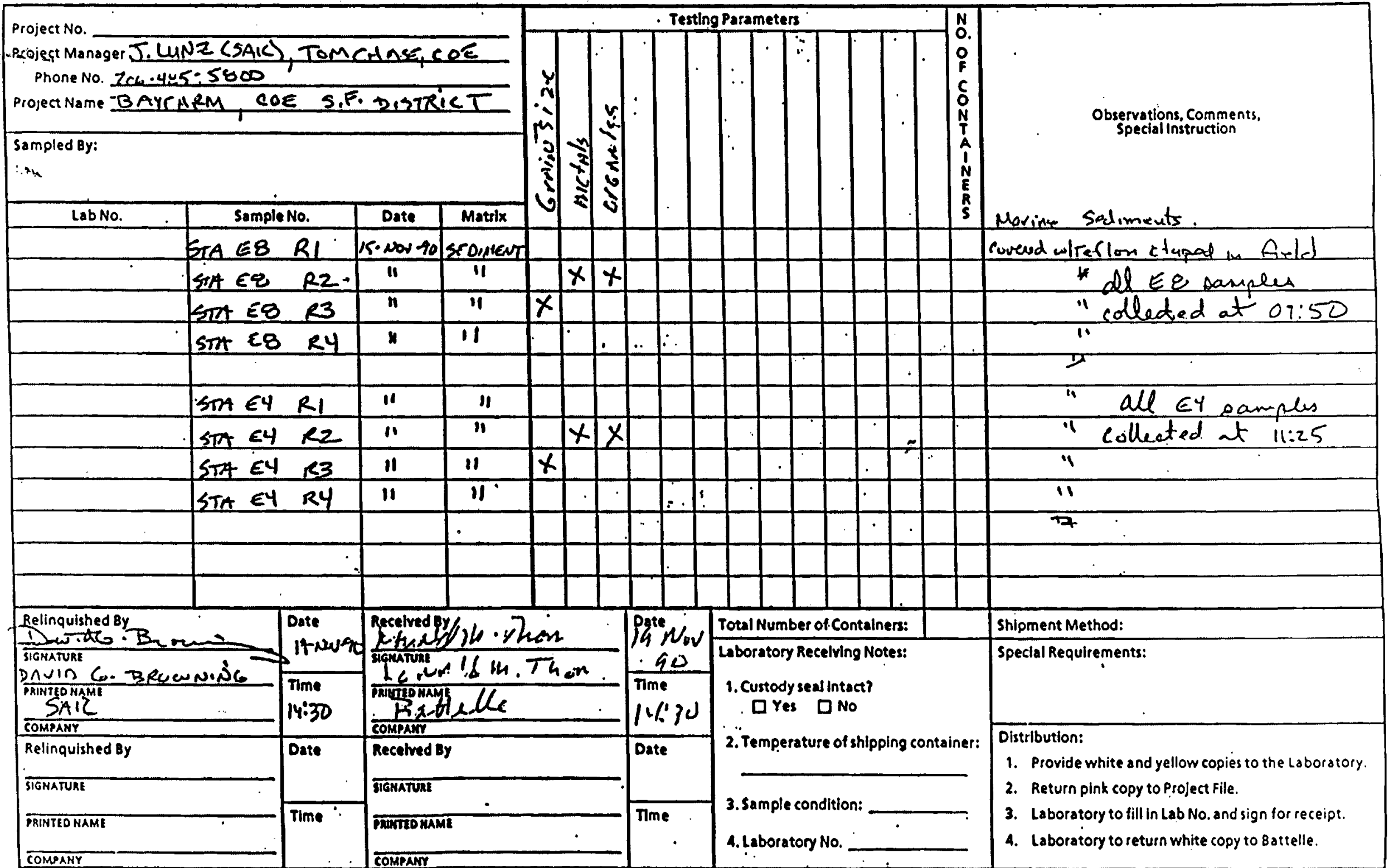




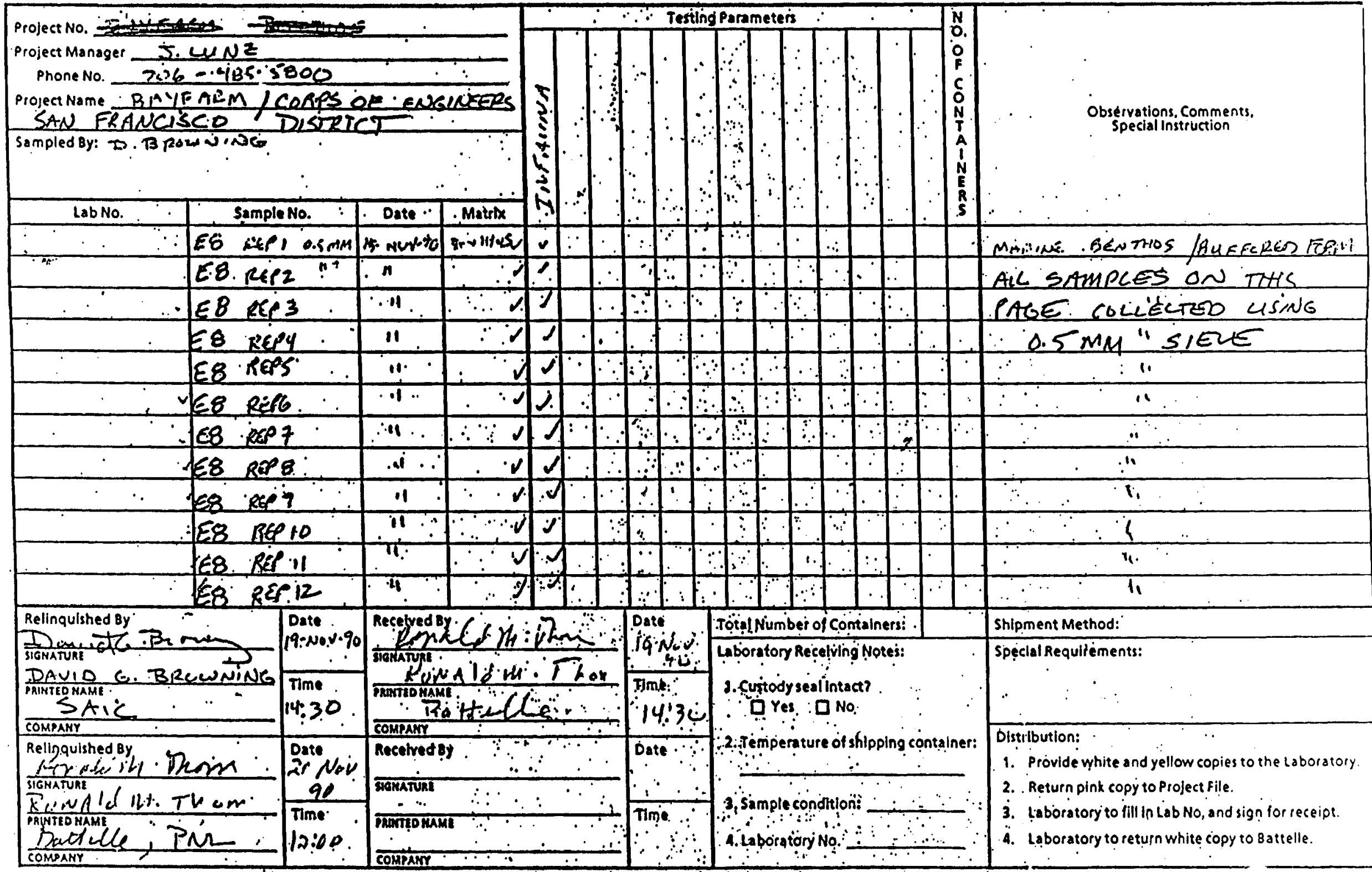




\begin{tabular}{|c|c|c|c|}
\hline Lab No. & Sample NO. & Date. & $\because$ Matrix \\
\hline & ES REP 13 ditm & S.Abuto & BENTHBS \\
\hline & EQ Repit os & 11 & $n<$ \\
\hline & EB RCP is of & II & $n$ \\
\hline & E REPI OS & $\|$ & 11 \\
\hline & E4 REPZ. & $\|$ & $n$ \\
\hline & $E 4$ LCA & 11 & $n$ \\
\hline . & E4 Rep4 & 11 & $\dot{n}$ \\
\hline & E4 REPS & $\pi$ & $n$ \\
\hline - & EA REPG & $n$ & $\because$ \\
\hline & E4 REP7 & 11 & 11 \\
\hline & E4 REB 8 & $\pi$ & $\pi$ \\
\hline & E4 REP 9 & !) & 11. \\
\hline
\end{tabular}

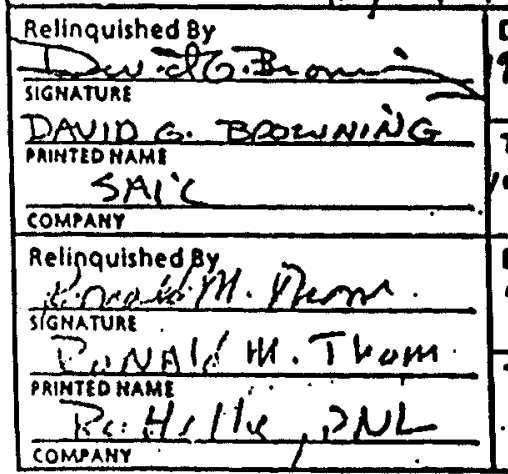

\section{Testing Parameters}
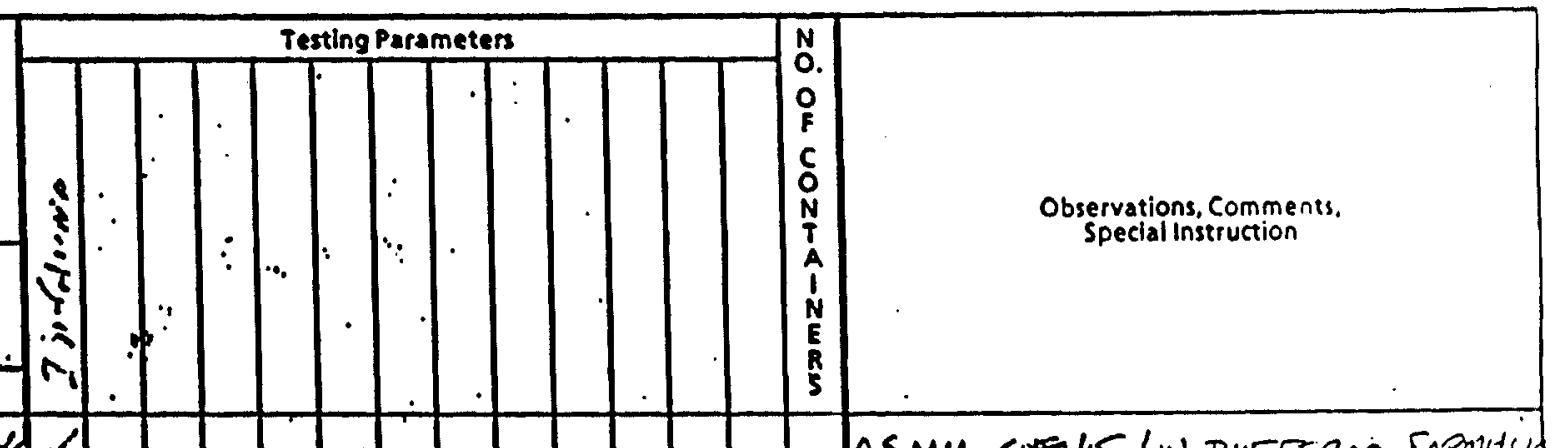

O.SMM SIEVE/W BUFFERED TORnituid

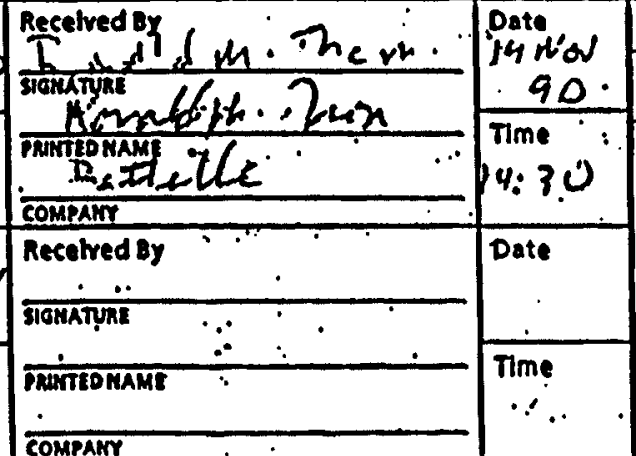

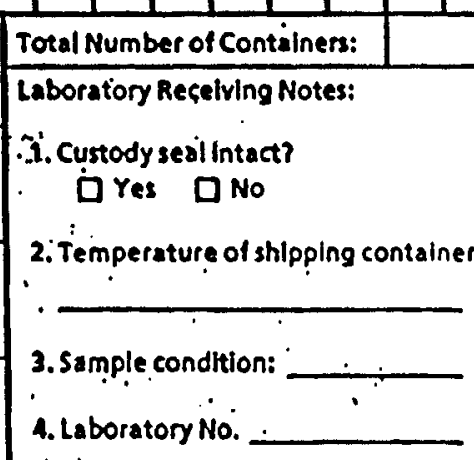

Total Number of Containers:

9 - $k$ ku- 90

Time $4: 30$ Date Nov

90
$12: 00$

companr
Distilbution:

1. Provide white and yellow copies to the Laboratory

2. Return pink copy to Project File.

3. Laboratory to fill in Lab No. and sign for receipt.

4. Laboratory to return white copy to battelle. 


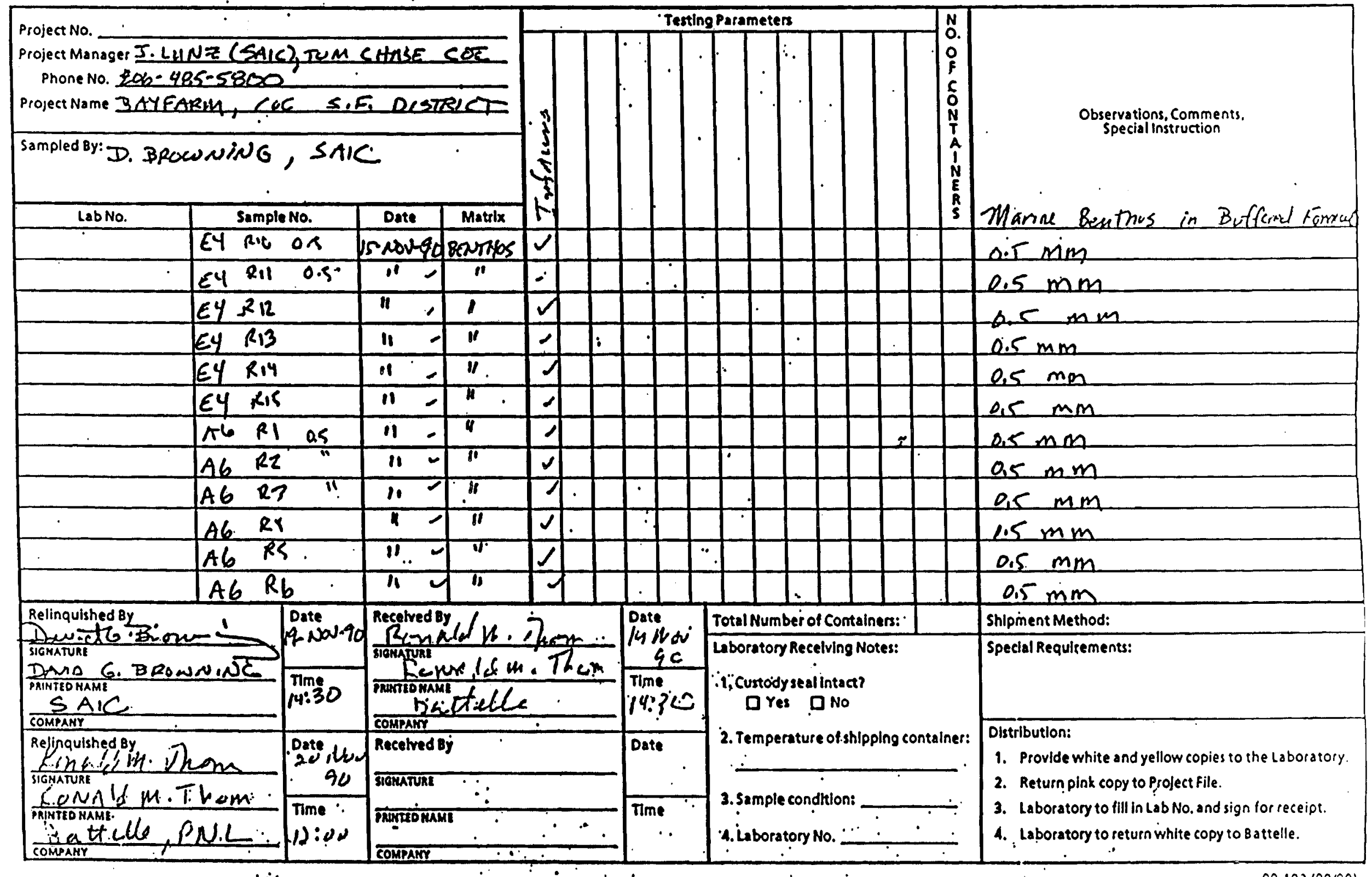


$\because \because$ Testing Pajameters

Phone No. $206-485-5800$

Project Name BAYEAEM /CQE SE PISTRLT:

SampledBy:DAUID BRDWNint SAlC

\begin{tabular}{|c|c|c|c|}
\hline Lab No. & Sample No. & Dite & Matrix \\
\hline & iis $R 7$ as & $15-A b r-90$ & 3 ENTTfOS \\
\hline & $A 628$. & $\cdot 0$ & $" 6$ \\
\hline & Atb R9 il & 1 & $n \cdot v$ \\
\hline & $A_{16} R_{10} " 1$ & 1 & 11 \\
\hline & Ald RII " & 11 & $11 \quad \dot{v}$ \\
\hline & $A 6$ R12 1 & 11. & 11.2 \\
\hline & $\begin{array}{lll}A K_{2} & R_{13} & \prime\end{array}$ & "1 & If \\
\hline & Ab R4. & '1: & $\boldsymbol{H}$ \\
\hline & 46 RK " & 11 & of \\
\hline & GIO RI $0: 5$ & & 11 \\
\hline & $610 \mathrm{e2}$ & & Y.: \\
\hline & G10.23 & & .11 \\
\hline
\end{tabular}

\begin{tabular}{|c|c|}
\hline 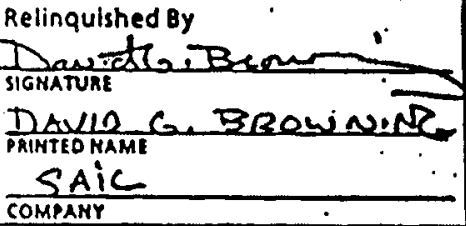 & Date \\
\hline 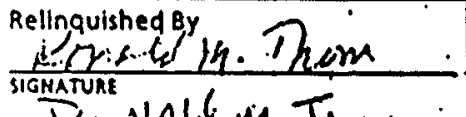 & $\begin{array}{l}\text { Datejo } \\
\text { nis } \\
\text { and }\end{array}$ \\
\hline PRITTEOAME & $\begin{array}{l}\text { Time: } \\
12: 00\end{array}$ \\
\hline
\end{tabular}

\section{Recefved ay. tival.}

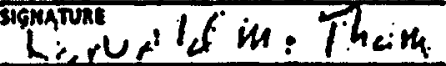

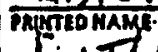
int 1

Recelved ay Recetreg ay

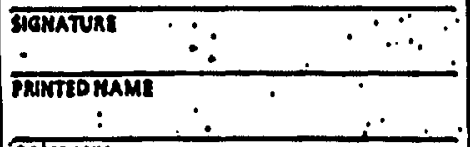

colpasr
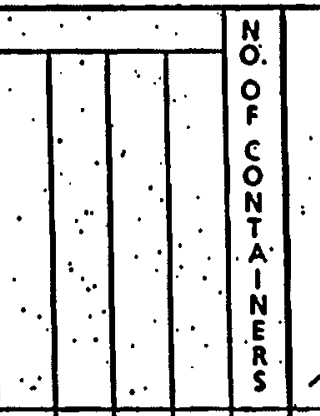

Marine Basthesio Bufferd Enmuliss $0.5 \mathrm{~mm}$ $0.5: \mathrm{mm}$ $0.5 \mathrm{~mm}$ 0.5 man $0.5 \mathrm{~mm}$ $0.5 \mathrm{~mm}$ $0.5 \mathrm{~mm}$ $0.5 \mathrm{~mm}$ $0.5 \mathrm{~mm}$ $0.5 \mathrm{~mm}$ $0.5 \mathrm{~mm}$

0,5 min Shipment Method:

Speclal Requitiements:

$\therefore$ प' Yes no

2. Temperature of shippling container;

Olistribution:

i. Provide witte and yellow copies to the Laboratory.

3.sumplécondtion:

2. Return pink copy to Project file.

-3. Laboratory to fill in Lab No. and sign for receipt.

A. Laboratory toreturn white copy to Battelle. 


\section{SAMPLE CUSTODY RECORD}

$$
\text { Page } 5 \text { of } 8^{P B} \text { Date } a_{:}
$$

Pacilic Northwest Divisio Marine Sciences Laboralory 139 Wesl Sequim Bay Rord

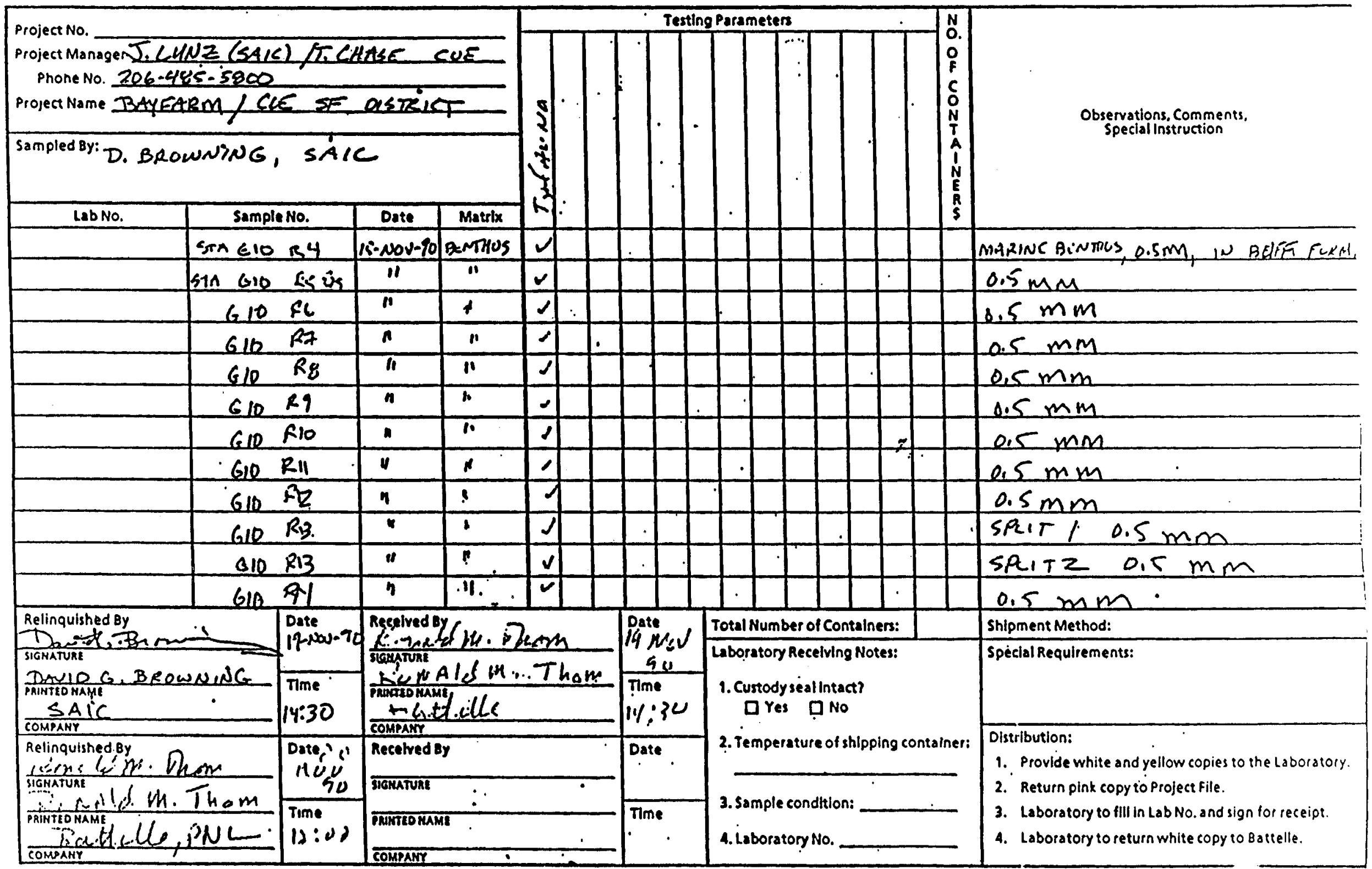




\section{SAMPLE CUSTODY RECORD}

page 6 :

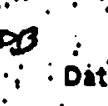

$\therefore$

$\therefore$

Battelle

Pacille Northwest Division Masine selences Liboralory 439 Wesl sequim Bay Road sequim. Washingion 9838?

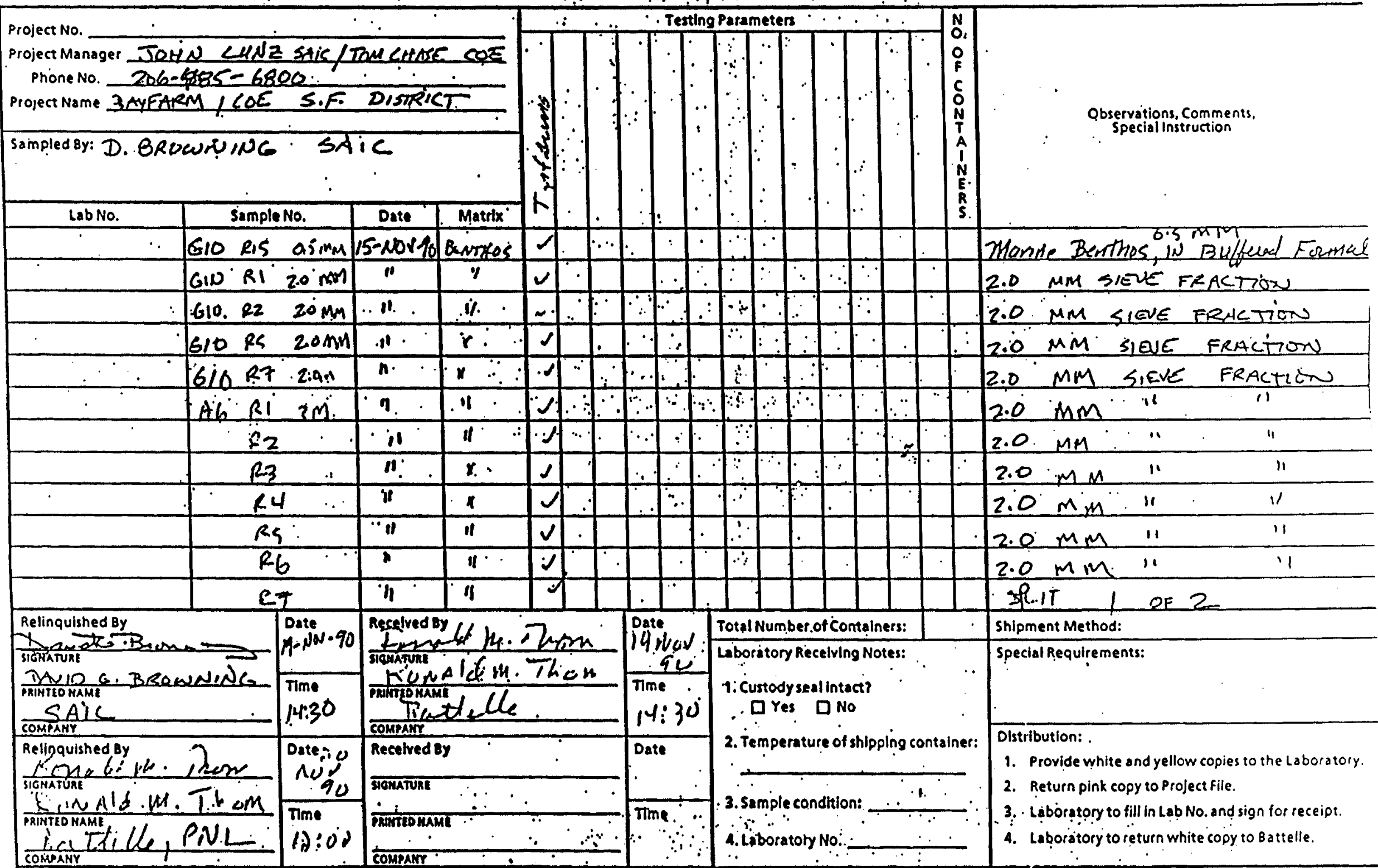


. Testing pirameters

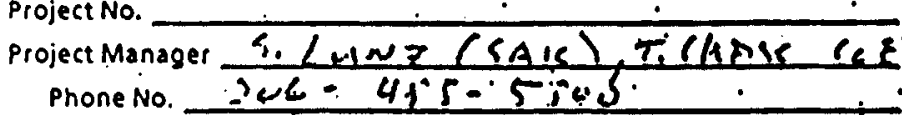

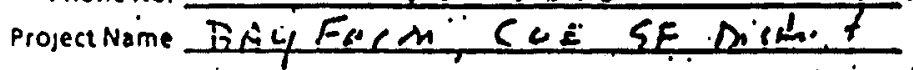

sampled By:

D. Broweriagl stic

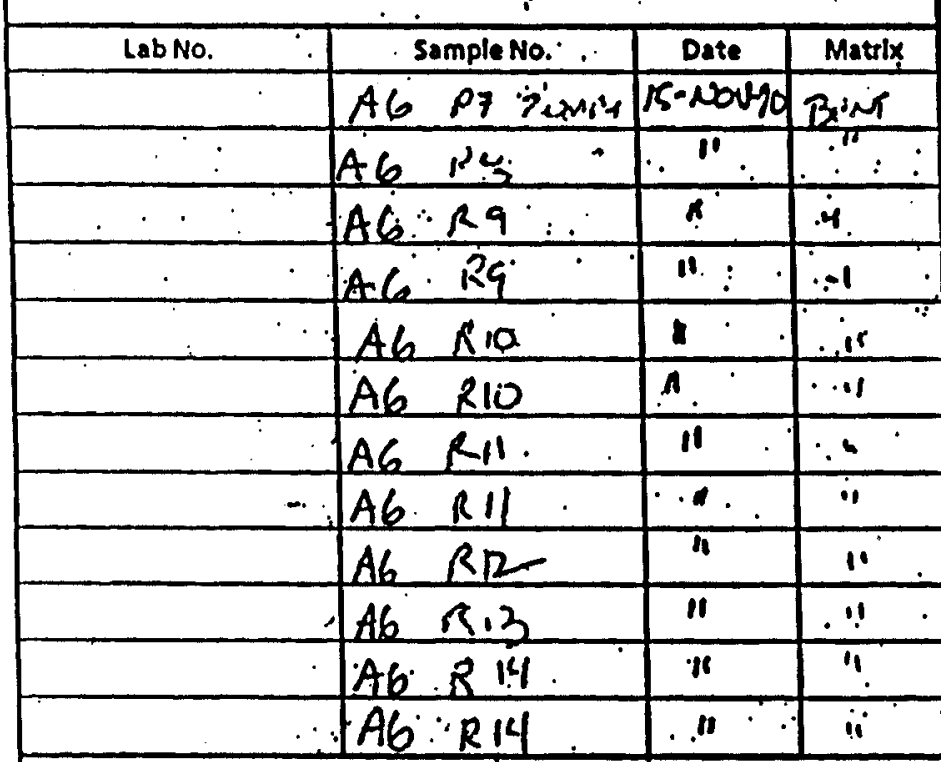

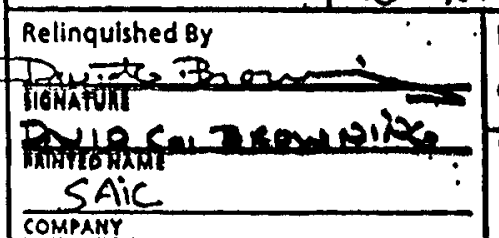

Date $19-N$ N.

Regegred by

M-NW.10

Mr. 30

Relinquished By H. Rom sigparune 1. in. T.6 oin $\mathrm{Mr} 3 \mathrm{O}$

IIstavi
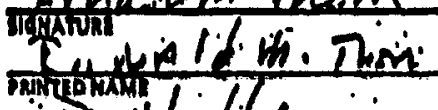

Datel a:

- Routticle

Recotved oy
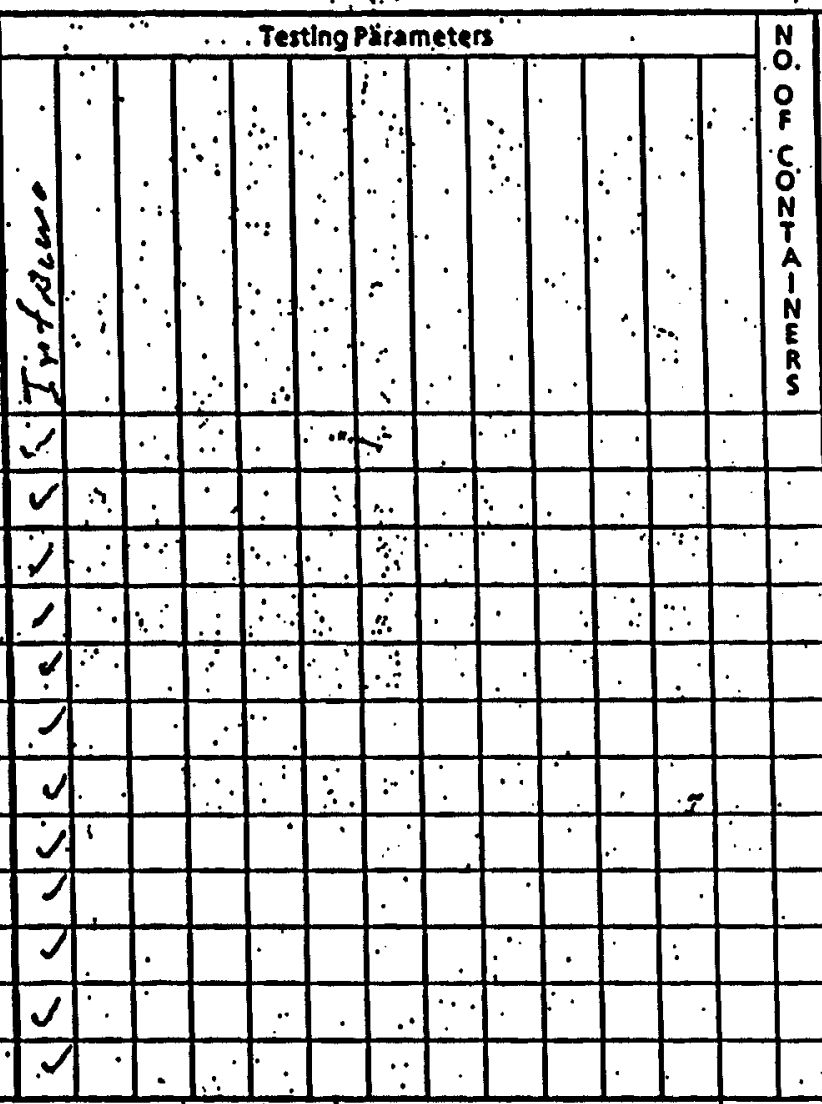

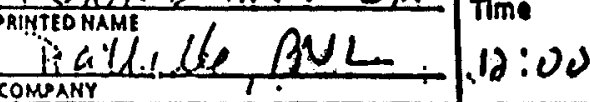

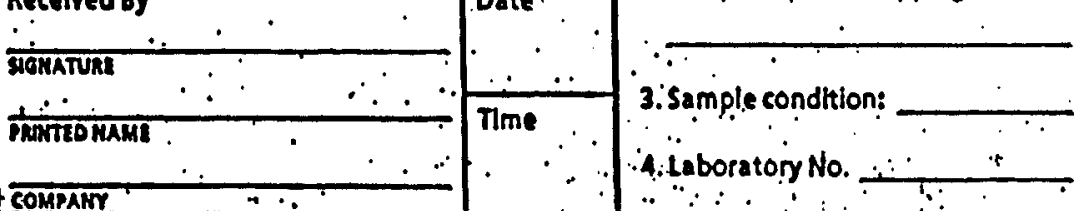

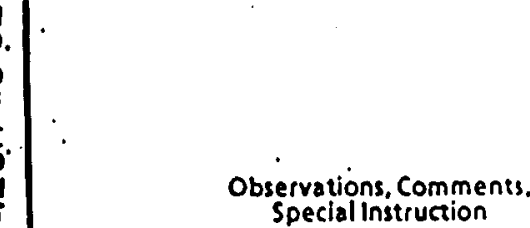

Special Instruction

Mouine BenThos, in Buffuad Foumalin SBLIT 2:20 $\mathrm{mm}$

AfExza $7.0 \mathrm{Mm}$

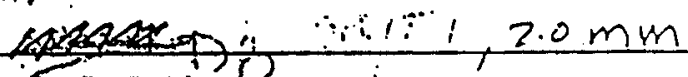

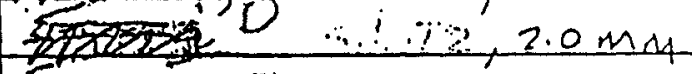

Bftis ….......

$591 \mathrm{~T} 12.0 \mathrm{~mm}$

$\operatorname{spon} 2$

$2.0 \mathrm{~mm}$

$2.0 \mathrm{mnd}$

$2.0 \mathrm{~mm}$

iplit. $1,2.0 \mathrm{~mm}$

Date: Total Number of Contalners:

Laboratory Recelylng Notes:

Shlpment Method:

i. Custodyseal intact?

D Yes $\square$ No.

2. Temperature of shipping container:

Distribution:

1. Provide white and yellow copies to the Laboratory.

2. Return pink copy to project file.

3. Laboratory to fill in Lab No. and sign for receipt.

4. Laboratory to return white copy to battelle. 
8.

$\$ B$

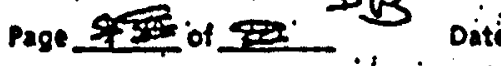

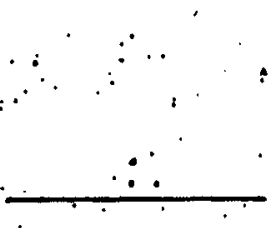

3attelle

Paclific Northwesı Division

Matine Sciences laboralory

139 Wesl sequim Bry Rord

Sequim, Washinglon 98382

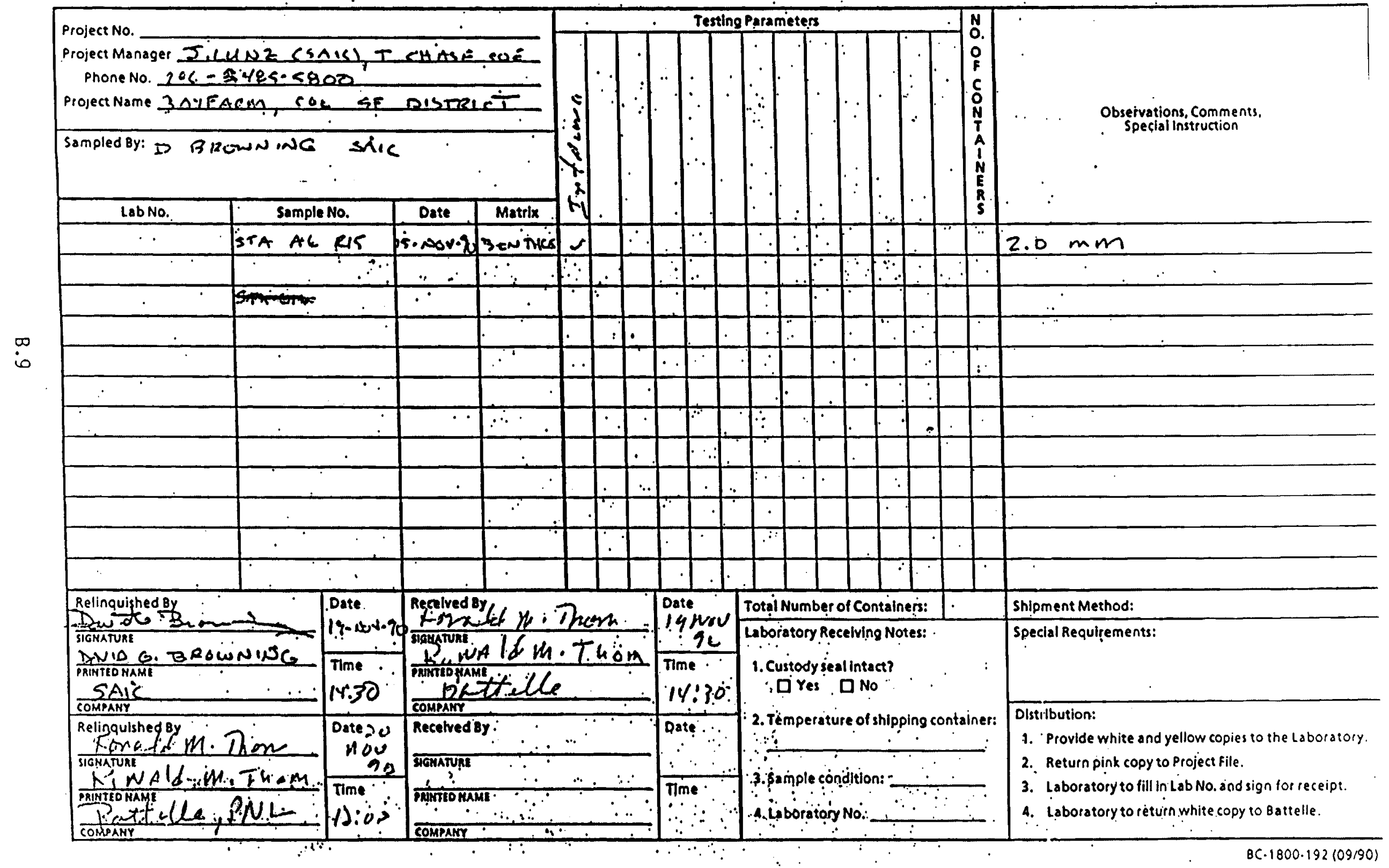




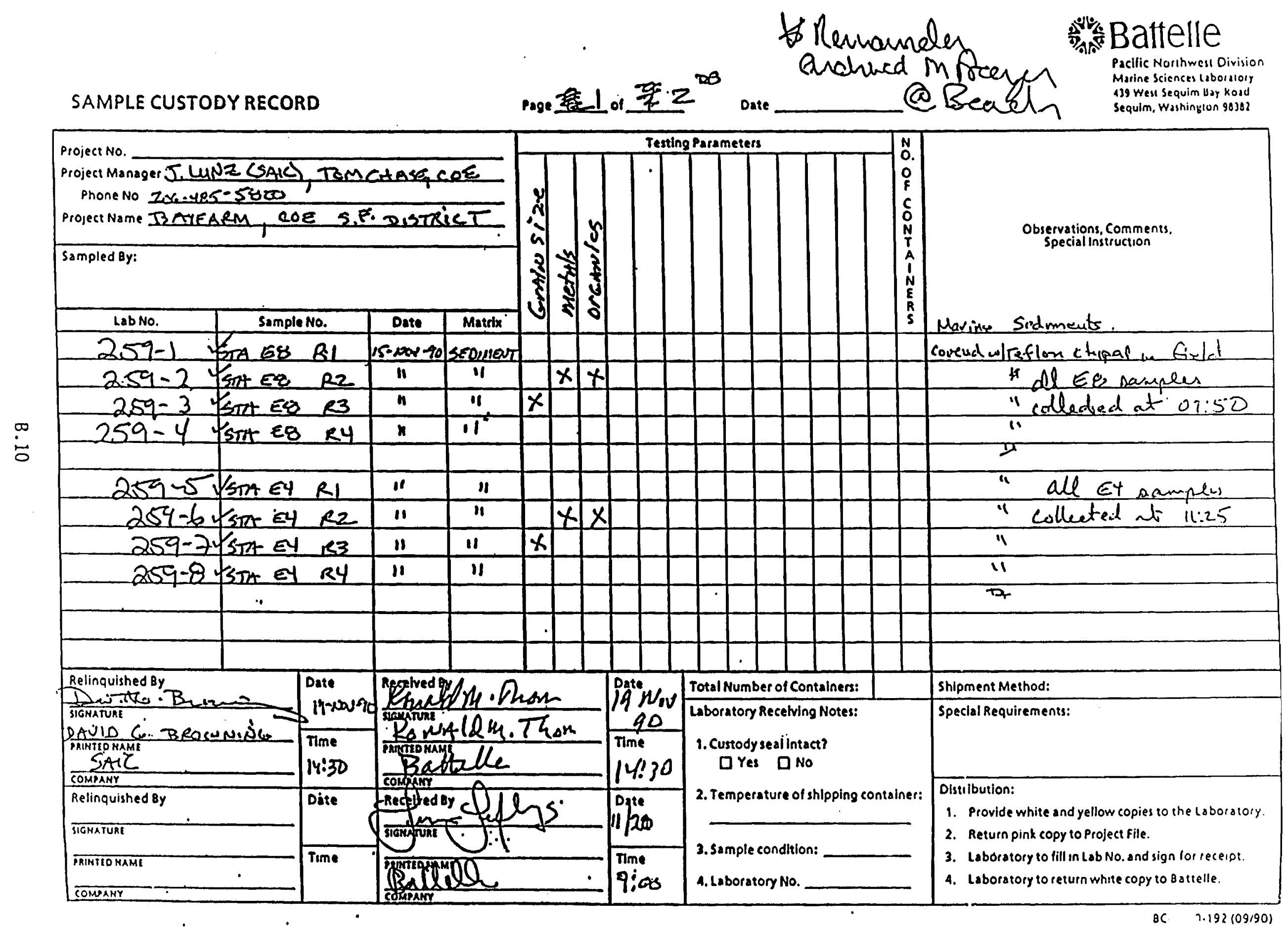




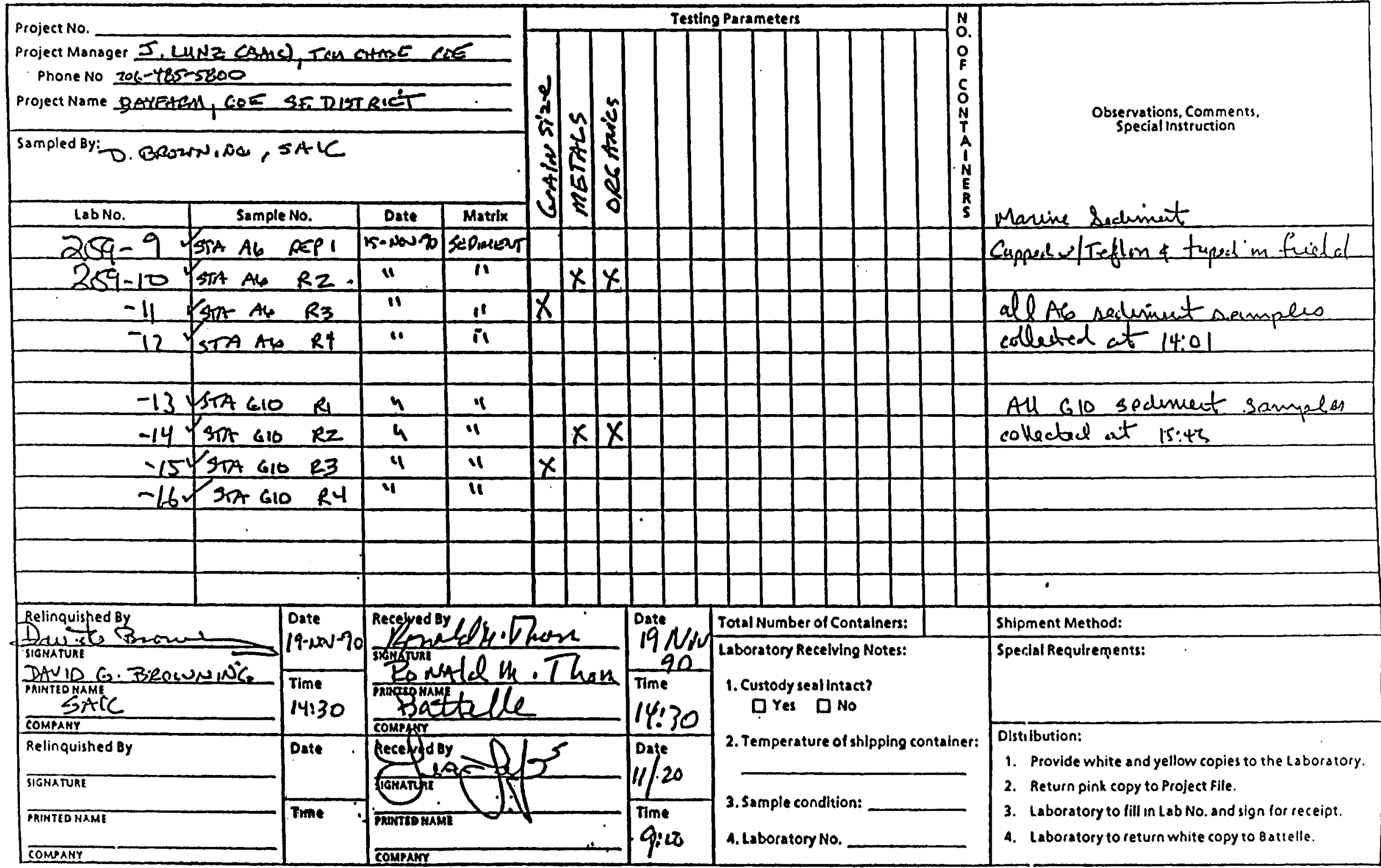




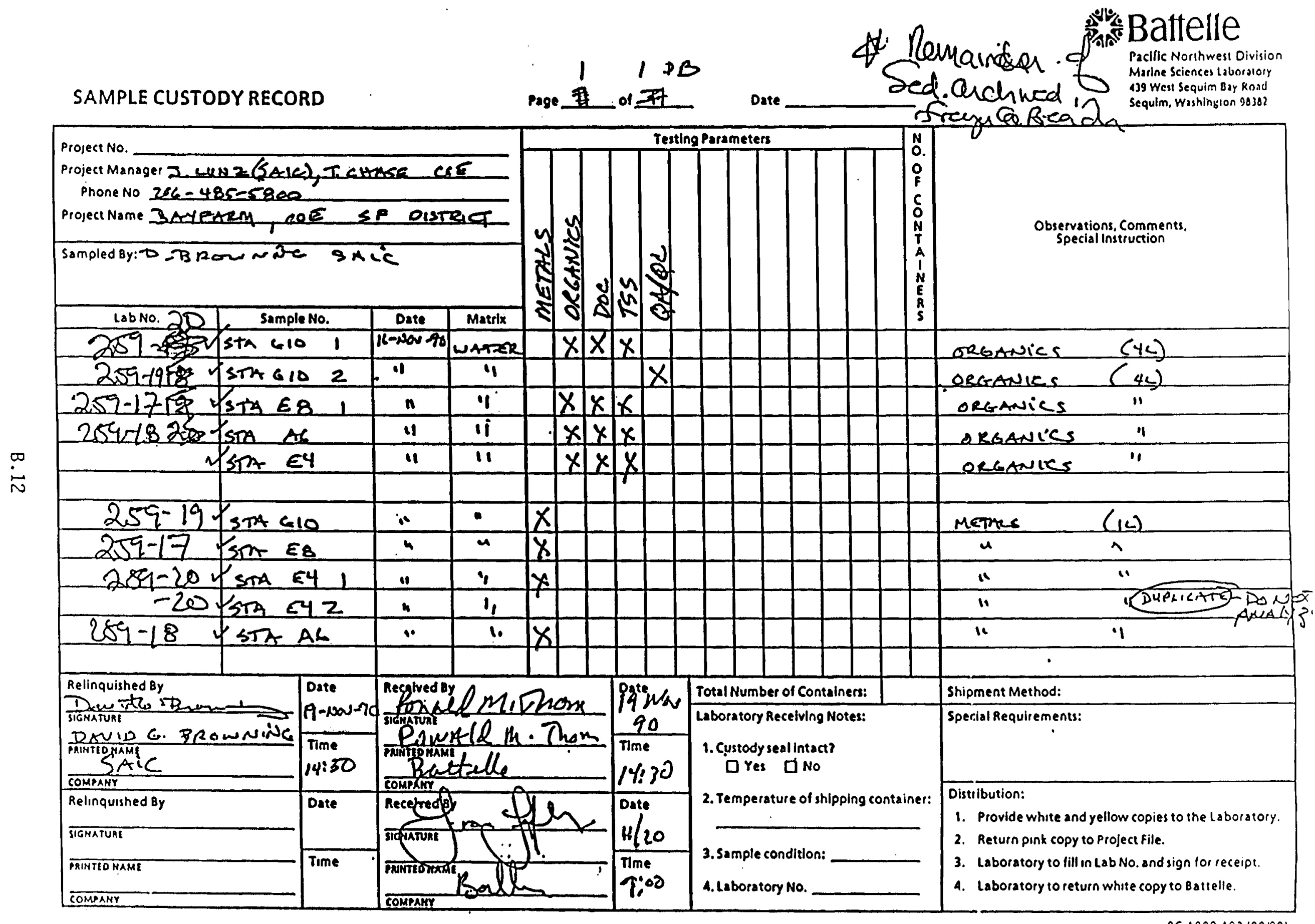




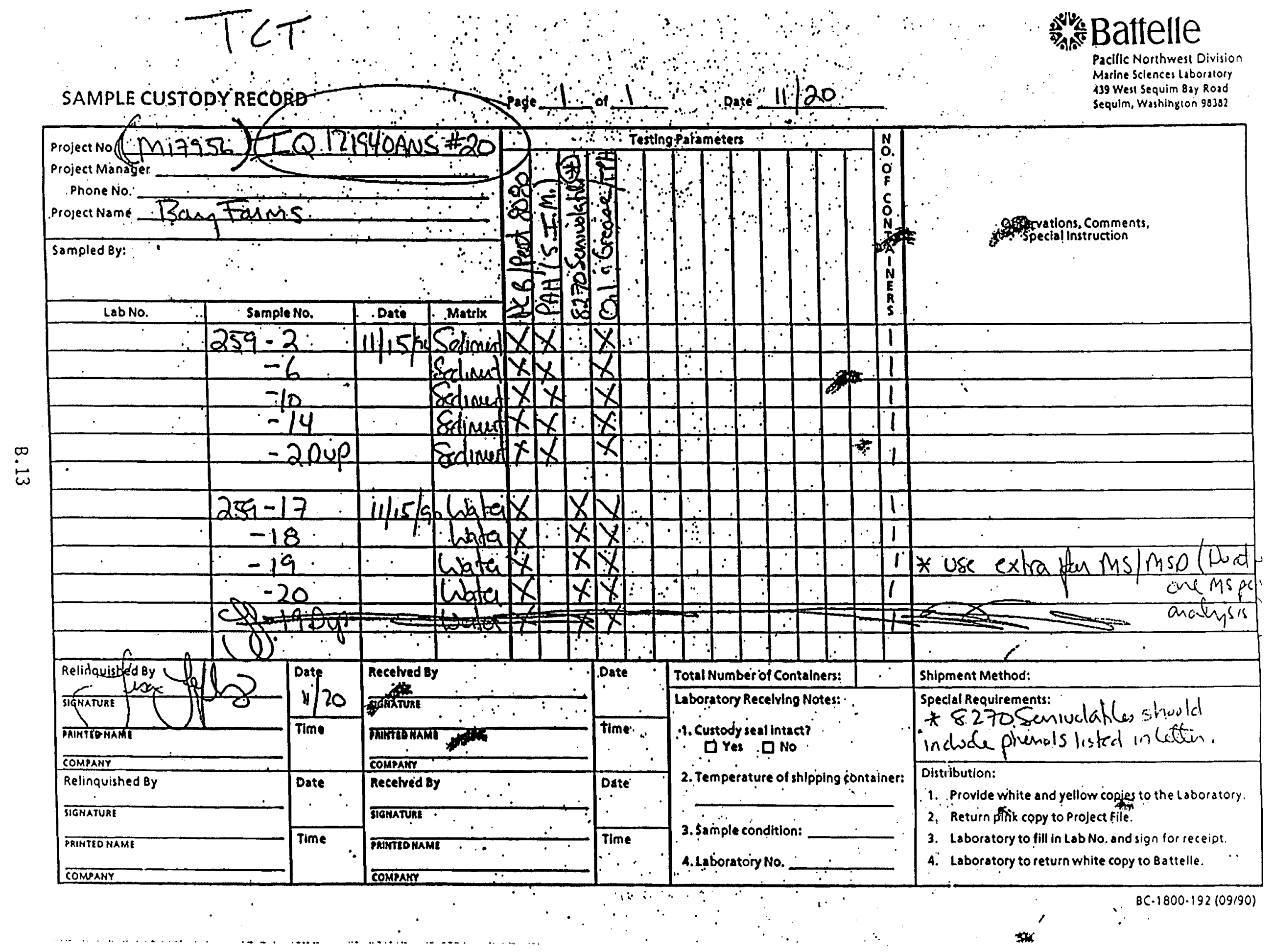


ARI

\section{SAMPLE CUSTODY RECORD}

Project No. M17956

Project Manager

Phone No.

Project Name Bang Trams

sampled By:

$\infty$

$\stackrel{\infty}{\circ}$

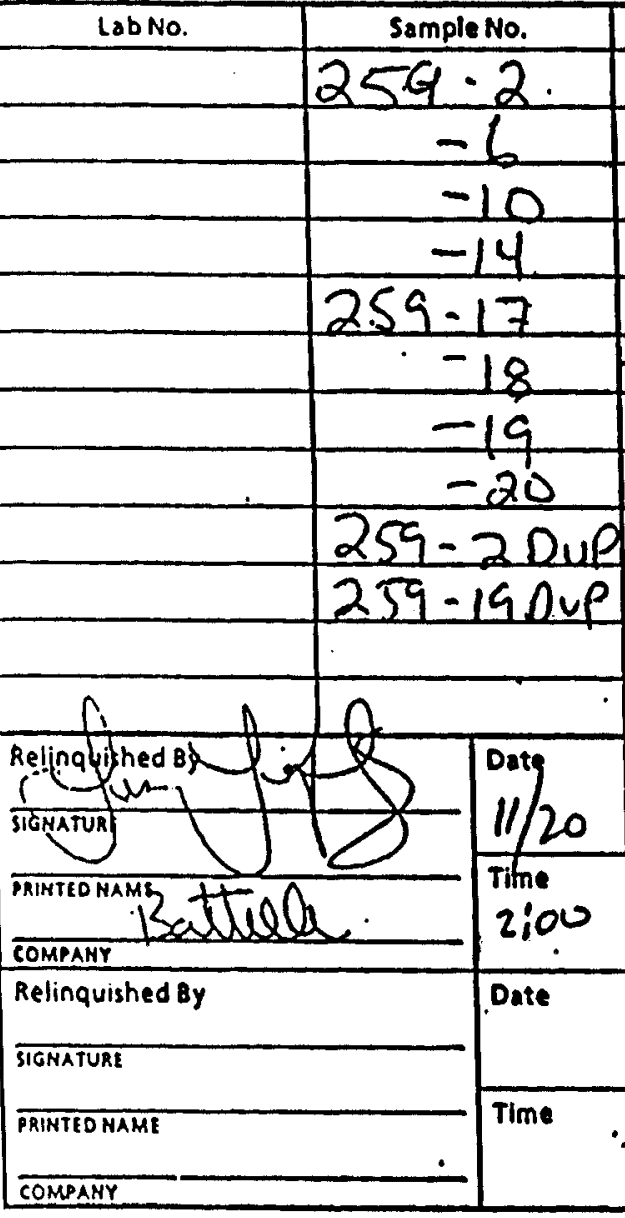

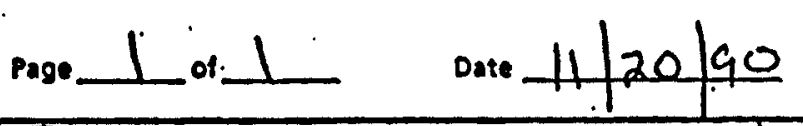

Tenting Paremeters:

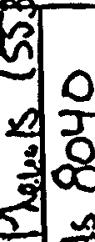

Date Matrix

illis $\operatorname{las}$

redimen $x$

Solion $x \times$

Sedmot $x x$

Setint $x \times$

i.- Wata $x \times x$

Wata $x-x \mid x$

Wiater $x-x$

Waten $x \quad x \mid x$

xdiment $X X$

$v$ water $x$

$x \times$

1 ot

\begin{tabular}{|c|c|c|}
\hline $\begin{array}{c}\text { Recelved } \\
\text { Cy }\end{array}$ & \multirow[t]{2}{*}{ Date } & Total Number of Containers: \\
\hline SIGKATURT & & Laboratory Recelvlng Notes: \\
\hline PRNTED MAMI & \multirow[t]{2}{*}{ Time } & \multirow{3}{*}{$\begin{array}{l}\text { 1. Curtody seal Intact? } \\
\square \text { Yes } \square \text { No } \\
\text { 2. Temperature of shlpping container }\end{array}$} \\
\hline Companr & & \\
\hline Recelyed Dy & \multirow[t]{2}{*}{ Date } & \\
\hline SIGMATUR: & & \multirow[b]{2}{*}{$\begin{array}{l}\text { 3. Sample condition: } \\
\text { 4. Laboratory No. }\end{array}$} \\
\hline PRRTED RAMI & Time & \\
\hline
\end{tabular}

Shipment Method:

Speclal Requirements:

Distilbution:

1. Provide white and yellow copies to the Laboratory.

2. Return pink copy to Project file.

3. Labora tory to fill in Lab NO. and sign for receipt

4. Laboratory to return white copy to Battelle. 
Sou Technolosy

\section{SAMPLE CUSTODY RECORD}

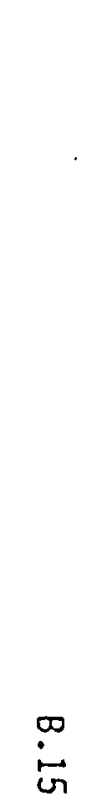

Project No. MI 7556

Project Manager LISA LEFILOUIT

Phone No.

Projecr name Thang Trumis

Sampled 8y:

is troe $1: 1$ of $111 / 20$

B) Battelle

Pacilic Northwest Division

Marine Selencess laboratory

439 West Sequim Bay Road

Sequim, Washingion 98382

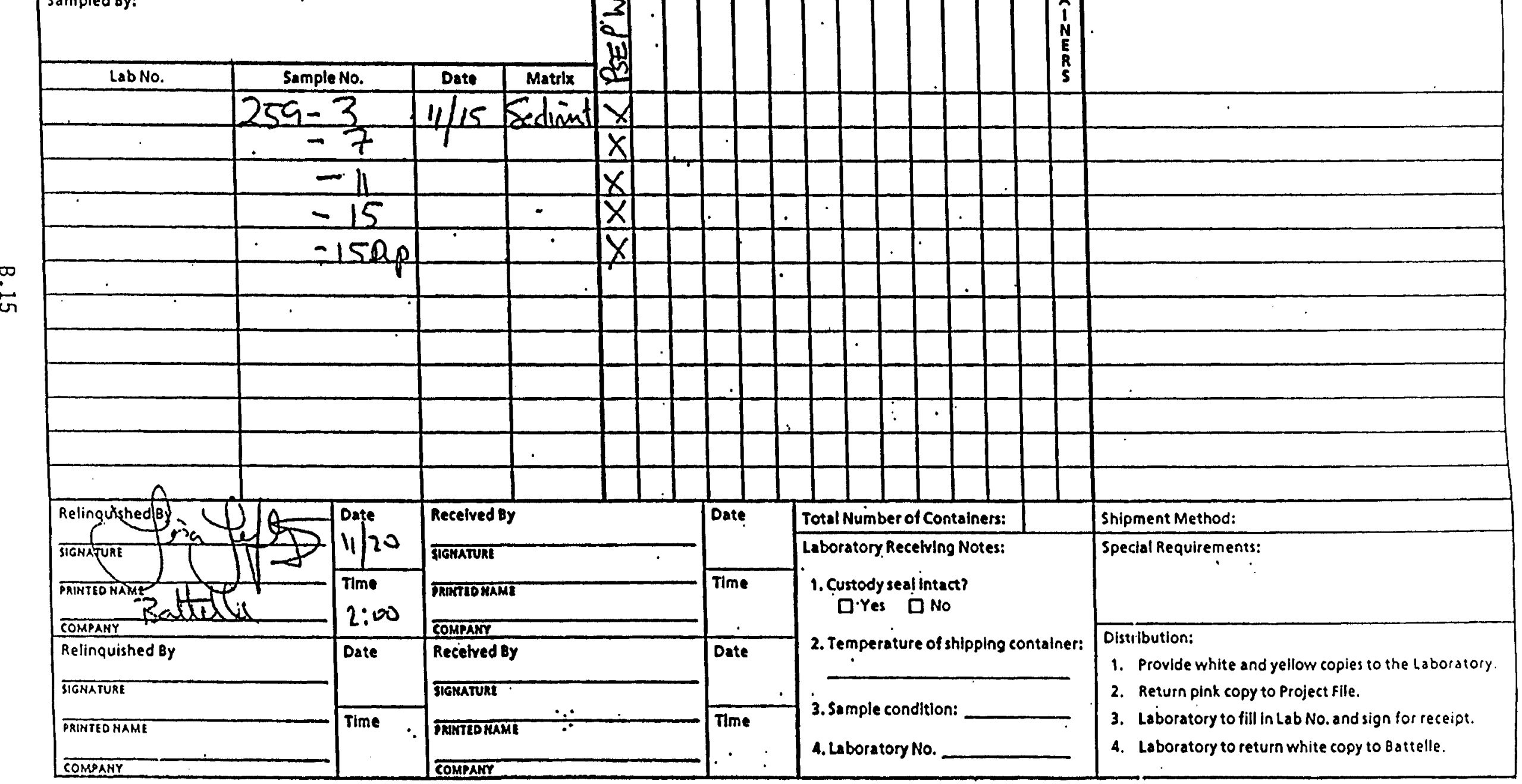

Observations, Comments.

Special Instruction 


$$
x^{2}=
$$




\section{SAMPLE CUSTODY RECORD}

Page $\begin{aligned} & 1 \neq B \\ & \text { of }\end{aligned}$

Pactilic Northwest Division

Marine Sciences Laboratory

439 West Sequim Bay Road

Sequim, Washington 98382

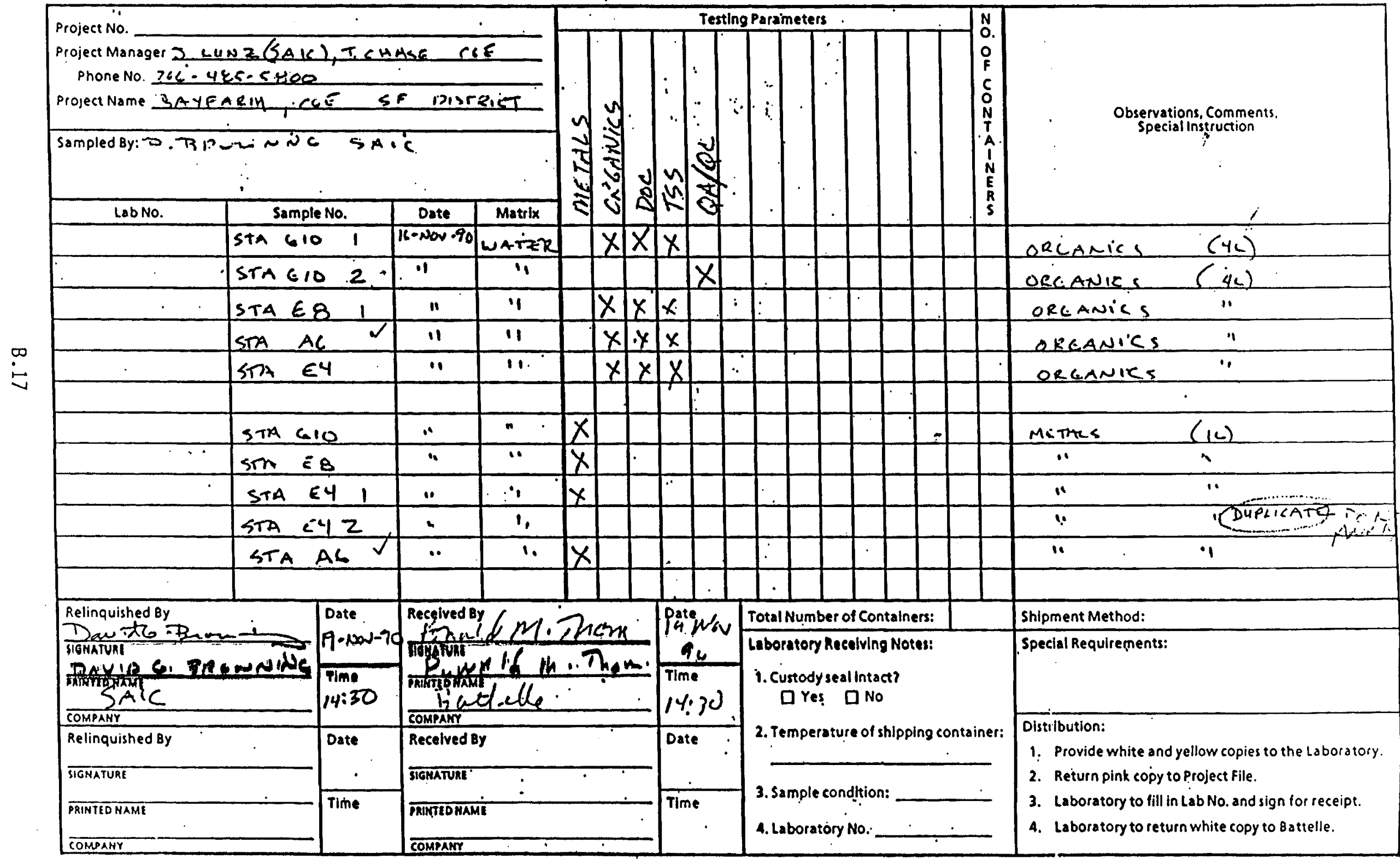




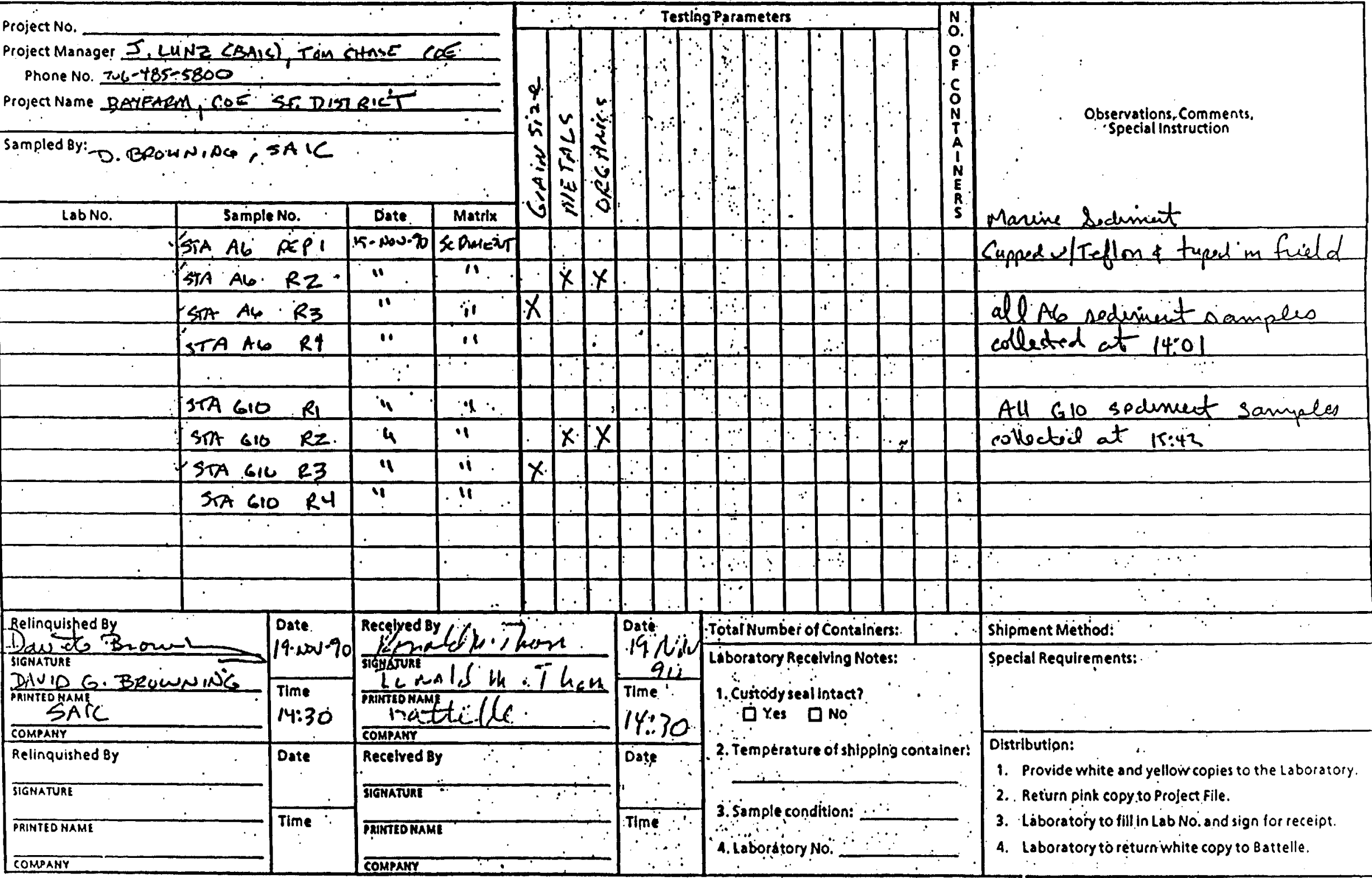




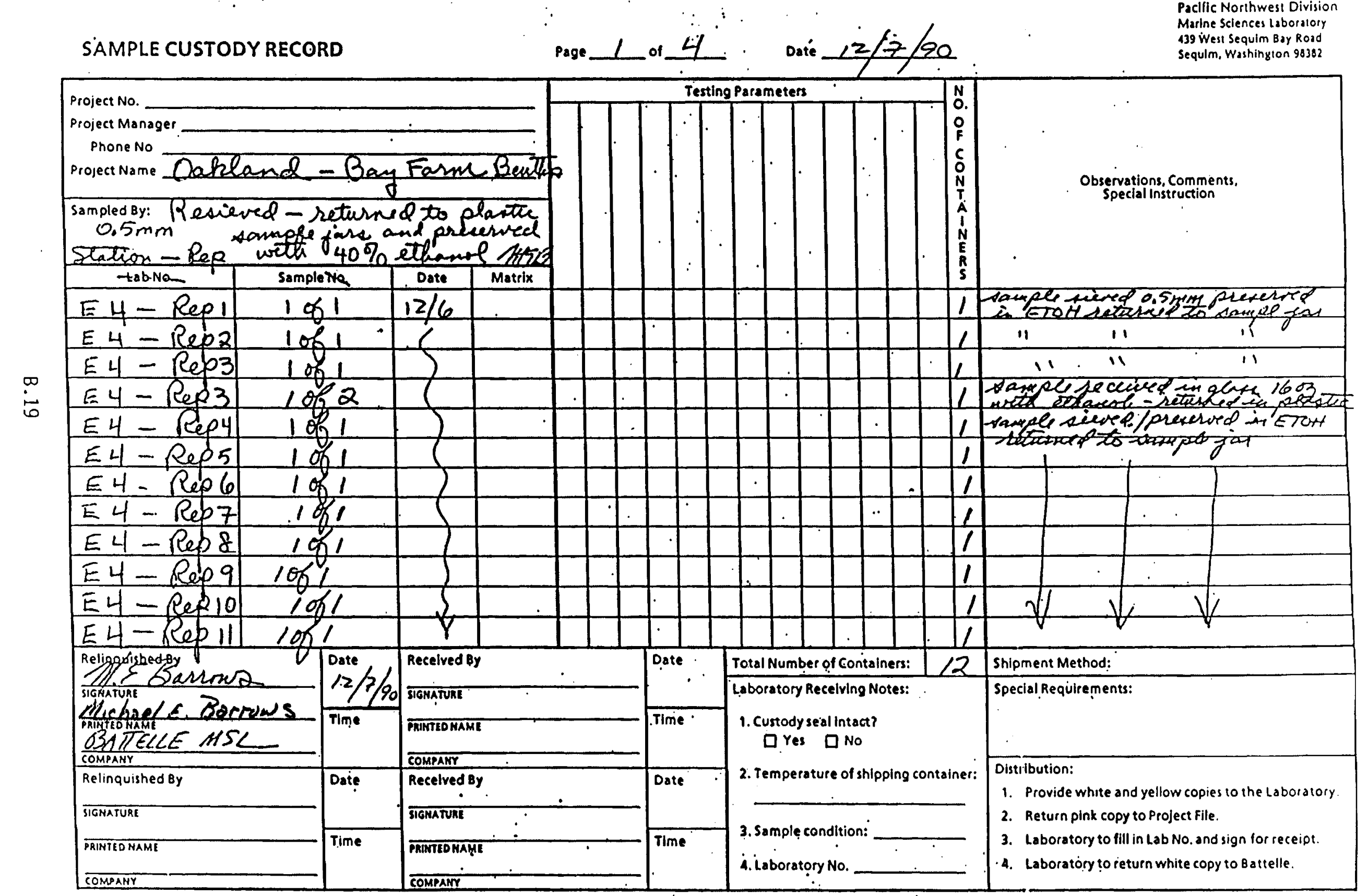



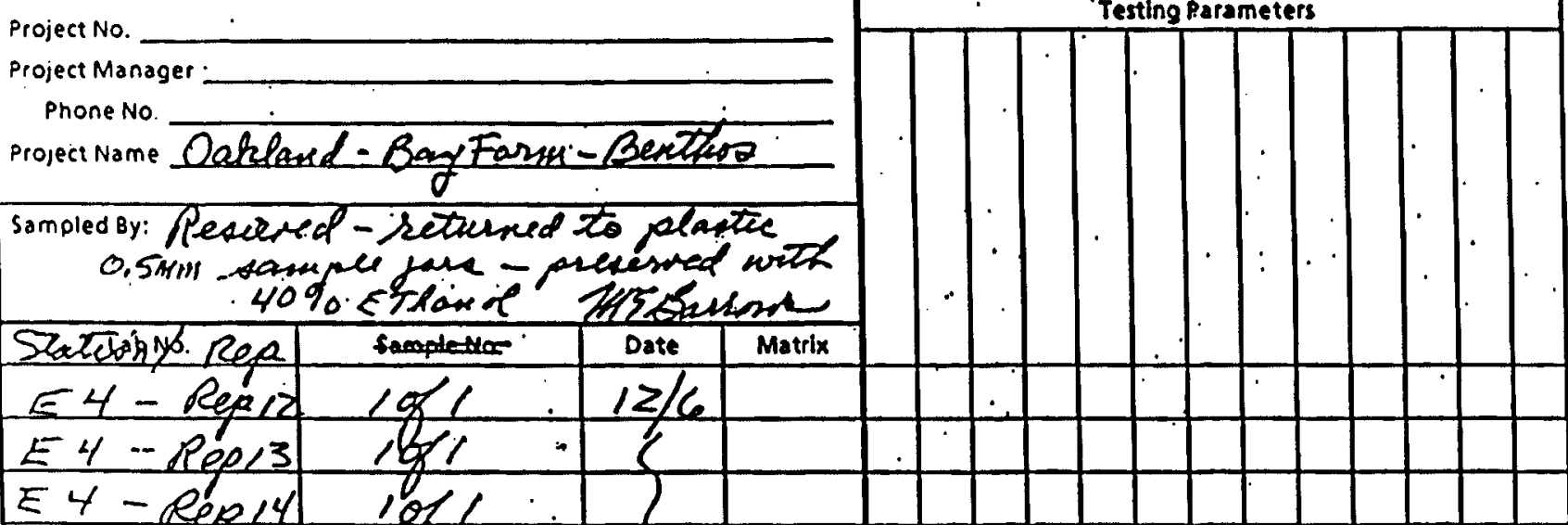

samples fiested $0.5 \mathrm{~mm}$ plexerred in $40 \%$ ETOH = returen do \begin{tabular}{|l|l|}
\hline$E 4-\operatorname{Rep} 14$ & Iof 1. \\
\hline$E 4-\operatorname{sep} 15$ & 101
\end{tabular}

\begin{tabular}{c|c|c} 
& & \\
\hline$A-6-\operatorname{Rep} 1$ & $1001 \rightarrow 2$ & $12 / 7$
\end{tabular}

$A-6-\log 2101 \rightarrow 3312 / 7$

$A-6-\operatorname{cep} 3 \quad$ of $\rightarrow 212 / 6$

$A-6-\operatorname{Reg} 4 \quad 101 \rightarrow 5 / 2 / 6$

$A-6-66051 \% 1 \rightarrow 312 / 6$

$A-6-\operatorname{leg} 6101 \rightarrow 212 / 6$

$A-6-\operatorname{Res} 7$ oseti $\rightarrow 2 \mathrm{r} / 6$

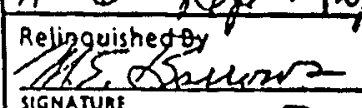

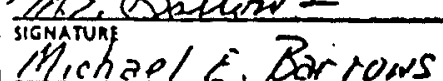
PRINIED NAM! BAITELE AHSL

Relinquished 8y

SIGNATURE

PRINTIO HAME

Date $12 / 7 / 6$

$12 / 7 / 90$ Recelved By SIGMATURE

COMPANY

\section{is 30}

TINTEDNAME

COMPANY Date

Received by

SIGKATUR:

Time

तnirat

comparr

\section{'Testing Rarameters}

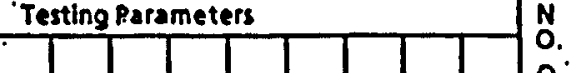

0.

Observations, Comments Special instruction 
$\ldots$

\section{SAMPLE CUSTODY RECORD}

Page 3 of 4 Date $2 / 3 / 90$

3attelle

Pacilic Northwest Division

Marine Sciences labordloiy

439 Wesl Sequim Bay Road

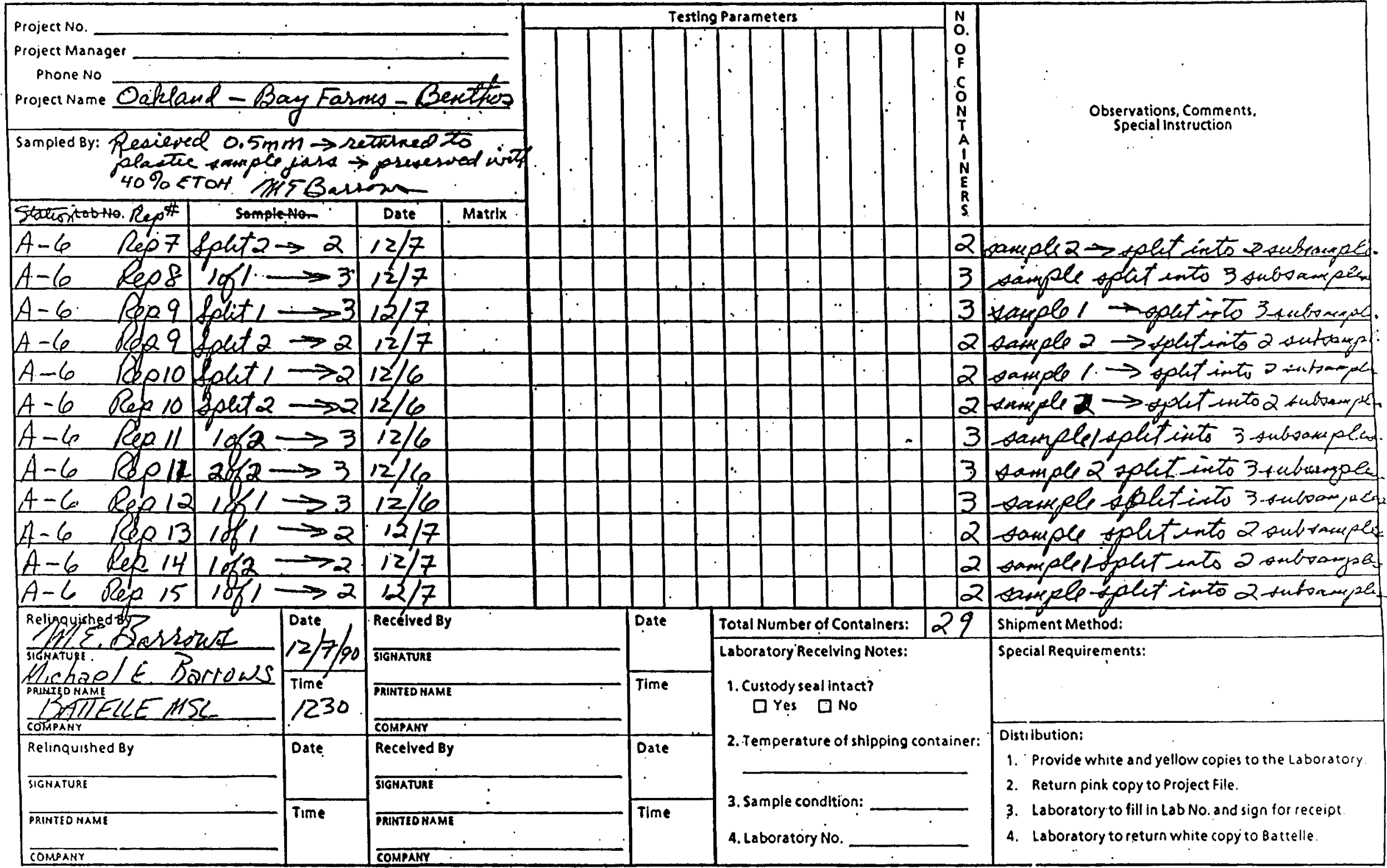




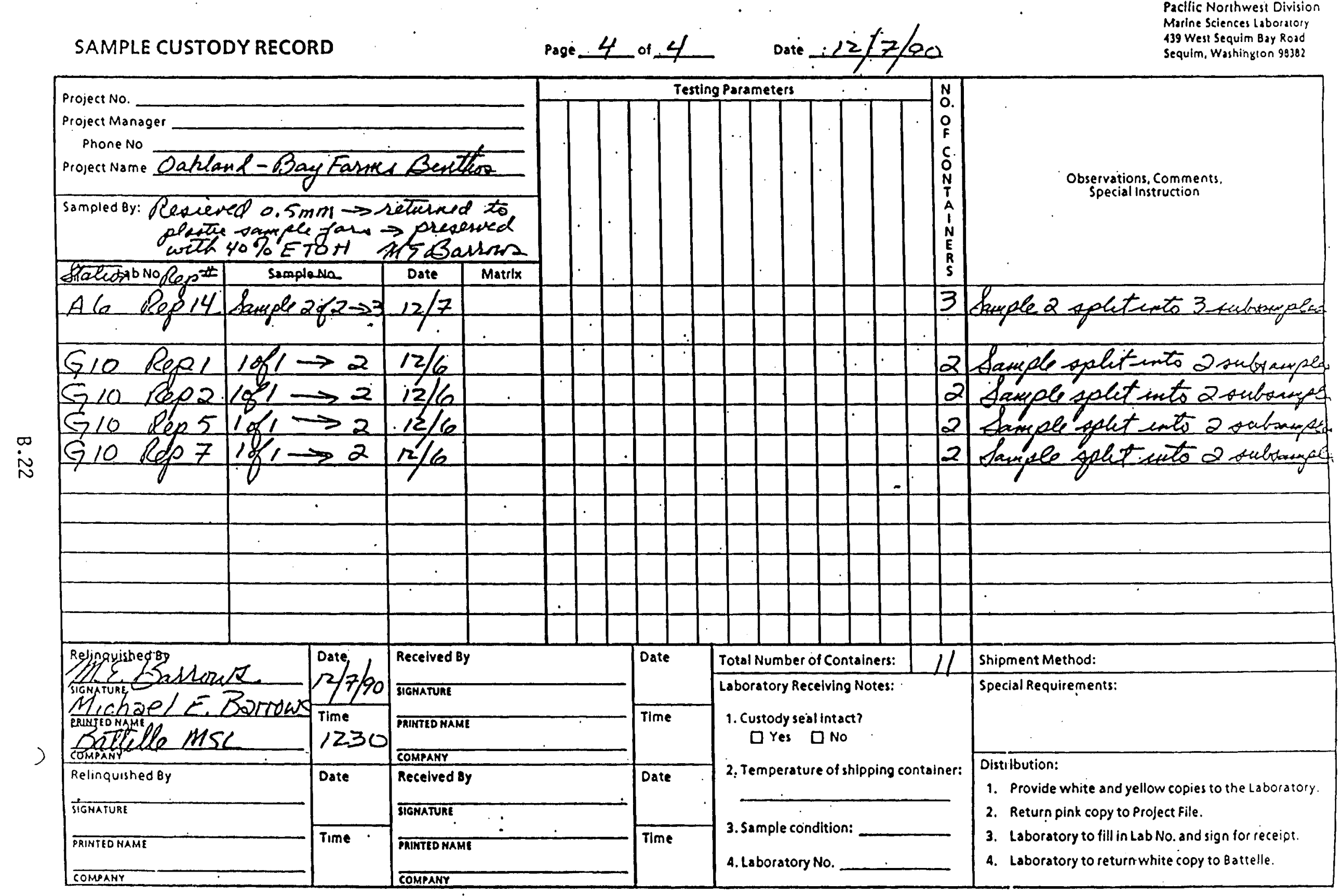




\section{DISTRIBUTION}

No. of

Copies

OFFSITE

12 DOE/Office of Scientific and Technical Information

10 Thomas J. Chase

Civil Engineer

Environmental Branch

U.S. Army Corps of Engineers

San Francisco District

211 Main Street

San Francisco, CA 94105-1905

3 Gene Revelas

Science Application International Corporation 18706 North Creek Parkway

Suite 110

Bothe11, WA 98011
No. of

Copies

ONSITE

DOE Field Office, Richland

P.W. Kruger

23 Pacific Northwest Laboratory

R. Cuello

R.M. Ecker

M.R. Finney

L.F. Lefkovitz

G.P. O'Connor

J.A. Trelstad (2)

J.Q. Word (10)

Publ ishing Coordination

Technical Report File (5) 